\title{
Age Differences in Risk Attitude Are Shaped by Option Complexity
}

\author{
Veronika Zilker, Ralph Hertwig, and Thorsten Pachur \\ Max Planck Institute for Human Development, Berlin, Germany
}

\begin{abstract}
The canonical conclusion from research on age differences in risky choice is that older adults are more risk averse than younger adults, at least in choices involving gains. Most of the evidence for this conclusion derives from studies that used a specific type of choice problem: choices between a safe and a risky option. However, safe and risky options differ not only in the degree of risk but also in the amount of information to be processed-that is, in their complexity. In both an online and a lab experiment, we demonstrate that differences in option complexity can be a key driver of age differences in risk attitude. When the complexity of the safe option is increased, older adults no longer seem more risk averse than younger adults (in gains). Using computational modeling, we test mechanisms that potentially underlie the effect of option complexity. The results show that participants are not simply averse to complexity, and that increasing the complexity of safe options does more than simply make responses more noisy. Rather, differences in option complexity affect the processing of attribute information: whereas the availability of a simple safe option is associated with the distortion of probability weighting and lower outcome sensitivity, these effects are attenuated when both options are more similar in complexity. We also dissociate these effects of option complexity from an effect of certainty. Our findings may also have implications for age differences in other decision phenomena (e.g., framing effect, loss aversion, immediacy effect).
\end{abstract}

Keywords: risky choice, complexity, certainty, cumulative prospect theory, aging

Supplemental materials: http://dx.doi.org/10.1037/xge0000741.supp

In many-perhaps most—of life's decisions, people cannot be certain about which of an option's potential outcomes will actually materialize. At best, they have some information about the probability that the outcomes will occur; this situation is known as decisions under risk (Knight, 1921). A key behavioral regularity in decisions under risk is that people seem to be risk averse: They find riskier options (options with a larger variance in possible outcomes; Markowitz, 1952) less attractive than less risky ones.

This article was published Online First February 6, 2020.

(D) Veronika Zilker, Ralph Hertwig, and Thorsten Pachur, Center for Adaptive Rationality, Max Planck Institute for Human Development, Berlin, Germany.

Results reported in this article were presented at the 59th Conference of Experimental Psychologists, Dresden, Germany, the 60th Conference of Experimental Psychologists, Marburg, Germany, and the 59th Annual Meeting of the Psychonomics Society, Long Beach, California. Veronika Zilker and Thorsten Pachur were involved in study conceptualization and design and writing of the major revisions; Veronika Zilker was involved in experimental software and stimulus design, data collection and curation, data analysis and computational modeling, and writing of the original draft; Veronika Zilker, Thorsten Pachur, and Ralph Hertwig were involved in writing of the final revisions; and Thorsten Pachur was involved in supervision. Data from both studies and code to implement all analyses are hosted at https://osf.io/nzwx4/.

Correspondence concerning this article should be addressed to Veronika Zilker, Center for Adaptive Rationality, Max Planck Institute for Human Development, Lentzeallee 94, 14195 Berlin, Germany. E-mail: zilker@mpib-berlin.mpg.de
To illustrate, when asked to choose between a risky option offering an $80 \%$ chance to win $\$ 4,000$ (otherwise nothing) and a safe option of $\$ 3,000$ guaranteed, most people prefer the latter, although the former option's expected value is higher (e.g., Kahneman \& Tversky, 1979). ${ }^{1}$ Risk attitude-the degree to which people are risk averse or risk seeking - has been shown to be sensitive to a number of factors, such as the domain (e.g., people tend to be risk seeking when evaluating options with possible losses; Kahneman $\&$ Tversky, 1979) and the magnitude of the outcome offered (e.g., people tend to be more risk averse when the outcomes are very high; Holt \& Laury, 2002). In addition, there are considerable individual differences in risk attitude, which have been associated with, for instance, personality (e.g., Becker, Deckers, Dohmen, Falk, \& Kosse, 2012) or cognitive ability (Dohmen, Falk, Huffman, \& Sunde, 2018; Henninger, Madden, \& Huettel, 2010). Moreover, there are robust gender differences, with females often showing higher risk aversion than males (e.g., Charness \& Gneezy, 2012).

Another characteristic that has attracted much attention is age - in particular, how does risky choice differ in older adults relative to younger ones? A common conclusion is that older adults are more risk averse than younger adults in the domain of gains (Best \& Charness, 2015; Mather et al., 2012; Rutledge et al., 2016; Tymula, Rosenberg Belmaker, Ruderman, Glimcher, \& Levy, 2013). In this article, we highlight that much of the evidence

\footnotetext{
${ }^{1}$ The expected value of a risky lottery is defined as the sum of all possible outcomes weighted by their probabilities.
} 
for greater risk aversion in older age stems from one type of choice problem: choices between a safe and a risky option. For instance, when asked to choose between a risky option offering a $20 \%$ chance to win $\$ 50$ (otherwise nothing) and a safe gain of $\$ 10$, older adults are more likely than younger adults to prefer the safe option (e.g., Mather et al., 2012). Choice problems consisting of a safe and a risky option have several practical advantages. For instance, they allow researchers to easily vary the difference in risk between the options by keeping the safe option constant while increasing or decreasing the variance of the risky option, thus capturing finegrained degrees of risk aversion. Safe and risky options, however, differ not only in their degree of risk, but also in the amount of information to be processed-that is, in their complexity. Unlike safe options, risky options consist of multiple pieces of information: Even the simplest risky option consists of two outcomes and their respective probabilities, whereas a safe option is fully described by a single number (the only possible outcome).

We provide evidence that this difference in structural option complexity - defined here as the number of elements that characterize an option-is a key driver of typically observed differences between younger and older adults in risky choice. ${ }^{2}$ We demonstrate that once complexity differences between options are attenuated, age differences in risk attitude disappear. Differences in option complexity between risky and safe options might help to explain puzzling inconsistencies in the literature on age differences in decision making under risk. Last but not least, we investigate the cognitive mechanisms underlying the effect of option complexity on risky choice.

In the following, we first review the evidence regarding age differences in risk attitude and describe the potential role of option complexity in their emergence, and then derive hypotheses about the cognitive mechanisms that might underlie the effects of complexity in risky choice in older and younger adults. Finally, we report an online (Study 1) and a lab study (Study 2) that test these hypotheses by analyzing behavioral patterns and employing computational modeling based on cumulative prospect theory (CPT; Tversky \& Kahneman, 1992).

\section{Age Differences in Risky Choice: An Overlooked Task Dependency}

A standard behavioral approach to examining age differences in risk preferences is to have people make choices between options with differing levels of risk. In most studies with this approach, older adults appear to be more risk averse than younger adults, at least in the domain of gains (e.g., Mather et al., 2012; Rutledge et al., 2016; Tymula et al., 2013). In their meta-analysis summarizing 18 studies using behavioral tasks to examine age differences in risky choice, Best and Charness (2015) concluded that, overall, older adults were more risk averse than younger adults in the gain domain (Hedge's $g=-0.25,95 \%$ confidence interval $[\mathrm{CI}][0.33$, $0.18]$ ), whereas there were no robust age differences in the loss domain $(g=-0.02,95 \%$ CI $[-0.10,0.06])$. Yet findings from some individual studies in the domain of gains violate this pattern. For instance, Mather et al. (2012) did not find general age differences in the tendency to choose the riskier gain (we discuss these results in more detail below), and in Pachur, Mata, and Hertwig's (2017) as well as in Kellen, Mata, and Davis-Stober's (2017) studies, older adults made more risk-seeking choices in the domain of gains than younger adults. Table 1 provides an overview of existing findings (focusing on studies with described probability and outcome information; for an overview of studies in which this information has to be learned from experience, see Mata, Josef, Samanez-Larkin, \& Hertwig, 2011).

Can these seemingly inconsistent results be reconciled? A closer look at the stimuli used in the different studies reveals a striking yet hitherto largely neglected difference. Almost all studies observing higher risk aversion in older than in younger adults in the domain of gains examined choices between a safe and a risky option. In contrast, studies reporting no age differences or the opposite pattern examined primarily choices between two (more or less) risky options: In Pachur et al. (2017) and Kellen et al.'s (2017) studies - both of which found that older adults were more likely to choose the riskier gain-most choice problems consisted of two risky options, such as a choice between Option A, offering $\$ 23$ with a chance of $44 \%$ or $\$ 31$ with a chance of $56 \%$, and Option B, offering $\$ 62$ with a chance of $74 \%$ or $\$ 0$ with a chance of $26 \%$. Likewise, Henninger et al. (2010), who also found higher risk seeking in older adults' choices, employed the Cambridge gambling task, in which all gain options involve risk.

Mather et al. (2012) used both choice problems involving a risky and a safe option and choice problems with two risky options. Age differences in risky choice emerged only if a safe option was available, with older adults showing greater risk aversion in the domain of gains and greater risk seeking in the domain of losses than younger adults. In problems with two risky options, by contrast, there were no age differences. Mather et al. (2012) attributed this finding to a stronger certainty effect in older adults. The certainty effect describes a relative overweighting of certainty. For instance, the difference between $100 \%$ and $85 \%$ is weighted more heavily than the difference between $90 \%$ and $75 \%$ (despite being nominally of the same magnitude). The certainty effect is theoretically accommodated by CPT (Tversky \& Kahneman, 1992), a prominent model that describes regularities in risky choice in terms of nonlinear transformations of outcome and probability information. In CPT, the certainty effect is captured by an inverse S-shaped probability-weighting function that transforms objective probabilities into subjective decision weights (for details and a formal definition see the section "Testing the underlying mechanisms: Computational modeling"). The inverse S-shape of the weighting function has been attributed to affective responses: situations triggering fear or hope (i.e., whenever the probability of winning is less than 1) and situations devoid of those emotions (whenever the probability of winning is equal to 1) are treated as categorically different (Lopes, 1987; Rottenstreich \& Hsee, 2001), leading to large jumps in probability weighting at the extreme ends of the probability scale.

\section{Task-Dependent Age Differences in Risky Choice: The Potential Role of Option Complexity}

Here we offer a different, and, in principle, complementary, explanation of why age differences in risky choice, or a lack

\footnotetext{
${ }^{2}$ Although complexity includes other dimensions, we focus here on the number of elements because it is most relevant for conceptualizing differences between risky and safe options in the common risky choice paradigm.
} 


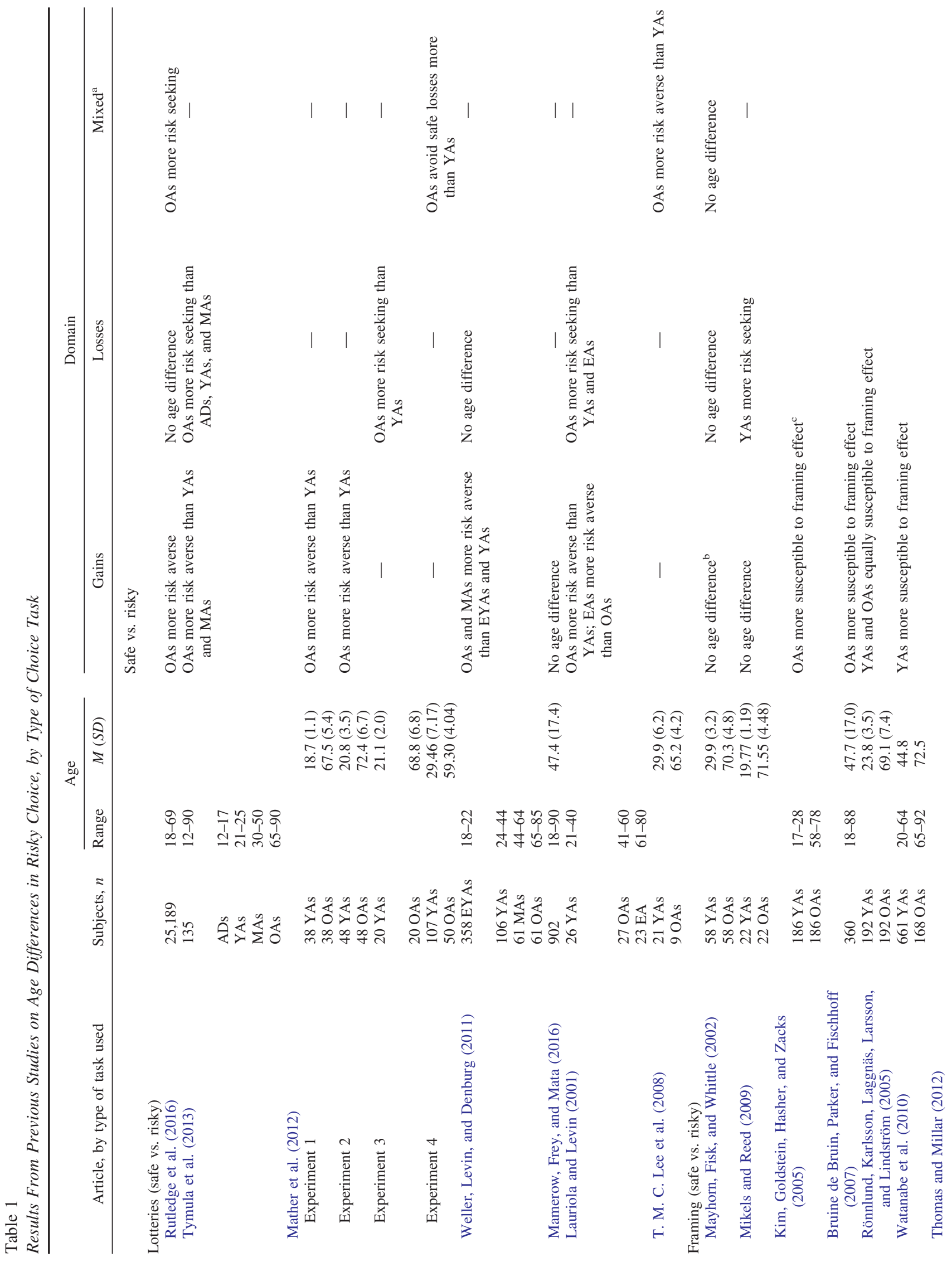




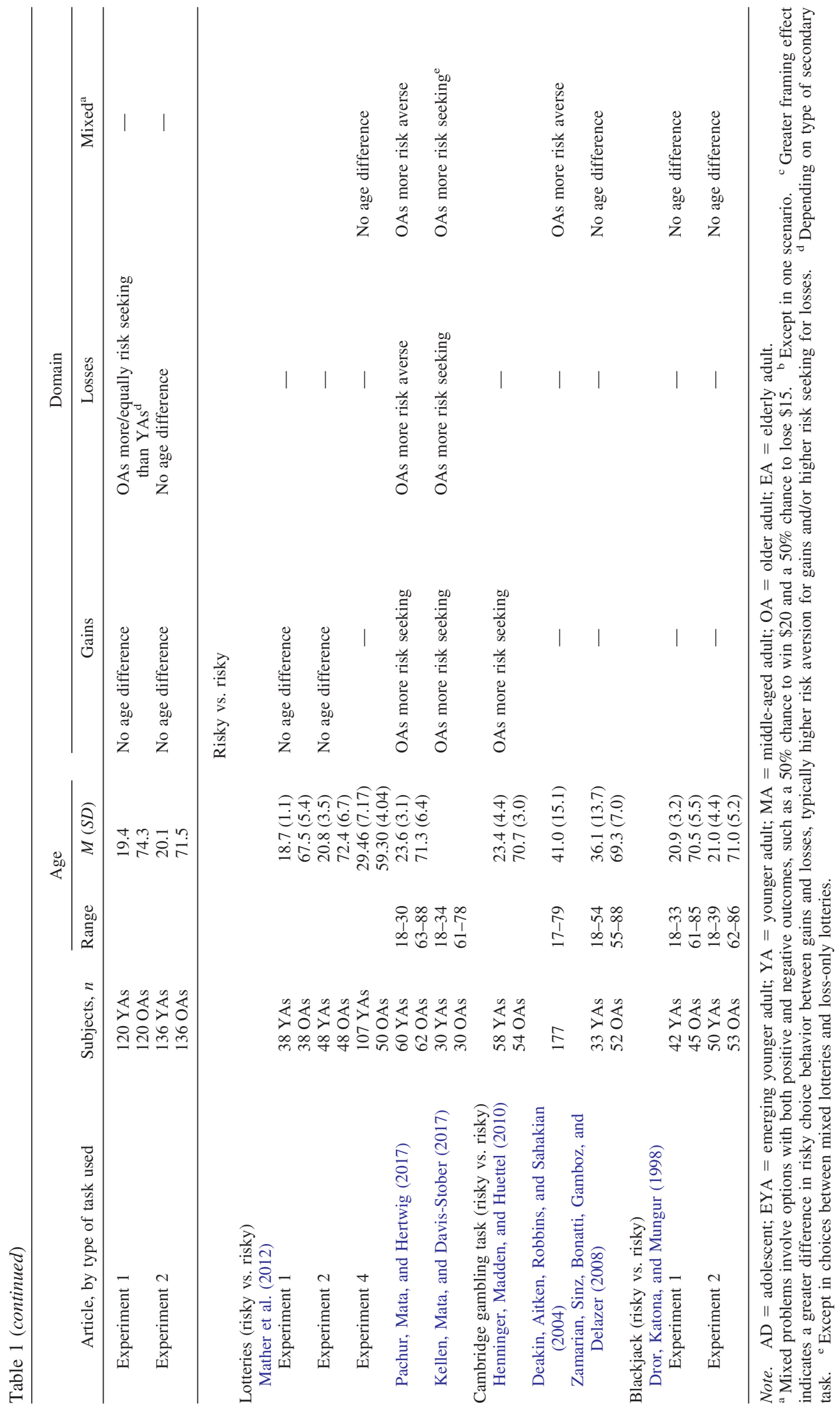


thereof, depend on the presence or absence of a safe option. In contrast to the certainty-effect account, our explanation attributes the differences to cognitive rather than affective factors. It builds on the finding that risk aversion in choices between safe and risky gains is negatively associated with cognitive ability (Dohmen et al., 2018) and the well-documented age-related decline in fluid cognitive ability (Baltes, 1987; Craik \& Bialystok, 2006; Horn \& Cattell, 1967; Salthouse, 2004). Specifically, we argue that the presence of a safe option may influence the emergence (and possibly the direction) of age differences in risk attitude not (or at least, not only) because its outcome is certain, but because a safe option is less complex than a risky option. In choice problems involving a safe and a risky option-in which age differences in risky choice behavior are typically observed-the options differ substantially in complexity. In contrast, in choice problems with two risky options - in which age differences in choice are attenuated, eliminated, or even reversed-differences in complexity between options are much smaller. We suggest that the age differences typically observed in choices involving safe options are not primarily due to genuine differences in risk attitude, or to older adults responding more strongly to certainty than younger adults, but rather stem from older adults' responses to option complexity. This seems plausible given age-related declines in fluid intelligence (Craik \& Bialystok, 2006; Horn \& Cattell, 1967; Zaval, Li, Johnson, \& Weber, 2015), which have been suggested to explain age differences in several dimensions of decision making (e.g., choice, information search), especially in complex and demanding tasks (cf. Frey, Mata, \& Hertwig, 2015; Mamerow, Frey, \& Mata, 2016; Mata, Schooler, \& Rieskamp, 2007; Zaval et al., 2015). On a neurobiological level, these impairments in information processing have been linked to changes in dopaminergic neuromodulation, affecting, for instance, the signal-to-noise ratio of neural processing (Li, Lindenberger, \& Sikström, 2001).
Moreover, aging is associated with structural and functional impairments in the prefrontal cortex (Rypma, Prabhakaran, Desmond, \& Gabrieli, 2001; Salat et al., 2005; West, 1996), which in turn is implicated in decision-relevant working memory functions such as manipulating and integrating different pieces of information (Curtis \& D'Esposito, 2003; D’Esposito et al., 1995; Krawczyk, 2002; Rypma \& D’Esposito, 2000).

Our complexity account and Mather et al.'s (2012) certainty account make divergent predictions about how age differences in risky choice behavior should vary between problem types. Notably, the choice problems used in Mather et al. (2012) do not allow for the possible effects of certainty versus complexity to be disentangled, as the safe options were always less complex than the risky ones. Turning to similarly complex safe and risky options would permit the certainty-effect and the complexity accounts to be dissociated. To construct such a problem type, we increased the complexity of safe options by expressing the safe outcome as a mathematical term rather than a single number, thus rendering its complexity more similar to the complexity of the risky option (see Figure 1 for an example, and the "Materials" section for more detail). Comparing choices in this problem type to choices between a simple safe and a complex risky option isolates the effect of complexity, and comparing them to choices between two complex risky options isolates the effect of certainty. The most basic prediction of the complexity account is that age differences in the tendency to choose the safe option should emerge if the options differ in complexity (involving a simple safe option) but that they should be reduced (or eliminated) with smaller or no differences in option complexity (involving a complex safe option). By contrast, the certainty account does not predict a change in age differences between these two problem types, as both involve a safe option. It does, however, predict reduced age differences in the tendency to choose the less risky option in a condition with two risky options,
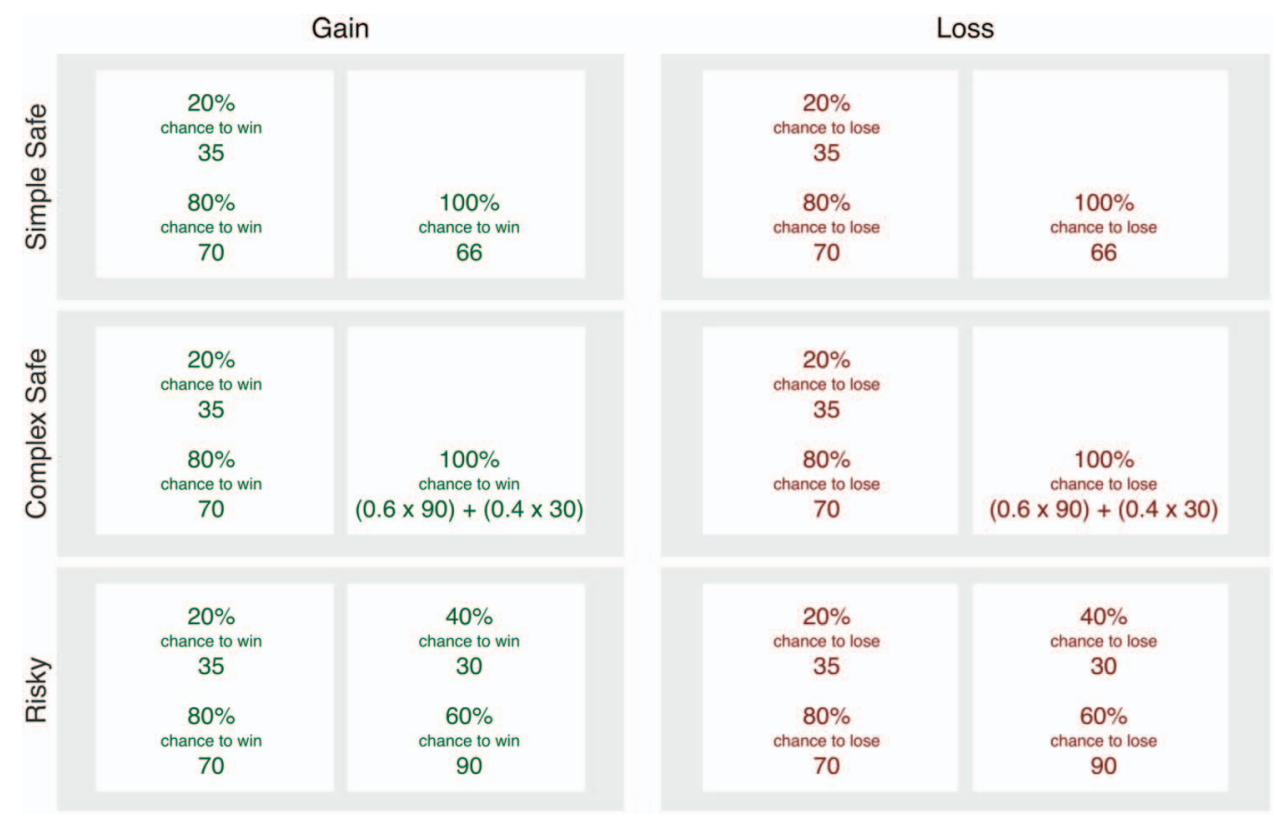

Figure 1. Conditions of the risky choice task: Exemplary choice problems by problem type and domain. See the online article for the color version of this figure. 
compared to a condition with complex safe options-which differ in certainty, but are similar in complexity. Let us emphasize that although they make distinct predictions, the two accounts are not mutually exclusive: Older adults may be more sensitive to both certainty and complexity than are younger adults.

\section{How Might Complexity Affect Age Differences in Risky Choice?}

In addition to examining whether complexity affects the emergence of differences between younger and older adults in risky choice, we were also interested in how complexity might exert its influence on choice behavior. We next describe four candidate mechanisms. Each mechanism entails specific testable predictions, all of which are summarized in Table 2 and elaborated below.

\section{Complexity-Aversion Hypothesis}

One possible mechanism by which option complexity impacts choice behavior is that people find more complex options generally less attractive due to the greater computational effort required to evaluate them (e.g., due to their lower processing fluency-less fluent stimuli are often perceived as less attractive than fluent ones; Alter \& Oppenheimer, 2009). Consistent with this notion of complexity aversion, Bernheim and Sprenger (2019) argued that people prefer lotteries with fewer outcomes that are easier to understand, and that the certainty effect may be a special case of this more general phenomenon. Moreover, both Huck and Weizsäcker (1999) and Sonsino, Benzion, and Mador (2002) found that participants choosing between lotteries that differed in the number of possible outcomes preferred the lottery with fewer outcomes (which were thus less complex). Similarly, in Mador, Sonsino, and Benzion's (2000) study, participants assigned lower prices to more complex lotteries (in terms of the number of outcomes) than to simpler lotteries, even when the simpler lottery had a lower expected value than, or was stochastically dominated by, the more complex lottery. Kováŕík, Levin, and Wang (2016) had their participants rank, in order of preference, lotteries composed of more or less complex sequences of probabilistic events. For instance, a multistage lottery could consist of a coin toss that determined the composition of an urn, with the color of a chip drawn from that urn determining the final outcome. Most participants preferred the simpler but otherwise identical versions to the more complex versions. Due to their declining fluid cognitive abilities, older adults may show a stronger aversion to complexity than younger adults. The complexity-aversion hypothesis predicts that older adults are more averse to more complex options than are younger adults. As a consequence, increasing an option's complexity should decrease older adults' likelihood of choosing that option more than it decreases the likelihood of younger adults choosing it, in both gain and loss domains alike.

\section{Response-Noise Hypothesis}

A second possibility is that rather than directly affecting the subjective attractiveness of the options, complexity increases the error in mapping the valuation of the options onto a response. Response noise is often formalized in the context of a probabilistic choice rule, using a parameter that governs the probability that an option, viewed as more attractive, is actually chosen (e.g., Olschewski, Rieskamp, \& Scheibehenne, 2018; Rieskamp, 2008). In choices between risky lotteries, response noise has been found to be higher under greater cognitive load (Olschewski et al., 2018). To the extent that higher complexity induces cognitive load, it might also increase response noise. Overall, this should shift the proportion of choices of the safe option toward 50\% (risk neutrality). Given that people are typically risk averse in the domain of gains and risk seeking in the domain of losses (Kahneman \& Tversky, 1979), higher response noise should lead to a reduction in risk aversion in the gain domain, and an increase in risk aversion in the loss domain. Since older adults display higher response noise and make more inconsistent choices than younger and middle-aged adults (Pachur et al., 2017; Tymula et al., 2013), the response-noise hypothesis predicts that the increase in response noise under higher complexity will be more pronounced in older than in younger adults. If this is the case, the common age differences in choices between simple safe and complex risky options - that is, older adults making more risk-averse (risk-seeking) choices than younger adults in choices about gains (losses) — should be reduced when both options are similarly complex.

A third possibility is that option complexity affects how people process specific attribute information. That is, rather than generally decreasing an option's attractiveness (as assumed by the complexity-aversion hypothesis) or making mapping of the valuation onto the response more error-prone (as assumed by the response-noise hypothesis), higher complexity might influence how people extract and integrate attribute information on the

Table 2

Possible Mechanisms Underlying an Effect of Option Complexity on Risky Choice and Their Specific Predictions About the Effect of Increasing Safe Options' Complexity on One or Several Outcome Variables

\begin{tabular}{|c|c|c|}
\hline Mechanism & Outcome variable & Prediction \\
\hline Complexity-aversion hypothesis & Risky choice behavior & Increased risk seeking in both gain and loss domain \\
\hline \multirow[t]{2}{*}{ Response-noise hypothesis } & $\rho$ parameter & Decrease in $\rho$ (more noise) \\
\hline & Risky choice behavior & Higher risk neutrality (choice proportion closer to $50 \%$ ) \\
\hline \multirow[t]{2}{*}{ Probability-weighting hypothesis } & $\gamma$ parameter & Increase in $\gamma$ (more linear probability weighting) \\
\hline & Risky choice behavior & $\begin{array}{l}\text { Increased risk seeking in gain domain } \\
\text { Increased risk aversion in loss domain }\end{array}$ \\
\hline \multirow[t]{3}{*}{ Outcome-sensitivity hypothesis } & $\alpha$ parameter & Increase in $\alpha$ (higher outcome sensitivity) \\
\hline & Risky choice behavior & Increased risk seeking in gain domain \\
\hline & & Increased risk aversion in loss domain \\
\hline
\end{tabular}


options. We next describe two hypotheses focused on the processing of attribute information.

\section{Probability-Weighting Hypothesis}

According to the probability-weighting hypothesis, complexity differences affect probability weighting, a key construct in CPT that describes how objective probabilities are transformed into subjective decision weights (a formal description is provided in the section "Testing the underlying mechanisms: Computational modeling"). This hypothesis is based on Glöckner, Hilbig, Henninger, and Fiedler's (2016) finding that choices between a safe and a risky option-which differ in complexity_give rise to a more curved weighting function than choices between two risky options, that do not differ in complexity. ${ }^{3}$

Whereas Glöckner et al.'s (2016) study involved only younger adults, the findings by Mather et al. (2012) suggest that this effect may be even more pronounced in older adults. To recap, Mather et al. (2012) found no age differences in choices between two risky options, whereas older adults had a higher (lower) tendency to choose the safe gains (losses) in choices between a safe and a risky option. The strongly curved weighting function, observed in choices between a safe and a risky option in younger adults (cf. Glöckner et al., 2016), may therefore be even more strongly curved in older adults. In contrast, in choices between two risky options younger and older adults may both show a moderately curved weighting function. This would imply that probability weighting is more sensitive to the availability of a safe option in older than in younger adults.

Our complexity account thus predicts more linear probability weighting in a condition with complex safe options than in a condition with simple safe options, especially in older adults. The certainty-effect account does not predict these differences in probability weighting; rather, it predicts that, due to differences in certainty, probability weighting will differ between problems with complex safe and risky options and problems with two risky options.

\section{Outcome-Sensitivity Hypothesis}

Complexity might also affect how people process outcome information-that is, how they subjectively represent objective outcomes. In CPT, objective outcomes are transformed into subjective values according to a value function, which exponentiates the outcome magnitude by an outcome sensitivity parameter (a formal description is provided in the section "Testing the underlying mechanisms: Computational modeling"). For values of the outcome sensitivity parameter smaller than 1 (i.e., concave value function for gains), differences between the outcomes' magnitudes are attenuated; for values larger than 1 (i.e., convex value function for gains), differences are amplified. Notably, in choices between safe and risky options, the largest outcome in the choice set is typically offered by the risky option (unless the safe option dominates the risky option) such that the value function tends to amplify or attenuate the subjective value of the risky option more than that of the safe option. As a consequence, a more concave value function entails greater risk aversion in the domain of gains and greater risk seeking in the domain of losses.

Based on these insights, we can use Mather et al.'s (2012) results to derive predictions about the possible effects of option complexity on outcome sensitivity. Older adults' stronger risk aversion (seeking) in choices between a simple safe and a risky gain (loss) could indicate a lower outcome sensitivity relative to younger adults. Conversely, a reduced age difference in choice problems with similarly complex options may indicate more similar levels of outcome sensitivity. The outcome-sensitivity hypothesis predicts an increase in outcome sensitivity in problems with complex safe and risky options relative to problems with a simple safe and a risky option, especially in older adults. Note that under the certainty-effect account, no such difference in outcome sensitivity between these two problems types is expected.

To summarize, we have explored four mechanisms-complexity aversion, response noise, probability weighting, and outcome sensitivity - that might contribute to the effect of option complexity on age differences in risky choice. Each mechanism could affect both age groups, which would be indicated by a main effect of problem type (complex safe) on the respective outcome variable (i.e., model parameters or choice behavior; see Table 2). Importantly, the four hypotheses on how complexity influences age differences in risky choice predict that each mechanism is more pronounced in older than in younger adults, which would be indicated by an interaction between complexity and age group on the respective variable. It is also possible that option complexity affects choices through a combination of several mechanisms (unless their predictions are mutually exclusive). For instance, complexity could affect the processing of both probabilities and outcomes, and neither, one, or both of these mechanisms could be more pronounced in older adults.

\section{Study 1}

We tested the basic hypothesis of an effect of option complexity by experimentally manipulating (within-subjects) the complexity of a safe option. The key question was if this manipulation would reduce differences between younger and older adults in the willingness to choose a safe option over a risky option. We also tested the four hypotheses on potential mechanistic underpinnings of this basic behavioral pattern. Whereas the complexity-aversion hypothesis can be tested based on the observed choice behavior alone, testing the response-noise, probability-weighting, and outcomesensitivity hypotheses requires separating the evaluation of probability and outcome information from the influence of response noise. To this end, we modeled choice data with a hierarchical Bayesian implementation of CPT (described in more detail in the section "Testing the underlying mechanisms: Computational modeling"). The role of CPT as a measurement model and potential underpinnings of its parameters in terms of cognitive processing strategies are addressed in more detail in the General Discussion. Finally, we also examined risky choices without a safe option and without differences in option complexity, by including a condition involving two risky options.

\section{Method}

Participants. The experiment was conducted online, using Prolific Academic to recruit participants. We targeted younger and

\footnotetext{
${ }^{3}$ We refer to the data in the description condition.
} 
older adults based on age range (18-35 years and $\geq 55$ years, respectively) using the Prolific Academic prescreening tool. Only individuals conforming to the specified age ranges were invited to participate. Participants were removed from the sample if they did not complete the survey, or if their age or gender, as identified by the Prolific Academic prescreening tool, diverged from their responses to the demographic questions at the end of the survey. To ensure that participants had read the instructions and understood the task, we asked a simple comprehension question on the same screen frame. ${ }^{4}$ Participants who failed this item were excluded from the sample. The final sample of participants consisted of 82 younger adults and 76 older adults. Demographic characteristics, numeracy scores, and self-reported risk preferences are described in Table 3. Participants who finished the experiment received a basic payment of $£ 4.20$ as well as a performance-contingent monetary bonus. The bonus was determined individually for each participant by randomly selecting one trial and playing out the chosen option. The resulting outcome was converted from the experimental currency E\$ ("E-dollar") into pounds $(\mathrm{E} \$ 100=£ 1)$. Participants were informed about this reward scheme before starting the choice task.

\section{Materials}

Risky choice task. In the main task, participants were presented with 108 two-option choice problems. Each problem consisted of either a safe and a risky option, or two risky options (depending on the condition). Twelve choice problems included a stochastically dominated option, in which all outcomes were lower than all outcomes of the other option. We included these problems to assess data quality. The main analyses of risk attitude reported below include the nondominated problems only.

In the risky choice task, each option offered monetary outcomes, described in terms of the experimental currency E\$, and the probabilities of these outcomes, expressed as percentages. In half of the choice problems, the riskier option (both in terms of variance and in terms of coefficient of variation; Weber, Shafir, \& Blais, 2004) had a higher expected value; in the other half, the less risky option had a higher expected value. The problem set did not involve choices between equal-expected-value options. This is because only problems with unequal expected values allow for measuring decision quality (the proportion of choices of the option with the higher expected value, cf. Pachur et al., 2017). For each problem, participants were asked to indicate which option they preferred and how confident they were in this preference on a 10-point confidence scale ranging from very confidently $A$ to very confidently $B$, where A and B referred to the options Lottery A and

Table 3

Characteristics of the Sample in Study 1 by Age Group

\begin{tabular}{lcc}
\hline \multicolumn{1}{c}{ Characteristic } & Younger & Older \\
\hline Participants, $N$ & 82 & 76 \\
Gender (female), $n$ & 39 & 41 \\
Age (years) & $26(4.2)$ & $60.4(4.4)$ \\
$\quad$ Range (years) & $18-34$ & $55-72$ \\
Self-reported risk preference & $5.9(2.2)$ & $5.4(2.2)$ \\
Numeracy & $2.6(1.2)$ & $2.3(1.2)$ \\
\hline
\end{tabular}

Note. Data are presented as $M(S D)$ unless otherwise indicated.
Lottery B. (In our analyses below, however, we focus on the binary choices.) Screen shots and a timeline for the task can be found in the online supplemental materials.

There were three types of choice problems (see Figure 1). In each type, one option was risky, offering two possible outcomes with some probability (adding up to $100 \%$ ). Depending on problem type, this risky option was paired with either a simple safe option, a complex safe option, or another (less) risky option. In the simple safe condition, the safe option offered one outcome, expressed as a single number, with certainty (100\%). In the complex safe condition, the safe option offered the same certain $(100 \%)$ outcome magnitude as the simple safe condition, but this outcome was expressed as a mathematical term in which two integers had to be multiplied by a number between 0.01 and .99 (rounded to the second digit and adding up to one) and then summed up (see Figure 1). For instance, a safe outcome of E\$66 was expressed as $(0.6 \times 90)+(0.4 \times 30) \mathrm{E} \$$. Finally, in the risky condition, both options were risky, but one was riskier than the other. The second risky option was constructed using the same components as in the mathematical term in the complex safe condition: The two integers were used as the outcomes, and the weights as their probabilities (adding up to 100\%). For example, the complex safe outcome of $(0.6 \times 90)+(0.4 \times 30) \mathrm{E} \$$ corresponded to a risky option offering E\$90 with $60 \%$ and $\mathrm{E} \$ 30$ with $40 \%$. Note that the risky condition and the complex safe condition were similarly complex: In both conditions, calculating each option's objective value required multiplying two sets of numbers and adding up the results. ${ }^{5}$ The construction principle for the choice problems also ensured that expected values and differences in expected values were balanced across all three conditions. The outcomes were randomly sampled from a uniform distribution ranging from 1 to 100 . To prevent participants from recognizing options from a previous choice problem in a different condition, the outcomes of corresponding choice problems were randomly jittered by \pm 2 across the conditions. The first outcome's probability was obtained by randomly sampling from a uniform distribution ranging from 0.01 to 0.99; the second outcome's probability was the difference between the obtained value and 1 . In all three conditions, half of the choice problems involved gain outcomes; the other half, loss outcomes. Choice problems with losses were constructed by reflecting the outcomes of the choice problems with gains into the loss domain. We provide a full list of all 108 choice problems in the online supplemental materials, where we also display choice proportions of younger and older adults on each individual problem.

Every participant made choices in all conditions and both domains. The choice problems were presented in a randomized order that was uniquely determined for each participant. We also randomized - uniquely for each participant - the side of the screen on which the high and low risk options appeared on each choice

\footnotetext{
${ }^{4}$ The item read as follows: "To demonstrate that you have understood the task, please indicate which is the correct option below: (1) All gambles involve losses. (2) All gambles involve gains. (3) The equations shown on some gambles express probabilities. (4) The equations shown on some gambles express outcomes." Response 4 was correct.

5 This manipulation was not meant to make participants adhere to expected-value calculation, but rather aimed to balance the surface features of the options in terms of complexity.
} 
problem. Response times in the risky choice task were recorded in milliseconds.

Complexity rating. In order to measure the extent to which the participants perceived the different types of choice problems as varying in complexity, we asked them to rate, on a 6-point scale ranging from 1 (very low complexity) to 6 (very high complexity), the subjective complexity of a subset of 30 randomly drawn choice problems from the various conditions.

Self-reported risk preference. In order to explore how participants' decisions in the three conditions of the risky choice task related to their self-reported risk preference, we asked them to indicate their risk preference on a one-item general risk question:

How do you see yourself? Are you generally a person who is fully prepared to take risks or do you try to avoid taking risks? Please tick a box on the scale, where the value 0 means not at all willing to take risks and the value 10 means very willing to take risks.

This is a standard item which has been used, for instance, to assess the risk preferences in the German Socio-Economic Panel (see Dohmen et al., 2011) and across age cohorts (Josef et al., 2016).

Berlin Numeracy Test. As the more complex choice problems involved more challenging numerical operations, we explored the role of numerical abilities and measured participants' numeracy, using the adaptive, computerized version of the Berlin Numeracy Test (Cokely, Galesic, Schulz, Ghazal, \& GarciaRetamero, 2012). This adaptive test consists of two to four items (depending on a person's responses) and is normed to divide participants into quartiles based on their numerical skills.

Design. The experiment had a mixed design, with age group as between-subjects factor and type of choice problem (simple safe, complex safe, and risky) and domain (gains vs. losses) as within-subjects factors. The experiment was approved by the institutional review board of the Max Planck Institute for Human Development.

Procedure. The experiment was programmed in the survey software Unipark (QuestBack GmbH, 2016). Participants from the subject pool of Prolific Academic were approached based on age as previously delineated and invited via e-mail. Upon clicking the invitation link, participants were directed to the questionnaire, informed about privacy and data-protection guidelines, and asked for informed consent. Participants who did not provide informed consent were not able to proceed to the study. Next, participants received instructions regarding the risky choice task, its baseline payment, and the incentivization scheme; they then completed this task, the complexity rating, and the numeracy task (in that order). After completing all tasks, participants indicated their gender and age in years and answered the self-report item on risk preference. They also had the opportunity to comment on the study in an open-answer written format. Participants then clicked on a link to get redirected to Prolific Academic and confirm that they had completed the study. Submissions were accepted after the data had been checked against the criteria described above, which resulted in participants receiving the basic payment. The bonus payments were determined after all participants had completed the experiment. If the randomly selected trial for a participant happened to be a loss trial, no bonus was paid out.

\section{Results}

The behavioral analyses were performed in RStudio (Version 1.1.463) running under macOS 10.14.4. Computational modeling was performed on a Windows server in RStudio (Version 1.1.463) and JAGS-4.3.0. All Bayesian generalized linear mixed-effects regression (GLMER) analyses reported below were implemented using the rstanarm package (Goodrich, Gabry, Ali, \& Brilleman, 2018). Individual effects in GLMERs were considered credible if the $95 \%$ posterior interval for the coefficient excluded zero. The posterior intervals, sometimes also referred to as credible intervals, cover the central $95 \%$ of the posterior distribution of the estimated coefficients, and can be interpreted as covering the range that includes the true parameter values with $95 \%$ probability (cf. Morey, Hoekstra, Rouder, Lee, \& Wagenmakers, 2016). All GLMER analyses were conducted separately for the gain and loss domains, given the evidence for domain-specific age differences in risk attitude in the previous literature (Best \& Charness, 2015). When reporting the effects of the factor problem type (which has three levels), the simple safe condition serves as the reference condition unless specified otherwise. In brackets we specify the condition that was compared to the reference condition. For instance, a main effect of problem type (complex safe) refers to the comparison between the simple safe and the complex safe condition-that is, the effect of complexity. An interaction between problem type (complex safe) and age group (older) describes whether the difference between the simple safe and the complex safe condition was more pronounced for older than for younger adults - that is, whether older adults showed a stronger response to complexity. For the factor age group the younger adults served as the reference group.

To first assess the quality of the choice data, we inspected the responses in the risky choice problems including a dominated option. Across all problem types, participants chose the dominating option in $69.22 \%$ of trials in the domain of gains (average choice proportion for younger adults: $73.96 \%$; older adults: $64.19 \%$ ) and in $88.46 \%$ of trials in the domain of losses (younger adults: $88.82 \%$; older adults: $88.07 \%$ ). The high overall rate of choices of the dominating option indicates relatively good data quality. Further analyses of the choices on the problems with a dominated option are reported in Appendix A.

Was the complexity manipulation successful? We used Bayesian GLMERs to analyze participants' complexity ratings of the three problem types. Detailed results are reported in Table A1 and illustrated in Figure A2 in Appendix A. Participants rated the choice problems from the complex safe condition and those from the risky condition as more complex than those from the simple safe condition, indicating that the complexity manipulation was successful.

We also examined the effect of the complexity manipulation on response times in the risky choice task, using Bayesian GLMERs. Detailed results are reported in the bottom panel of Table A1 and illustrated in Figure A3 in Appendix A. Most importantly, and further supporting the conclusion that the complexity manipulation successfully increased the complexity of the problems, participants took longer to make choices in the complex safe condition and the risky condition than in the simple safe condition. Further, older adults took more time for their choices overall than did younger adults. In sum, the analyses show that our manipulation increased, 
as intended, the complexity of the safe options both subjectively (in terms of complexity ratings) and objectively (in terms of the time spent on solving the task).

Did complexity affect age differences in risky choice? Next, we tested the basic hypothesis about the effects of complexity on behavior in the risky choice task, according to which age differences in risky choice should be reduced or even eliminated in choices between more similarly complex options (for an analysis of decision quality, the tendency to choose the option with the higher expected value, see Appendix F). The empirical choice proportions of the less risky option in each problem type, domain, and age group are displayed in the top panel of Figure 2. The observed qualitative patterns support the basic hypothesis that older adults are more sensitive to differences in option complexity than are younger adults: Whereas in the condition with simple safe options older adults appear more risk averse in the domain of gains and more risk seeking in the domain of losses than do younger adults, these age differences are attenuated in the other conditions, where the options are more similar in complexity.

We next evaluated the statistical credibility of these qualitative patterns. According to our basic hypothesis, we expected an interaction between age group and problem type on the tendency to choose the riskier option. To test this hypothesis, we conducted Bayesian mixed-effects logistic regressions using the choice of the riskier option as the dependent variable, and problem type and age group (main effect model) as well as their interaction (interaction model) as fixed effects. The models further included fixed effects for the expected value difference between options, a dummy variable indicating whether the option with the higher expected value was also riskier, each participant's numeracy score, and their self-reported risk preference. The models included a random intercept for each participant. Coefficients and $95 \%$ posterior intervals are displayed in Table 4.

In the gain domain, when both options were similarly complex, the tendency to choose the riskier option increased more in older than in younger adults, as indicated by the credible interaction of problem type (complex safe) and age group (older). This statistically corroborates our basic hypothesis about choice behavior, and the qualitative pattern apparent in Figure 2, for the domain of gains: Older adults are more sensitive to differences in option complexity than younger adults. In the loss domain, the interaction between problem type (complex safe) and age group (older) was not credible.

We also conducted a more liberal test for the main effect of age group on risky choice behavior within each condition, using Bayesian mixed-effect logistic regressions. ${ }^{6}$ Detailed results are reported in Table B1 in Appendix B. To summarize the key findings, in the condition with simple safe options, older adults made credibly more risk-averse choices in the domain of gains, and credibly more risk-seeking choices in the domain of losses, compared to younger adults. No credible differences between younger and older adults emerged in the conditions with similarly complex safe and with risky options in both domains. That is, although in the domain of losses the interaction between problem type (complex safe) and age group was not credible in the model with the full data, the analysis of main effects in the individual conditions extends the support for our basic hypothesis to the domain of losses: Age differences in risky choice were eliminated when both options were similarly complex, in both domains.
Having established the behavioral effect of complexity on age differences in risky choice, we next tested the four hypotheses regarding the potential underlying mechanisms of this effect (see Table 2).

Testing the underlying mechanisms: Complexity aversion. The first of our four hypotheses, the complexity-aversion hypothesis, can be tested based on choice patterns alone. To recap, according to this hypothesis, increasing an option's complexity should make it less attractive, both in the gain and loss domains. To test this hypothesis, we evaluated the direction of the effect of the complexity manipulation on risky choice behavior within each age group, using Bayesian logistic mixed-effect regressions. Detailed results are reported in Table B3 in Appendix B.

Increasing the complexity of safe options made older adults less likely to choose the safe options in the domain of gains, but not in the domain of losses: There was a slight but noncredible trend indicating that increasing the complexity of safe losses made older adults more likely to choose these safe options. ${ }^{7}$ That is, whereas older adults found safe gains less attractive when their complexity increased, they found safe losses equally or even more attractive when their complexity increased. This result from the domain of losses allows us to discard the complexity-aversion hypothesis, which predicts that increasing an option's complexity should make it less attractive, irrespective of outcome domain. We conclude that the higher sensitivity to option complexity of older compared to younger adults is not simply due to more aversion to complexity.

Next, we turn to the remaining three candidate mechanisms that may underlie the effect of complexity on age differences in risky choice: response noise, probability weighting, and outcome sensitivity (see Table 2). We used computational modeling with CPT to evaluate these hypotheses.

Testing the underlying mechanisms: Computational modeling. We modeled participants' choices with a hierarchical Bayesian implementation of CPT (see also Nilsson, Rieskamp, \& Wagenmakers, 2011; Scheibehenne \& Pachur, 2015). In CPT, each option's objective outcomes $x_{i}$ are transformed into subjective values according to the value function $v$ :

$$
v\left(x_{i}\right)=\left\{\begin{array}{cl}
x_{i}^{\alpha^{\text {gain }}}, & \text { if } x_{i} \geq 0 \\
-\left(\left|x_{i}\right|\right)^{\alpha^{l o s s}}, & \text { if } x_{i}<0
\end{array},\right.
$$

with $\alpha \epsilon[0,2]$. The outcome sensitivity parameter $\alpha$ modulates the curvature of the value function and captures the sensitivity to differences in outcomes. A parameter value of $\alpha=1$ indicates linear (objective) treatment of outcomes and thus high outcome sensitivity. Values of $\alpha<1$ indicate a concave (convex) value function for gains (losses) and diminishing sensitivity to outcomes; values of $\alpha>1$ indicate a convex (concave) value function for gains (losses). Note that because our choice problems did not include mixed lotteries, the model's value function does not have a loss aversion parameter.

\footnotetext{
${ }^{6}$ This test can be considered more liberal since main effects can be identified with higher power than interactions given the same sample size.

${ }^{7}$ In younger adults, increasing the complexity of safe options decreased the tendency to choose these safe options in the domain of gains, but this effect was weaker than in older adults. Younger adults' choices were not credibly affected by complexity in the domain of losses.
} 

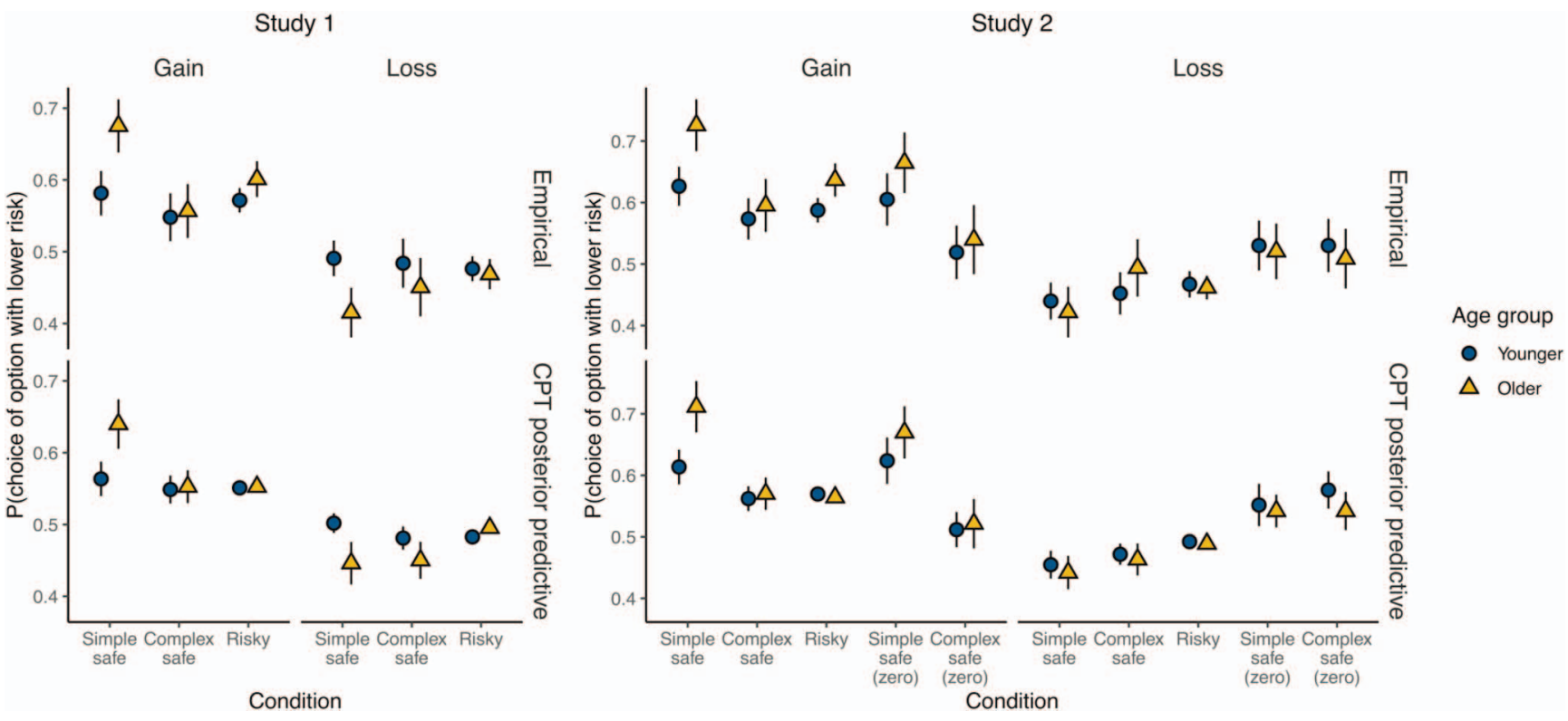

Figure 2. Empirical and posterior predictive- that is, predicted by cumulative prospect theory (CPT) based on the estimated parameters - choice proportions for the nondominated problems in all conditions and age groups by domain. Error bars indicate $95 \%$ confidence intervals. Age differences in the tendency to choose the low risk option are more pronounced in the simple safe problem type, where the options differ considerably in complexity, than in problems involving more similarly complex options. See the online article for the color version of this figure.

Further, decision weights $\pi$ for each outcome are determined based on transforming cumulative objective probabilities $p$ using the probability-weighting function $\omega$ :

$$
\omega\left(p_{i}\right)=\frac{p_{i}^{\gamma}}{\left[p_{i}^{\gamma}+\left(1-p_{i}^{\gamma}\right)\right]^{1 / \gamma}},
$$

with $\gamma \in[0,2]$. For a detailed description of how cumulative weights $\pi$ are derived from $\omega$, see Tversky and Kahneman (1992). The parameter $\gamma$ governs the shape of the probability-weighting function and reflects the degree of nonlinear distortion of objective probabilities. The probability-weighting function is linear under $\gamma=1$. Values of $\gamma<1$ entail an inverse S-shaped probabilityweighting function; values of $\gamma>1$ entail an S-shaped probabilityweighting function. An inverse S-shaped probability-weighting function indicates reduced sensitivity to differences in probabilities in the middle range and a relative amplification of extreme probabilities - thus accommodating the certainty effect. An S-shaped probability-weighting function, in contrast, indicates reduced sensitivity at the extreme ends of the probability scale and a relative amplification of differences in probabilities in the middle range of the scale.

The overall valuation $V$ of each option is then determined by multiplying the subjective values of its outcomes by the corresponding decision weights, and then summing up across the outcomes within each option:

$$
V=\sum \pi_{i} \cdot v\left(x_{i}\right) .
$$

Choice probabilities are then derived from the valuations of options A and B using the logit choice rule (cf. Stott, 2006), which defines the probability that option A is chosen over option B as

$$
p(A, B)=\frac{1}{1+e^{\rho[V(B)-V(A)]}} .
$$

The response noise parameter $\rho>0$ captures the extent to which choices deterministically follow the difference in valuation between the options. With $\rho=0$ the choice probability is 0.5 (i.e., choice behavior is random and not a function of the valuations of the options). With increasing values of $\rho$, the probability of choosing the option with the higher valuation approaches 1 . As the effects of complexity on choice varied across domains, we defined $\alpha, \gamma$ and $\rho$ separately for the gain and loss domains. This model structure allowed us to examine differential effects of complexity on CPT's parameters between the domains.

In Bayesian parameter estimation, parameters are initially represented in terms of prior distributions and then updated into posterior distributions in the light of the data. In the hierarchical approach, model parameters are estimated for each participant individually and the individual-level parameters are assumed to be drawn from a group-level distribution. This approach acknowledges dependencies between data points due to common sources of variation (M. D. Lee, 2011; Nilsson et al., 2011). We estimated the individual-level and group-level posterior distributions for all parameters, separately for younger and older adults, and for the different conditions of the complexity manipulation in both studies. The CPT model was implemented in JAGS-4.3.0 and estimated using the jags.parallel function from the R2jags package (Su \& Yajima, 2015). We ran 30 parallel chains of 101,000 samples each, each including an initial burn-in period of 1,000 samples that were discarded from analysis (cf. Kruschke, 2014). To reduce autocorrelation, the chains were thinned such that every 20th sample was recorded. We assessed convergence via the 


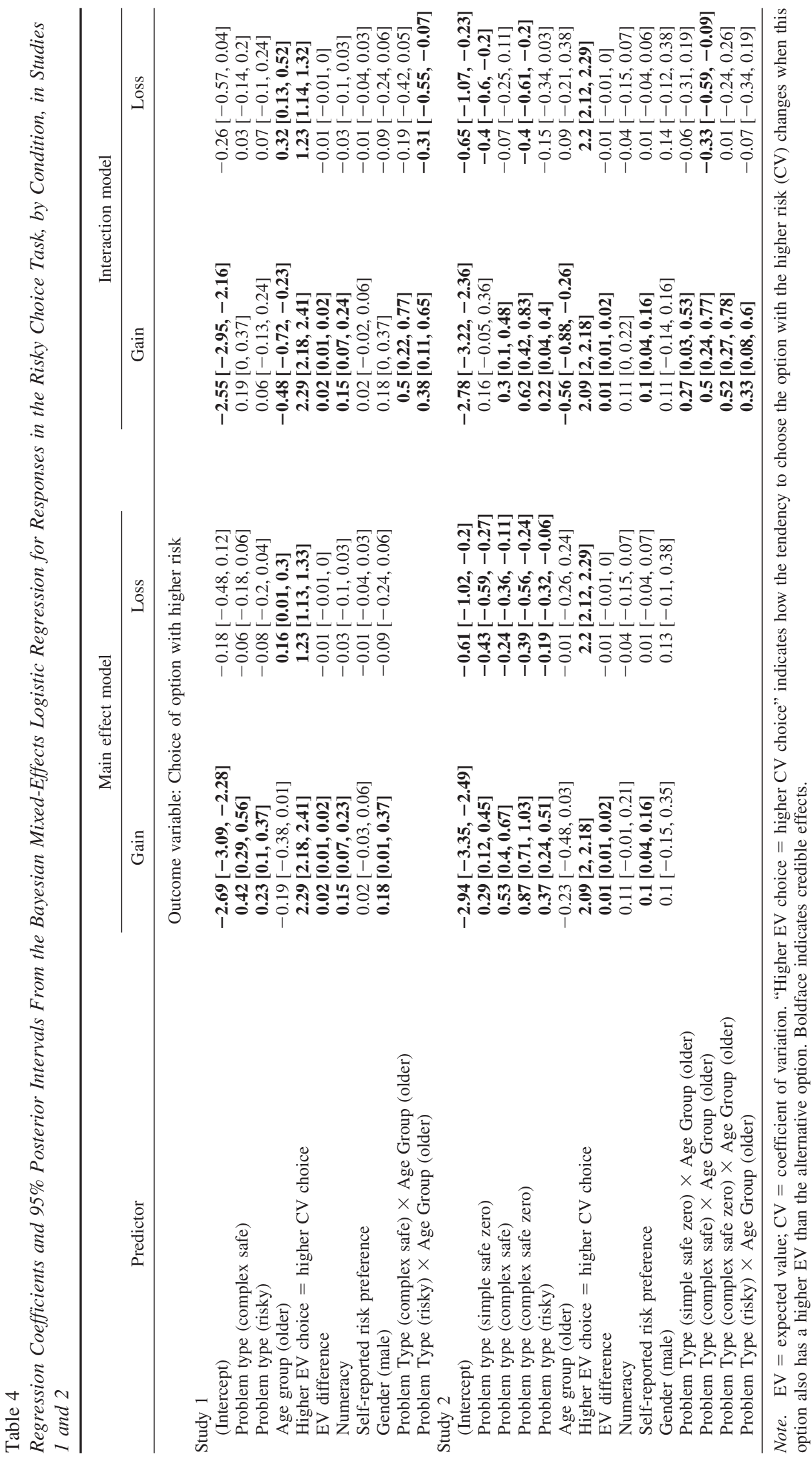


potential scale reduction factor $\hat{R}$ (Gelman \& Rubin, 1992), which was smaller than 1.03 for all estimated parameters, indicating good convergence. To assess whether our computational modeling approach could disentangle the various components of CPT we also conducted an extensive parameter recovery analysis. The analysis demonstrated good recoverability of the parameters and is reported in the online supplemental materials.

We assessed the degree to which the estimated CPT model captured the empirical choice patterns by inspecting the posterior predictive choice probabilities based on the posterior estimates of the CPT parameters for each condition, domain, and participant. The results for risk attitude (i.e., the tendency to choose the less risky option) are shown in Figure 2. As can be seen, the posterior predictive choice probabilities reproduced the qualitative patterns in the empirical data well. Based on the posterior estimates, CPT predicts age differences in choice probabilities in the simple safe condition, which are eliminated in the complex safe and the risky condition. This shows that the effects of option complexity on age differences in risky choice can be accounted for by CPT as a whole. The question remains which specific construct(s) of CPT are affected by complexity-that is, whether complexity acts via the mechanism described by the response-noise hypothesis, the probability-weighting hypothesis, the outcome-sensitivity hypothesis, or a combination thereof (see Table 2). To test this, we conducted a series of Bayesian generalized linear model (GLM) analyses comparing the individual-level parameter estimates of CPT across the different conditions and age groups. Each hypothesis predicts effects of problem type on the respective parameter of the CPT analysis $(\rho, \gamma$, and $\alpha)$ in both domains (for a summary of the predictions see Table 2). In separate Bayesian GLMs, we first analyzed the effects of age group and problem type on the means of the individual-level posterior distributions of each parameter (main effect models). To further test whether older adults were more sensitive to the complexity manipulation than younger adults on any parameter, we estimated a second set of models that also included the interaction between age group and problem type (interaction models). For the models reported in the main text, we used the condition with simple safe options as the reference condition for the problem type factor. Comparing the problem type (complex safe) with this reference allowed us to evaluate the effects of complexity on the model parameters predicted by the response-noise hypothesis, the probability-weighting hypothesis, and the outcome-sensitivity hypothesis.

Response-noise hypothesis. According to this hypothesis, complexity increases response noise, and this effect is more pronounced in older than in younger adults. We tested this hypothesis using Bayesian GLMs with individual-level estimates of the response noise parameter $\rho$ as the dependent variable and the simple safe condition as the reference condition for the effect of problem type. GLM results are displayed in Table 5. Figure 3 shows the means and $95 \%$ CIs of the estimated individual-level posterior means for $\rho$, for each age group and domain. In both gains and losses, there was a negative main effect of age group, which indicates that $\rho$ was lower-and response noise thus higher-in older than in younger adults. In both domains, there was also a negative main effect of problem type (complex safe), meaning that for both age groups response noise was higher in the complex safe than in the simple safe condition. Next, we evaluated the interac-

Table 5

Regression Coefficients and 95\% Posterior Intervals for the Generalized Linear Models Predicting Parameters of the Cumulative Prospect Theory Analysis in Study 1

\begin{tabular}{|c|c|c|c|c|}
\hline \multirow[b]{2}{*}{ Predictor } & \multicolumn{2}{|c|}{ Gain } & \multicolumn{2}{|c|}{ Loss } \\
\hline & Main effect model & Interaction model & Main effect model & Interaction model \\
\hline \multicolumn{5}{|c|}{ Outcome variable: $\rho$ (response noise) } \\
\hline (Intercept) & $0.2[0.19,0.21]$ & $0.2[0.19,0.22]$ & $0.27[0.25,0.29]$ & $0.28[0.26,0.31]$ \\
\hline Age group (older) & $-0.03[-0.04,-0.02]$ & $0.04[0.06,0.02]$ & $-0.07[-0.09,-0.05]$ & $-0.09[-0.13,-0.06]$ \\
\hline Problem type (complex safe) & $-0.1[-0.11,-0.09]$ & $-0.12[-0.13,-0.1]$ & $-0.09[-0.12,-0.07]$ & $-0.12[-0.16,-0.09]$ \\
\hline Problem Type (complex safe) $\times$ Age Group (older) & & $0.04[0.01,0.06]$ & & $0.06[0.01,0.11]$ \\
\hline Problem type (risky) & $-0.03[-0.05,-0.02]$ & $-0.03[-0.05,-0.01]$ & $-0.08[-0.11,-0.06]$ & $-0.09[-0.13,-0.06]$ \\
\hline Problem Type (risky) $\times$ Age Group (older) & & $-0.01[-0.03,0.02]$ & & $0.02[-0.03,0.07]$ \\
\hline \multicolumn{5}{|c|}{ Outcome variable: $\gamma$ (probability weighting) } \\
\hline (Intercept) & $0.77[0.72,0.82]$ & $0.71[0.64,0.77]$ & $0.78[0.74,0.82]$ & $0.84[0.79,0.89]$ \\
\hline Age group (older) & $-0.06[-0.11,0]$ & $0.08[-0.01,0.17]$ & $-0.02[-0.06,0.02]$ & $-0.16[-0.22,-0.09]$ \\
\hline Problem type (complex safe) & $0.44[0.37,0.5]$ & $0.51[0.43,0.6]$ & $0.39[0.34,0.44]$ & $0.23[0.17,0.3]$ \\
\hline Problem Type $($ complex safe $) \times$ Age Group (older) & & $-0.16[-0.28,-0.03]$ & & $0.33[0.23,0.43]$ \\
\hline Problem type (risky) & $0.61[0.55,0.68]$ & $0.73[0.64,0.82]$ & $0.49[0.44,0.55]$ & $0.46[0.4,0.53]$ \\
\hline Problem Type (risky) $\times$ Age Group (older) & & $-0.25[-0.38,-0.12]$ & & $0.07[-0.03,0.16]$ \\
\hline \multicolumn{5}{|c|}{ Outcome variable: $\alpha$ (outcome sensitivity) } \\
\hline (Intercept) & $0.72[0.66,0.77]$ & $0.81[0.75,0.87]$ & $1.17[1.11,1.23]$ & $1.26[1.18,1.33]$ \\
\hline Age group (older) & $-0.1[-0.15,-0.04]$ & $-0.29[-0.38,-0.2]$ & $0.02[-0.05,0.08]$ & $-0.17[-0.28,-0.06]$ \\
\hline Problem type (complex safe) & $0.24[0.18,0.31]$ & $0.12[0.03,0.21]$ & $-0.06[-0.14,0.02]$ & $-0.12[-0.23,-0.01]$ \\
\hline Problem Type (complex safe) $\times$ Age Group (older) & & $0.25[0.12,0.38]$ & & $0.13[-0.03,0.29]$ \\
\hline Problem type (risky) & $-0.32[-0.39,0-.26]$ & $-0.49[-058,-0.39]$ & $-0.46[-0.54,0.38]$ & $-0.66[-0.77,-0.56]$ \\
\hline Problem Type (risky) $\times$ Age Group (older) & & $0.35[0.22,0.48]$ & & $0.42[0.27,0.57]$ \\
\hline
\end{tabular}

Note. Boldface indicates credible effects 

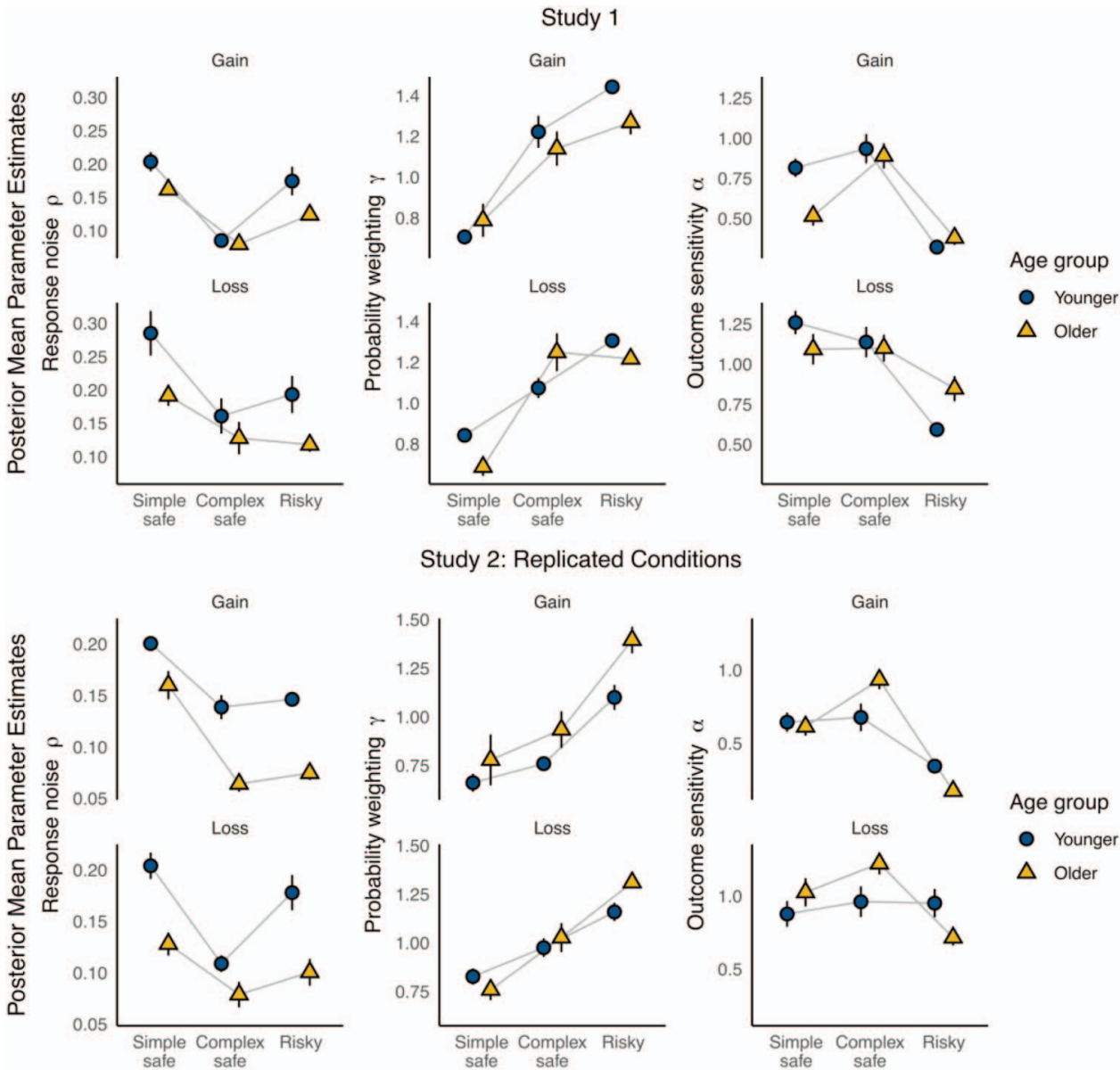

Age group

- Younger

$\Delta$ Older
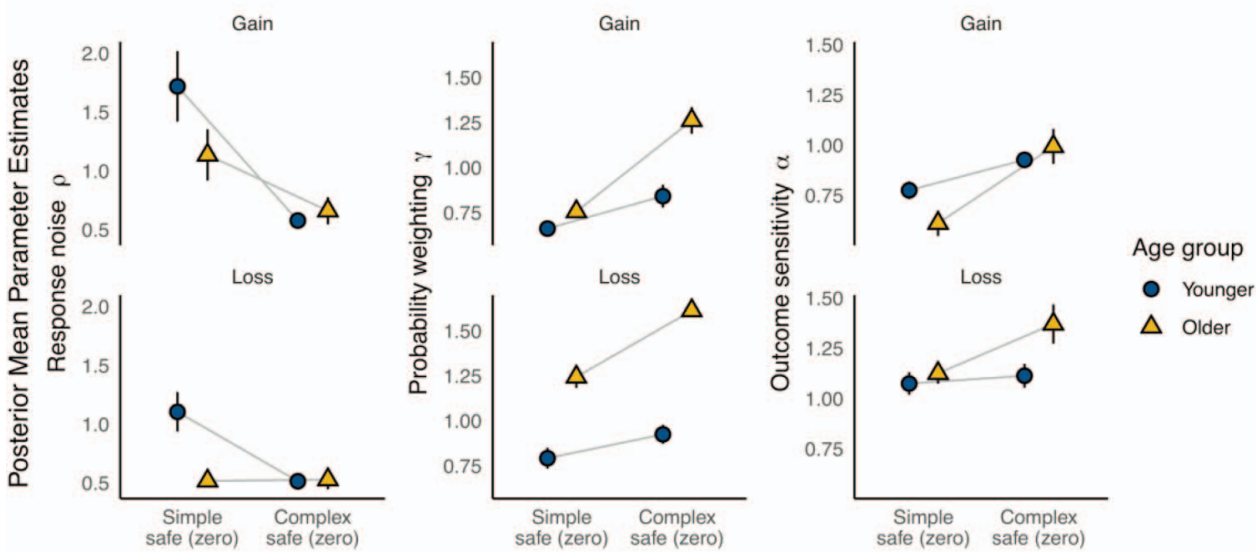

Figure 3. Means and 95\% confidence intervals across the individual-level posterior means for the model parameters $\rho$ (response noise), $\gamma$ (probability weighting), and $\alpha$ (outcome sensitivity), for each condition and age group, separately for the gain and loss domains. See the online article for the color version of this figure.

tion model for both domains. In both domains, the interaction between problem type (complex safe) and age group (older) was credible, indicating that younger adults showed a stronger increase in response noise when the complexity of the safe option was increased. Arguably, this is because older adults already displayed relatively high response noise in the simple safe condition. Taken together, these results support the general notion that choices become less systematic when the complexity of the safe option increases. This effect was more pronounced in younger than older adults. Response noise alone thus cannot explain the directed effect of complexity on the age differences in risky choice behavior: Increasing the complexity of safe options shifts the proportion 
of older adults' safe option choices closer to $50 \%$, but in order for the response noise parameter to explain this pattern, the increase in response noise under higher complexity would have to be more pronounced in older adults, in both domains.

Probability-weighting hypothesis. According to this hypothesis, differences in option complexity distort the shape of the probability-weighting function, and this effect is more pronounced in older than in younger adults. We therefore expected a positive effect of problem type (complex safe) on the probability-weighting parameter $\gamma$ in both domains, and a positive interaction of age group and problem type (complex safe). Figure 3 displays the means and $95 \%$ CIs of the individual-level posterior means for the $\gamma$ parameter and Figure 4 displays the resulting weighting functions for both gains and losses and both age groups. GLM results are displayed in Table 5.

In both domains, there was a credible positive main effect of problem type (complex safe) on $\gamma$, such that the weighting function was less distorted when the second option was a complex safe option than when it was a simple safe option-that is, when the options were more similar in complexity. The interaction between age group and problem type (complex safe) was credible and negative in the domain of gains and credible and positive in the domain of losses. This indicates that with higher option complexity, younger adults showed a stronger increase in the probabilityweighting parameter than older adults in the domain of gains, whereas older adults showed a stronger increase in the probabilityweighting parameter than younger adults in the domain of losses.
These results support the general notion that probability weighting is more linear when options are similarly complex than when they differ in complexity. Further, probability weighting can contribute to explaining the (rather small) effects of complexity on the age differences in risky choice in the domain of losses, but not in the domain of gains. For the probability-weighting parameter to fully explain the choice patterns, older adults would have to show a stronger increase in the probability weighting parameter in both domains.

Outcome-sensitivity hypothesis. According to the outcomesensitivity hypothesis, increasing the complexity of a safe option increases outcome sensitivity, and this effect is more pronounced in older than in younger adults. Figure 3 displays the means of the individual-level posterior distributions of the outcome-sensitivity parameter $\alpha$ and Figure 5 shows the resulting value functions for both domains and age groups. GLM results are displayed in Table 5. There was a positive main effect of problem type (complex safe) on outcome sensitivity in the domain of gains, indicating that outcome sensitivity was higher when the safe and risky option were similarly complex than when the options differed in complexity. The negative main effect of age group in the domain of gains indicates that older adults were generally less sensitive to outcome information than were younger adults. This main effect was not credible in the domain of losses. Importantly, in line with the outcome-sensitivity hypothesis, there was a positive interaction effect of problem type (complex safe) and age group on $\alpha$ in the gain domain, indicating that outcome sensitivity increased more
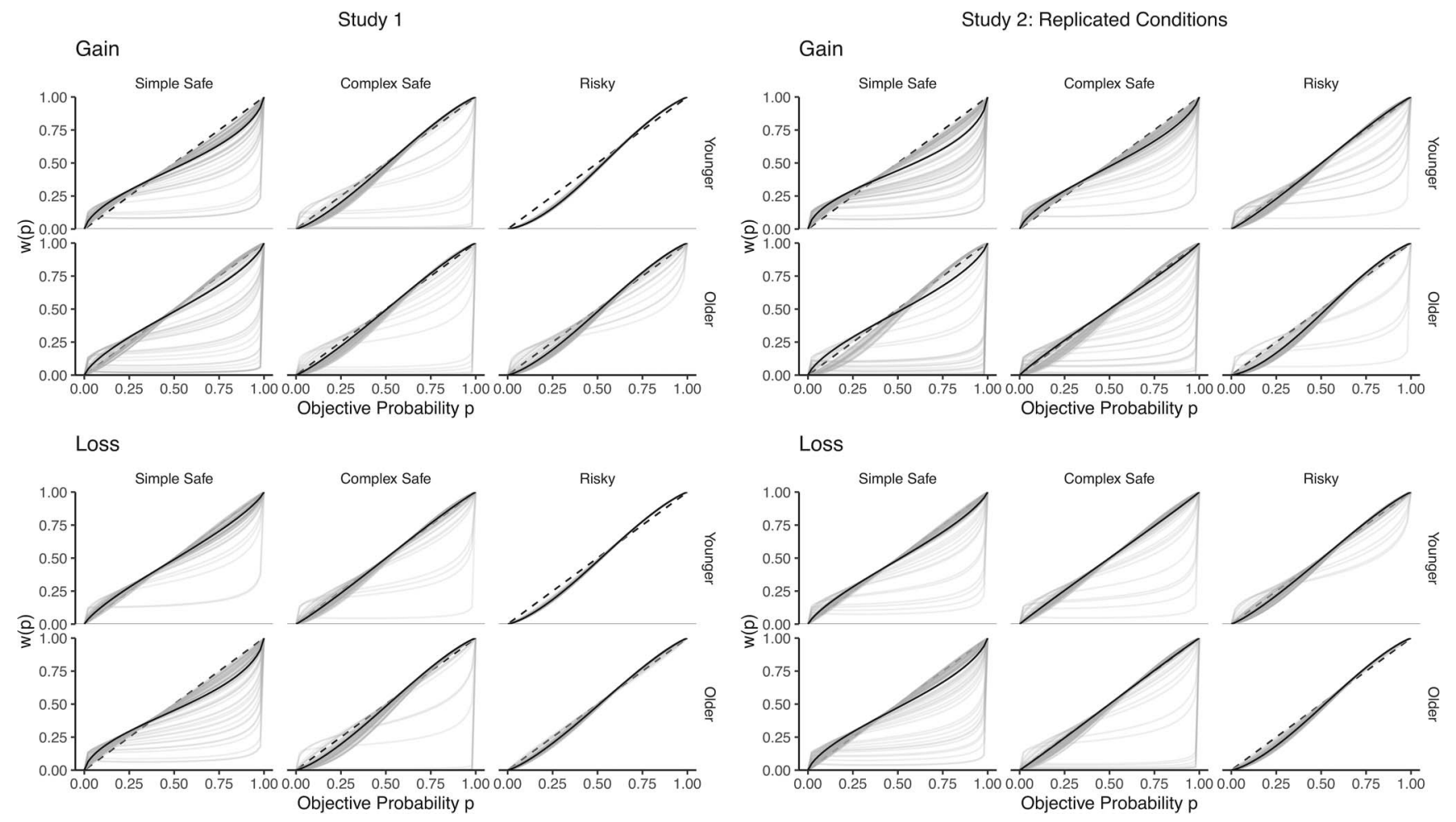

Figure 4. Individual-level probability-weighting functions (based on cumulative prospect theory probabilityweighting parameter estimates for gains and losses) for Study 1 and the corresponding conditions that were replicated in Study 2. 

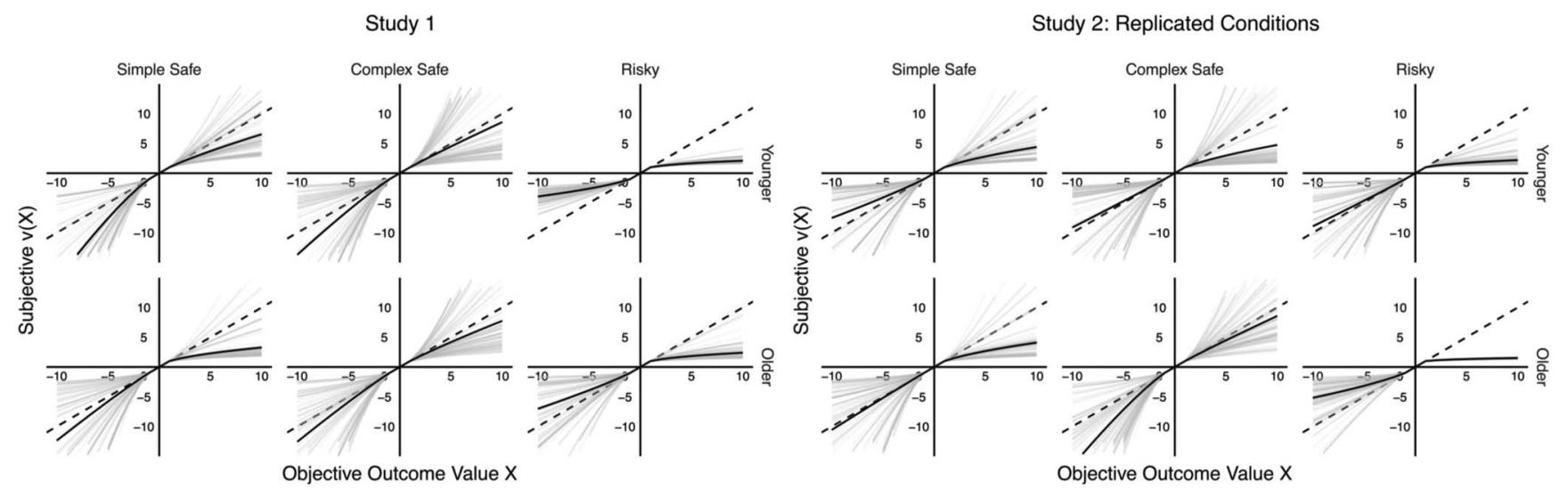

Figure 5. Individual-level value functions (based on cumulative prospect theory outcome-sensitivity parameter estimates for gains and losses) for Study 1 and the corresponding conditions that were replicated in Study 2.

strongly for older adults than for younger adults when both options were similarly complex. This interaction was not credible in the domain of losses.

Like probability weighting, outcome sensitivity seems to contribute to the effects of complexity on age differences in risky choice. However, while outcome sensitivity captured the observed pattern in choice in the domain of gains, it did not do so in losses. In order for the outcome-sensitivity parameter to explain the overall choice patterns, older adults would have to show a stronger increase in the outcome sensitivity parameter in both domains.

In sum, while none of the tested mechanisms could account for the full set of patterns produced by complexity in isolation, the effects on probability weighting and the effects on outcome sensitivity complement each other: When the complexity of safe options was increased, older participants showed a stronger increase in outcome sensitivity in the domain of gains, and a stronger increase in the probability-weighting parameter in the domain of losses relative to younger adults. We thus conclude that complexity seems to affect age differences in risky choice through a combination of mechanisms.

Effect of certainty on CPT parameters beyond an effect of complexity. In order to address predictions from Mather et al.'s (2012) certainty account we also tested whether the availability of a safe option affected the parameters of the CPT analysis after controlling for complexity. To this end, we reran the GLM analyses, this time using the condition with complex safe options as the reference condition. The effect of problem type (risky) in these analyses allowed us to evaluate the isolated effect of certainty. The results are reported in detail in Appendix E. To summarize the key results, there were credible main effects of the availability of a safe option on all parameters of the CPT analysis (except for the response noise parameter in the domain of losses), and, in some cases, of interactions between certainty and age group. That is, even when differences in complexity between safe and risky options were attenuated, the availability of a safe option affected participants' preferences as reflected in CPT.

How do responses in the risky choice task relate to selfreported risk preference? Finally, we explored the relationship between participants' risky choices and their self-reported risk preferences (as measured using the one-item general risk ques- tion). As it turned out, the self-report measure was not related to participants' risky choices in any condition or age group (cf. Table 4 and Tables B1 and B3 in Appendix B). Hence, as in several previous studies (Frey, Pedroni, Mata, Rieskamp, \& Hertwig, 2017; Pedroni et al., 2017), there was a disconnect between behavioral and self-reported measures of risk preference. A Bayesian GLM with self-reported risk preference as the dependent variable and age group and gender as predictors (see Table 6) showed that despite a slight trend toward a decrease in self-reported risk preference in older adults (consistent with findings in large-scale panel data by Dohmen, Falk, Golsteyn, Huffman, \& Sunde, 2017; Josef et al., 2016), this effect was not credible.

\section{Summary of Study 1}

Study 1 provided evidence for a crucial role of differences in option complexity between safe and risky options for the emergence of age differences in risk attitude. As hypothesized, there were age differences in risky choice in problems with simple safe and more complex risky options, but these differences disappeared when safe options were presented in a more complex format. The complexity-aversion hypothesis can be rejected as a potential underlying mechanism, because increasing the complexity of safe losses did not make them less attractive. In addition, modeling the choices with CPT revealed that while higher option complexity increased response noise, this could not explain the differences between younger and older adults in risky choice. Instead, older

Table 6

Regression Coefficients and 95\% Posterior Intervals From the Generalized Linear Model of Self-Reported Risk Preference in Studies 1 and 2

\begin{tabular}{lcc}
\hline \multicolumn{1}{c}{ Predictor } & \multicolumn{1}{c}{ Study 1} & \multicolumn{1}{c}{ Study 2 } \\
\hline \multicolumn{1}{c}{ Outcome variable: Self-reported risk } & preference \\
(Intercept) & $\mathbf{5 . 6 4}[\mathbf{5 . 0 5 , 6 . 2 1}]$ & $\mathbf{4 . 5 4}[\mathbf{3 . 9 7 , 5 . 1 3}]$ \\
Age group (older) & $-0.4[-1.07,0.29]$ & $-0.04[-0.75,0.65]$ \\
Gender (male) & $0.45[-0.28,1.15]$ & $\mathbf{0 . 7}[\mathbf{0 . 0 1}, \mathbf{1 . 4}]$ \\
\hline
\end{tabular}

Note. Boldface indicates credible effects. 
adults' stronger behavioral response to option complexity in the domain of gains was paralleled by a stronger increase in outcome sensitivity compared to younger adults. In the domain of losses, increasing option complexity was associated with a stronger increase in the probability-weighting parameter in older than in younger adults. These findings suggest that the effect of complexity on age differences in risky choice can be captured by a combination of outcome sensitivity and probability weighting, both of which became more linear when complexity differences between options were attenuated.

\section{Study 2}

Whereas Study 1 was conducted online, the goal of Study 2 was to replicate the results in a laboratory experiment. In addition, we extended the investigation of the impact of differences in option complexity on age difference in risky choice to choice problems with a safe option and a risky option featuring a zero outcome-a type of choice problem frequently used in research on age differences in risky choice (e.g., Bruine de Bruin et al., 2007; M. Y. Kim \& Kanfer, 2009; Mamerow et al., 2016; Mather et al., 2012; Mikels \& Reed, 2009; Rönnlund et al., 2005; Rutledge et al., 2016; Thomas \& Millar, 2012; Watanabe \& Shibutani, 2010; Weller et al., 2011). In choice problems in which one risky outcome is zero, complexity differences are arguably smaller than in problems in which the risky option has no zero outcomes. This is because the zero outcome and its probability can be ignored-for instance, a risky option offering a $70 \%$ chance to win $\$ 50$ and a $30 \%$ chance to win $\$ 0$ can be reduced to a $70 \%$ chance to win $\$ 50$, making it similarly complex to a safe option-for instance, offering a $100 \%$ chance to win $\$ 40$. Fuzzy trace theory (Broniatowski \& Reyna, 2018; Reyna \& Brainerd, 1995) also predicts that people are more likely to rely on simplified representations of risky options with outcomes of zero. ${ }^{8}$ Hence, differences in option complexity may play less of a role for age differences in risky choice when a risky outcome of zero is available and increasing the complexity of safe options may therefore also have a lesser effect on age differences in this case. Figure 6 illustrates the new problem types that we used to address this issue. Details on the construction of these problems are provided below. To characterize the participant sample and further increase comparability with other prior research, Study 2 also included some additional cognitive and affective measures (details below).

\section{Method}

Participants. The experiment was conducted at the Max Planck Institute for Human Development in Berlin. Participants were recruited from the Institute's internal participant database. The sample consisted of 80 younger adults and 80 older adults. We approached participants who were either between 18 and 35 years old or at least 60 years old. Demographic characteristics, selfreported risk preference, and cognitive and affective characteristics can be found in Table 7. Participants received a baseline payment of $€ 20$ as well as a performance-contingent monetary bonus. As in Study 1 , the bonus was determined individually for each participant. To induce a realistic sense of the possibility to win or lose money, the experimenter put $€ 5$ in front of the participant as a baseline bonus before starting the experiment. The experimenter explained that the participant's choices in the experiment would determine if they would get to keep the baseline bonus and possibly increase it up to $€ 10$, or whether they would have to return some or even the entire baseline bonus at the end of the experiment. After the risky choice task had been completed, one trial was randomly selected and the option that the participant had chosen was played out. The resulting outcome was converted into euros (with 100 units in the experimental currency E\$, in which the outcomes of the options were presented, corresponding to €5). The converted amount in euros was added to or subtracted from the baseline bonus, depending on whether the randomly selected choice problem was a gain or a loss trial. Detailed instructions about this reward scheme were also provided in written form.

Materials. All tasks of Study 2 were programmed in PsychoPy (Version 1.85.2).

Risky choice task. In addition to the 108 choice problems from Study 1, we included an additional 80 problems consisting of a safe and a two-outcome risky option in which one outcome was zero. Half of these additional problems offered a simple safe option (condition simple safe zero) and the other half offered a complex safe option (condition complex safe zero). The complexity of safe options was manipulated in the same manner as in the original conditions. However, whereas in Study 1 the terms expressing the complex safe outcome did not include zeros, they did in Study 2. For instance, while a safe outcome of 54 might be expressed as $(0.4 \times 90)+(0.6 \times 30) \mathrm{E} \$$ in Study 1 , it might be expressed as $(0.6 \times 90)+(0.4 \times 0) \mathrm{E} \$$ in Study 2 . This served to render complex safe options and risky options with zero outcomes similarly complex on the structural level. In both new conditions, half of the choice problems involved gain outcomes; the other half, loss outcomes. The numerical structure of the new choice problems was based on the unequal-expected-value problems in Mather et al. (2012), meaning that the choice problems were constructed by fixing both options' expected values. The safe option's outcome equaled its expected value, and one of the risky outcomes was set to zero. The nonzero risky outcome was adjusted to conform to the risky option's expected value, while varying its probability from $0.01,0.05,0.1,0.2,0.4,0.6,0.8,0.9,0.95$, to 0.99 across problems. In half of the problems within each condition and domain, the option with the higher expected value was safe; in the other half it was risky. For each of these choice problems, we constructed a version with a simple safe option and a version with a complex safe option. A full list of all choice problems used is provided in the online supplemental materials, where we also report choice proportions of younger and older adults for each individual problem.

Every participant made choices in all conditions and both domains. The choice problems were presented in a randomized order that was uniquely determined for each participant. We also randomized - uniquely for each participant—-the side of the screen on which the high and low risk options appeared on each choice

\footnotetext{
${ }^{8}$ Fuzzy trace theory posits that outcomes of zero allow people to rely on categorical differences ("no money" or "some money") to guide choices, while more sophisticated quantitative comparisons are necessary when such categorically different outcomes are not available (since, for instance, a simplified representation like "some money" or "some money" is not helpful in discriminating between options).
} 

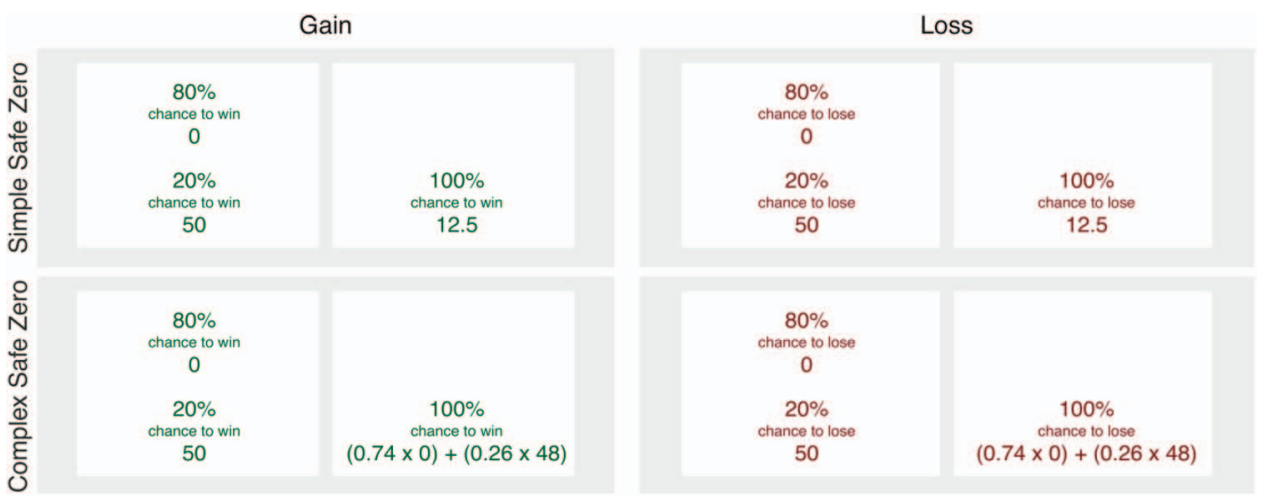

Figure 6. New conditions of the risky choice task in Study 2. See the online article for the color version of this figure.

problem. Response times in the risky choice task were recorded in milliseconds.

Complexity rating, numeracy test, and self-reported risk preference. As in Study 1, participants rated the perceived complexity of a subset of 30 randomly drawn choice problems,

Table 7

Characteristics of the Sample in Study 2 by Age Group

\begin{tabular}{|c|c|c|}
\hline Characteristic & Younger & Older \\
\hline Participants, $N$ & 80 & 80 \\
\hline Gender (female), $n$ & 42 & 41 \\
\hline Age (years) & $26.2(3.9)$ & $70.2(4.8)$ \\
\hline Range (years) & $18-34$ & $61-84$ \\
\hline Self-reported risk preference & $4.9(2.3)$ & $4.8(2.2)$ \\
\hline Numeracy & $2.2(1.2)$ & $1.6(1)$ \\
\hline \multicolumn{3}{|l|}{ Positive affect } \\
\hline Momentary & $3.8(1)$ & $4.8(1.1)$ \\
\hline Habitual & $4.5(1)$ & $5(0.8)$ \\
\hline \multicolumn{3}{|l|}{ Negative affect } \\
\hline Momentary & $1.9(0.8)$ & $1.4(0.6)$ \\
\hline Habitual & $2(0.8)$ & $1.6(0.7)$ \\
\hline \multicolumn{3}{|l|}{ Digit symbol substitution test } \\
\hline$n$ accurate & $56(8.2)$ & $37.3(5.3)$ \\
\hline Proportion accurate & $0.97(0.03)$ & $0.98(0.03)$ \\
\hline \multicolumn{3}{|l|}{ Monthly income, $n$} \\
\hline$\leq € 499$ & 23 & 0 \\
\hline$€ 500-€ 999$ & 26 & 5 \\
\hline$€ 1,000-€ 1,499$ & 16 & 20 \\
\hline$€ 1,500-€ 1,999$ & 6 & 22 \\
\hline$€ 2,000-€ 2,499$ & 3 & 10 \\
\hline$€ 2,500-€ 2,999$ & 0 & 8 \\
\hline$€ 3,000-€ 3,499$ & 0 & 5 \\
\hline$€ 3,500-€ 3,999$ & 0 & 2 \\
\hline$\geq € 4,000$ & 0 & 5 \\
\hline Not disclosed & 6 & 3 \\
\hline \multicolumn{3}{|l|}{ Educational attainment, $n$} \\
\hline Still in school & 1 & 0 \\
\hline \multicolumn{3}{|c|}{ Completion of compulsory basic } \\
\hline secondary school & 0 & 5 \\
\hline Secondary school degree & 3 & 16 \\
\hline High school degree & 25 & 16 \\
\hline Vocational training & 9 & 7 \\
\hline Bachelor degree & 30 & 2 \\
\hline Master degree & 9 & 4 \\
\hline University diploma & 1 & 26 \\
\hline Doctoral degree & 2 & 3 \\
\hline Other & 0 & 1 \\
\hline
\end{tabular}

Note. Data are presented as $M(S D)$ unless otherwise indicated. solved the numeracy test, and indicated their self-reported risk preference.

PANAS. We also included a measure of momentary and habitual affect, a German version of the 10-item Positive and Negative Affect Scale (PANAS; Grühn, Kotter-Grühn, \& Röcke, 2010; Watson, Clark, \& Tellegen, 1988). On each trial of the PANAS, an adjective describing an affective state was presented in the center of the screen and participants were asked to rate how strongly they felt the affect at that moment (for momentary or state affect) or generally (for habitual or trait affect). Participants responded on a 7-point scale (see Grühn et al., 2010). There were two separate blocks for measuring state (momentary) and trait (habitual) affect, both including the same adjectives. The 10 positive and 10 negative adjectives were intermixed and randomized within each block. The order of the two blocks was randomly determined for each participant.

Digit symbol substitution test. We also included a measure of fluid intelligence in terms of speed of processing. Participants completed the digit symbol substitution test (cf. McLeod, Griffiths, Bigelow, \& Yingling, 1982). A table shown on the upper half of the screen defined a (randomly determined, for each participant) mapping between nine symbols and the digits 1-9. On each trial, one of the nine symbols was presented in the center of the screen; participants had to press the associated number key. The next symbol appeared as soon as the participant had responded. The test lasted 90 seconds and participants were instructed to work as quickly and as accurately as possible. We report both the number of correctly matched symbol-number pairs and the percentage of correct responses in Table 7.

Design. The experiment had a mixed design, with age group as between-subjects factor, and type of choice problem (simple safe, complex safe, risky, and the new conditions simple safe zero and complex safe zero) as well as domain (gains vs. losses) as within-subject factors. The experiment was approved by the institutional review board of the Max Planck Institute for Human Development.

Procedure. Upon arriving at the lab, participants were informed about privacy and data-protection guidelines and provided informed consent. Next, participants received instructions regarding the risky choice task and the reward scheme. Participants completed five nonconsequential practice trials before moving on 
to the main part of the task. After the risky choice task, participants completed the complexity rating, the numeracy test, the digit symbol substitution task, and the PANAS. The order of these additional tasks was randomized across participants. After completing all additional tasks, participants indicated their gender, age in years, monthly income, and highest educational attainment, and answered the self-report item on risk preference. They also had the opportunity to comment on the study in an open-answer written format. Each participant's bonus payment amount was automatically determined when they completed the experiment; participants received the baseline plus bonus payment.

\section{Results}

All behavioral analyses were based on the same procedures as in Study 1. As in Study 1, an analysis of participants' choices in problems with a dominated option indicated good data quality: Participants chose the dominating option in $76.88 \%$ of trials in the domain of gains (younger adults: $85.21 \%$; older adults: $68.54 \%$ ) and in $88.07 \%$ of trials in the domain of losses (younger adults: 93.02\%; older adults: $83.12 \%$ ). Further analyses of the choices on the problems with a dominated option are reported in Appendix A.

Was the complexity manipulation successful? As in Study 1 , participants rated the choice problems from the complex safe condition and those from the risky condition as more complex than those from the simple safe condition. Detailed results are reported in Table A1 and illustrated in Figure A2 in Appendix A. Further, problems from the simple safe zero condition were rated as less complex than problems from the simple safe condition. There were no credible differences between complexity ratings for problems from the complex safe zero condition and the simple safe condition. These results show that risky options with a zero outcome are not only structurally less complex than risky options with only nonzero outcomes, but are also perceived as such.

An analysis of the response times showed that, as in Study 1, participants took more time to make choices in the complex safe condition and the risky condition than in the simple safe condition. Moreover, participants made faster choices in the simple safe zero condition than in the simple safe condition, further supporting the notion that the availability of a zero outcome made the choices less difficult. Overall, older adults took more time for their choices than younger adults. An interaction between age group (older) and problem type (complex safe) indicated that older adults' response times increased more than those of younger adults when the complexity of the safe option increased. This held for both gains and losses. Detailed results are reported in the bottom panel of Table A2 and illustrated in Figure A3 in Appendix A.

Did complexity affect age differences in risky choice? Next, we tested whether age differences in the risky choice task were reduced or even eliminated in choices between similarly complex options. The empirical choice proportions of the less risky option in each problem type, domain, and age group are displayed in the top right panel of Figure 2. The patterns in the three replicated conditions closely resemble the findings from Study 1. They replicate, in a different participant sample and experimental setting, the key finding from Study 1 that age differences in risky choice between a safe and a risky option (with two nonzero outcomes) disappear once differences in option complexity are attenuated. These results are reported in detail in Table 4 and Appendix C.

To what extent does this finding extend to the new conditions, in which one of the outcomes of the risky option is zero? In the simple safe zero condition older adults were nominally more likely to choose simple safe gains than were younger adults, but this age difference was smaller than in the simple safe condition without zero outcomes, and not credible. This is consistent with the notion that complexity differences between safe and risky options are reduced when the risky option offers a zero outcome. In the complex safe zero condition, in which the differences in option complexity were further reduced by rendering the safe option more complex, even nominal age differences disappeared in both domains (see Figure 2).

To statistically evaluate these qualitative patterns, we changed the reference level for the factor problem type to the simple safe zero condition, and reran the mixed-effects logistic regressions for risky choice behavior. Coefficients and $95 \%$ posterior intervals are displayed in Table D1 in Appendix D. The interaction between problem type (complex safe zero) and age group (older) was not credible in either domain. Moreover, there was no credible main effect of age group within either condition offering risky outcomes of zero (cf. Table B2 in Appendix B).

To summarize, the results provide further support for our complexity account. In problems in which one of the outcomes of the risky option was zero, and complexity differences were thus comparably low, there were small but not credible age differences in risky choice. These nominal age differences were further reduced by rendering the safe option more complex. Together with the replication of results from Study 1, these findings suggest that age differences observed on the basis of choice problems involving choices between a (simple) safe and a (complex) risky option may not necessarily reflect age differences in risk attitude, but, to some extent, a stronger response to option complexity in older than in younger adults.

Testing the underlying mechanisms. As in Study 1, the complexity aversion hypothesis could be discarded based on the patterns in the risky choice task alone. In the domain of losses, increasing the complexity of safe options made older adults more, rather than less, likely to choose safe options. They found safe options more attractive when their complexity increased-the opposite pattern of that predicted by the complexity aversion hypothesis. Detailed results statistically corroborating this finding are reported in Table B3 in Appendix B.

Next, we again used computational modeling with the same estimation approach and hierarchical Bayesian implementation of CPT as in Study 1 to evaluate the remaining hypotheses, regarding the effects of option complexity on response noise, probability weighting, and outcome sensitivity. The potential scale reduction factor $\hat{R}$ (Gelman \& Rubin, 1992) was smaller than 1.001 for all estimated parameters, indicating very good convergence. The estimated CPT parameters captured the empirical choice patterns well, as indicated by the posterior predictive choice probabilities for each condition, domain, and participant (cf. Figure 2). The means and $95 \%$ CIs of the individual-level posterior means for each parameter of the CPT analysis are shown in Figure 3. The resulting value and weighting functions for the replicated conditions are shown in Figures 4 and 5. The value and weighting functions for the new conditions involving risky outcomes of zero are shown in Figure 7. The CPT-based hypotheses were again 

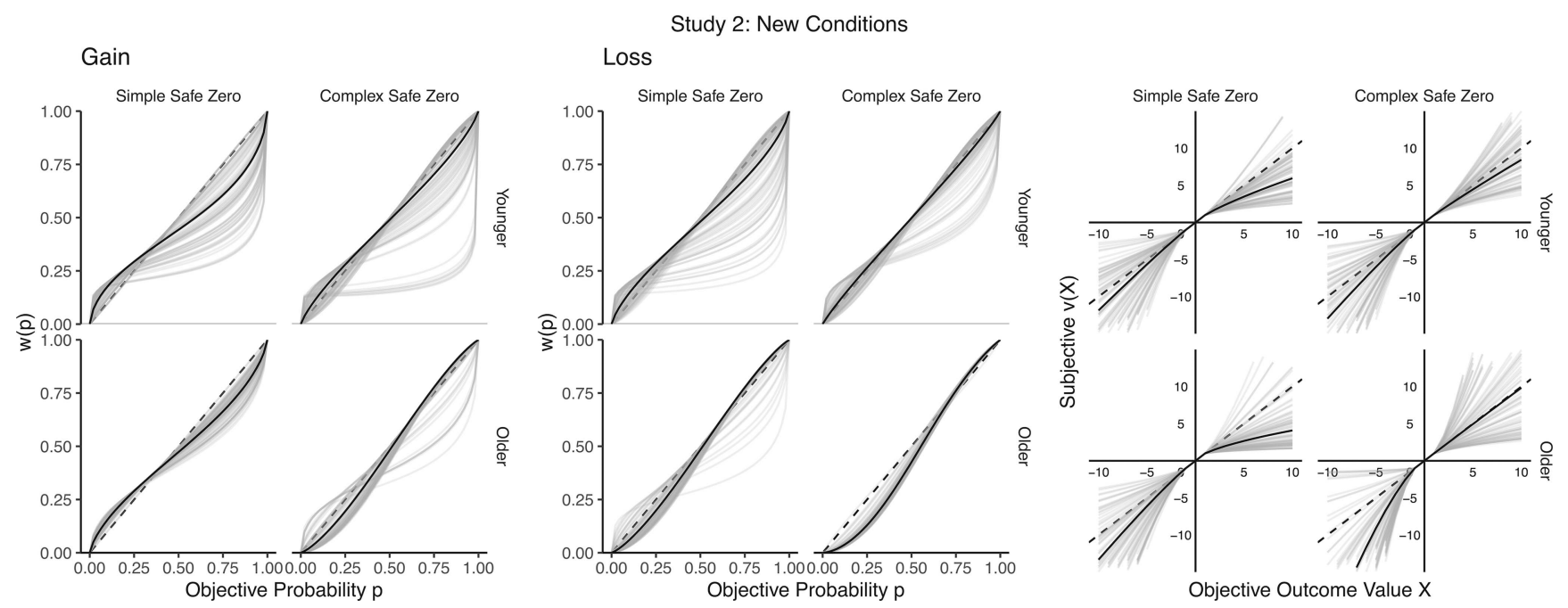

Figure 7. Individual-level probability weighting functions and value functions in the new conditions of Study 2 , involving risky options with zero outcomes. Value and weighting functions are based on cumulative prospect theory outcome-sensitivity parameter estimates and probability-weighting parameter estimates for gains and losses.

evaluated with a series of Bayesian GLM analyses comparing the individual-level parameter estimates in the different conditions and age groups. The coefficients and $95 \%$ highest posterior density intervals for the Bayesian GLMs evaluating these different hypotheses are displayed in Table 8 . We also tested whether the availability of a safe option affected the parameters of the CPT analysis after controlling for complexity. The results are reported in Appendix E.

We first address the results on parameter estimates for the replicated conditions without risky outcomes of zero. Replicating the results from Study 1, response noise was, overall, higher in older than in younger adults in both domains; in the domain of losses, response noise was higher in problems with complex safe options compared to those with simple safe options. In contrast to Study 1, response noise was not credibly higher in problems with complex safe options than in problems with simple safe options in the domain of gains. The interaction between problem type (complex safe) and age group (older) on response noise was not credible, indicating that younger and older adults' response noise was similarly sensitive to an increase in the complexity of safe options.

We now turn to the probability-weighting patterns, which replicated results from Study 1. The positive main effects of problem type (complex safe) in both domains indicate that probabilityweighting functions were less distorted when the problem offered a complex safe option rather than a simple safe option-that is, when the options were more similar in complexity. The interaction between age group and problem type (complex safe) was credible and positive in the domain of losses. This indicates that older adults showed a stronger increase in the probability-weighting parameter than did younger adults in the domain of losses. There was no credible interaction between problem type (complex safe) and age group in the domain of gains.

Finally, and further replicating results from Study 1, outcome sensitivity in the domain of gains was higher when the safe option was complex than when it was simple. In contrast to Study 1, there was a positive main effect of complexity on outcome sensitivity in the domain of losses. We further replicated the result that outcome sensitivity increased more strongly in older adults than in younger adults when safe and risky options were similarly complex in the domain of gains, but not in the domain of losses.

In summary, these modeling results further support the findings in Study 1 that choices are more unsystematic, probability weighting is more linear, and outcome sensitivity is higher when safe and risky options are similarly complex than when they differ in complexity. The results from Study 1 and 2 speak against response noise as an explanation for the effects of option complexity on age differences in risky choice (although option complexity led to more noisy responses overall). Rather, both studies indicate that older participants showed a stronger increase in outcome sensitivity in the domain of gains and a stronger increase in the probability-weighting parameter in the domain of losses relative to younger adults when the complexity of safe options increased. Outcome sensitivity might thus help explain the effects of complexity on age differences in risky choice in the domain of gains, but not losses. The probability-weighting estimates complement this finding, since they help to explain the effects of complexity on age differences in risky choice in the domain of losses (rather than gains).

Impact of complexity on CPT parameters when the risky option has a zero outcome. As described above, in problems in which the risky option had a zero outcome, increasing the complexity of safe options had only a small effect on age differences in risky choice-likely because in such problems the differences in option complexity, and thus age differences in risky choice, were smaller to begin with (relative to problems in which all outcomes of the risky option were nonzero). We nevertheless also examined how the complexity of safe options affected CPT parameters in these problems. To this end, we changed the reference level for the factor condition to the problem type simple safe zero, and reran the Bayesian GLM analyses of CPT parameters. The coefficients are reported in Table 9. The effect of problem type (complex safe 


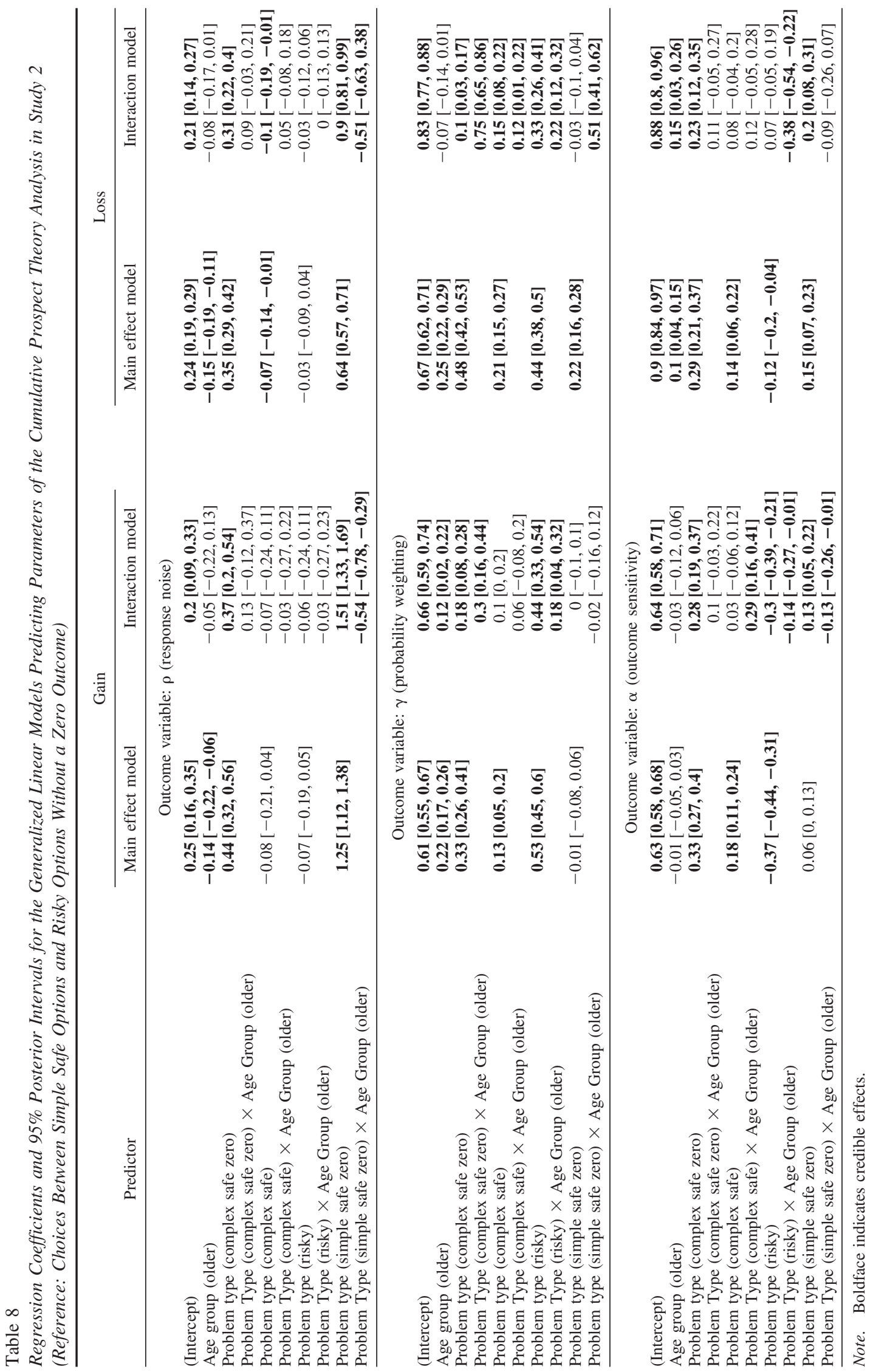




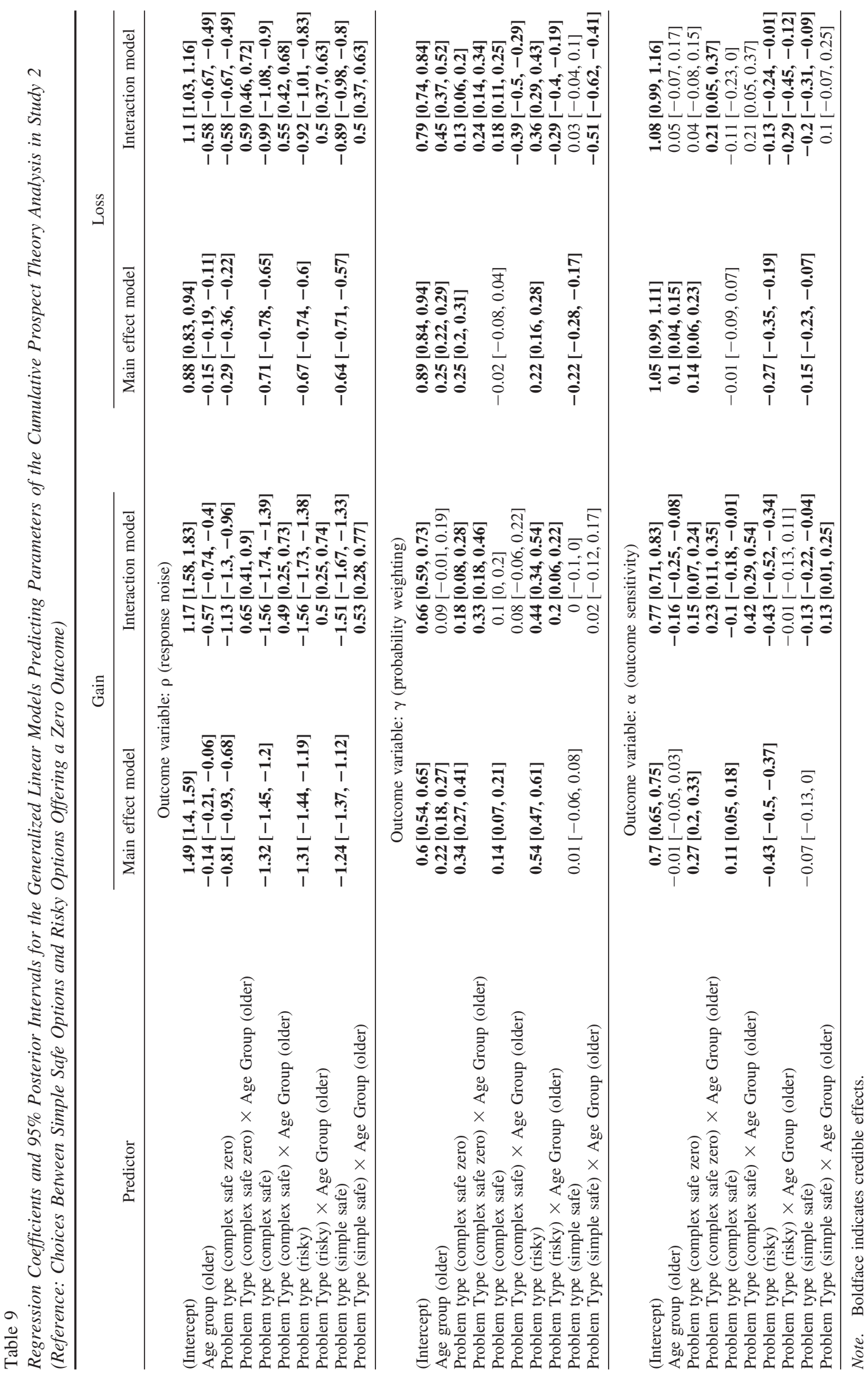


zero) in these analyses allows us to evaluate the effect of safe options' complexity.

There was a credible negative main effect of problem type (complex safe zero) on $\rho$ in both domains, indicating that participants' response noise was higher in choices with a complex safe option than in choices with a simple safe option. This effect was more pronounced in younger than in older adults, indicated by a credible interaction between problem type (complex safe zero) and age group in both domains. There was also a positive main effect of problem type (complex safe zero) on $\gamma$ in both domains indicating that probability weighting was once again more linear when the safe option was complex compared to when it was simple. Moreover, this effect was more pronounced in older than in younger adults, as indicated by the credible positive interactions between problem type (complex safe zero) and age group in both domains. Finally, there was a positive main effect of problem type (complex safe zero) on $\alpha$ in both domains, indicating that outcome sensitivity once more increased when the safe option was complex than when it was simple. There was a credible positive interaction between problem type (complex safe zero) and age group in both domains, suggesting that this effect was more pronounced in older than in younger adults.

Taken together, these results extend the findings from the CPT analyses on the effects of safe options' complexity to choice problems with risky outcomes of zero. Paralleling the results from choice problems without risky outcomes of zero, an increase in the complexity of safe options was associated with a decrease in the response-noise parameter and an increase in both the outcomesensitivity and probability-weighting parameters. The effect of complexity on response noise was less pronounced in older adults, and the effects on probability weighting and on outcome sensitivity were more pronounced in older than in younger adults. That is, in choice problems in which a risky outcome of zero was present, age group and complexity interacted credibly on the level of model parameters-even though the respective patterns in choice behavior were nominally present but fairly small, and not credible.

How do responses in the risky choice task relate to selfreported risk preference? We again explored the relationship between participants' risky choices and their self-reported risk preferences (cf. Table 5 and Tables B2 and B3 in Appendix B). Higher self-reported risk preference was associated with a higher tendency to choose the risky option in the conditions involving safe options in the domain of gains, but not in the conditions involving two risky options, and not in any condition in the domain of losses. As in Study 1, there was no credible main effect of age group on self-reported risk preference (see Table 6).

\section{General Discussion}

Research in psychology and economics on differences in risk attitude between younger and older adults has frequently yielded conflicting results. Many studies have concluded that older adults are more risk averse than younger adults in the gain domain. ${ }^{9}$ This pattern, however, has mainly been obtained in tasks involving a choice between a risky and a safe option (Best \& Charness, 2015; Mather et al., 2012; Rutledge et al., 2016; Tymula et al., 2013). In contrast, when choosing between two risky options, older adults often appear equally or less risk averse than younger adults (Kellen et al., 2017; Pachur et al., 2017). We highlighted that risky and safe options not only differ in risk but also in complexity and proposed that age differences in risky choice might depend on the availability of a safe option, because younger and older adults respond differently to differences in option complexity.

In two studies we varied the complexity of the safe option, thereby rendering the complexity of risky and safe options more similar. The results demonstrated that age differences in risky choice indeed depend strongly on whether options differ in complexity. In the gain domain, older adults were more likely than younger adults to choose a safe option over a risky one when the two options differed in complexity. This age difference disappeared, however, when the differences in complexity were reduced. In the loss domain, older adults were either equally (Study 2) or more (Study 1) likely to choose a risky over a simple safe loss than younger adults; reducing complexity differences eliminated these age differences. The effect of option complexity on age differences in risky choice might help to explain the striking inconsistencies in the literature on age differences in risk attitude.

Examining the underlying mechanism, we showed that these age-dependent effects of complexity were not driven by complexity aversion. Using computational modeling with CPT, we found that increasing the complexity of the safe option has several effects: It introduces more error into the choice process, it increases the sensitivity to differences in outcomes, and it makes probability weighting more linear. Finally, we dissociated the effect of option complexity from an effect of certainty and showed that certainty influences CPT parameters beyond the effect of complexity (see Appendix E). We obtained these findings consistently in an online (Study 1) and a laboratory experiment (Study 2). We found the effects in particular in problems in which people chose between a safe option and a risky option in which all outcomes were nonzero-a situation in which differences in option complexity were rather large. When differences in option complexity were smaller-because the risky option had an outcome of zero- the same qualitative phenomenon emerged, but the effects were weaker and not credible. We now turn to the implications of these findings.

\section{Implications for Age Differences in Decision Making Under Risk}

In this article, we focused on age differences in risk attitude as revealed in a behavioral task, a commonly used approach to investigating decision making under risk (Hertwig, Wulff, \& Mata, 2019; Mata, Frey, Richter, Schupp, \& Hertwig, 2018). Results using this measure have often been interpreted to suggest greater risk aversion in older adults. In contrast, our results suggest that these results may primarily reflect a response to a property of the stimulus-option complexity. Once differences in option complexity are reduced, the age differences in risky choice behavior are reduced or even eliminated.

Let us emphasize, however, that this does not mean that younger and older adults are alike in their risky choices outside the laboratory. First, the level of risk in real-world options may be confounded with complexity. For instance, in many situations a safe and easy-to-evaluate default option may be available (e.g., simply

\footnotetext{
${ }^{9}$ In the domain of losses previous findings are more mixed.
} 
maintaining the status quo). In light of the observed effects of option complexity on choice behavior, age differences are likely to emerge in some natural environments but not in others. As a consequence, it may be difficult (or even impossible) to predict age differences in behavior in risky situations in general. A more modest, and possibly more promising, approach to predicting age differences in risky choice based on behavioral tasks could be to tailor the measurement task to a clearly defined reference class of situations and its contextual features. To this end, it is important to conduct studies like ours, which identify and isolate contextual variables that shape risky choice behavior. The suggested approach also highlights an advantage of behavioral approaches over selfreport measures in studying risk preferences: Contextual features of choice tasks can be explicitly varied to match particular target ecologies and to gauge their impact on behavior.

Second, age differences in risk attitude have also been found beyond behavioral tasks. One major tradition in measuring risk attitude relies on self-reports. For instance, respondents are asked to indicate on a scale from 0 to 10 how prepared they are to take risks in general (Dohmen et al., 2011). A robust finding in studies using this approach is that older adults indicate a lower willingness to take risks than younger adults (Dohmen et al., 2017; Josef et al., 2016; Mata, Josef, \& Hertwig, 2016). It is currently unclear, however, what situations people use to inform their response. Although some variants of commonly used self-report items refer to particular aspects of life-for instance, financial, career, or health risks (Dohmen et al., 2011; Weber, Blais, \& Betz, 2002)the self-report approach to measuring risk preferences affords less control over specific contextual features, rendering it difficult to determine their impact.

In sum, although our understanding of the factors influencing decisions under risk is growing, it may not be possible to derive general conclusions regarding age differences in risky choice behavior, because decisions under risk are sensitive to the structural characteristics of the choice ecology. As a consequence, the predictive power of tasks with specific characteristics (e.g., options differing in complexity) may be limited to only those situations that match them. Acknowledging the characteristics' impact may not only enhance predictive power, but also help explain the modest convergent validity among diverse behavioral measures (Frey et al., 2017; Pedroni et al., 2017).

\section{Can CPT Parameters Be Interpreted Psychologically?}

We used the computational modeling framework of CPT to examine potential mechanisms underlying the effects of complexity on people's choices. In implementations with a probabilistic choice rule, CPT separates random error from systematic transformations of the options' attributes. Moreover, CPT distinguishes between a representation of outcome information (value function) and probability information (weighting function), which together are assumed to shape preferences. Our analyses show that participants displayed more linear probability weighting and higher outcome sensitivity in choice problems involving complex safe rather than simple safe options. What can be inferred from these results about the impact of option complexity on the underlying cognitive processing?

CPT stands in the tradition of "as-if" models of choice, which do not strive to describe the cognitive processes underlying a choice (cf. Berg \& Gigerenzer, 2010). At the same time, key constructs in CPT, such as "loss aversion," "probability sensitivity," and "outcome sensitivity," have been interpreted psychologically (see Pachur, Suter, \& Hertwig, 2017). Several recent analyses have found evidence that CPT - though not modeling cognitive processes themselves - may be systematically linked to how information is processed. For instance, Pachur, Schulte-Mecklenbeck, Murphy, and Hertwig (2018) showed that CPT parameters can reflect the amount of attention allocated to probability and outcome information in a construct-coherent manner (e.g., a more linear probability-weighting function is associated with more time spent attending to probability information). Moreover, Pachur et al. (2017) demonstrated that choices produced by strategies that ignore probability information are reflected in strongly curved probability-weighting functions when modeled with CPT. Finally, probability-weighting patterns may reflect asymmetries in the allocation of attention toward individual options in the choice set during preference formation (Zilker \& Pachur, 2019).

In light of these results, the observed differences in our CPT analyses between the conditions and age groups might point to specific differences in attention allocation. For instance, the more linear probability weighting and higher outcome sensitivity for choices involving complex safe rather than simple safe options may reflect more attention paid to probability and outcome information, or a more symmetric allocation of attention between safe and risky options. Furthermore, patterns in the allocation and impact of attention on preferences may differ between younger and older adults. Addressing these possibilities directly using process tracing (e.g., eye tracking) is an interesting avenue for future research.

\section{Does Higher Option Complexity Increase or Decrease Errors?}

To some extent, the observed effects of option complexity on CPT parameters might seem paradoxical. On the one hand, increasing the complexity of safe options led to more error, measured in terms of response noise, while at the same time it also increased the sensitivity to differences in outcomes and made probability weighting more linear (indicated by less distorted value and weighting functions) - both hallmarks of "normative" preferences.

To resolve this apparent contradiction, it should be noted that the response noise parameter represents nonsystematic errorsthat is, random lapses that cannot be predicted by the model. The probability-weighting function and the value function capture preferences that deviate from an objective treatment of outcomes and probabilities in a systematic and predictable (under CPT) fashion. Crucially, these two types of "error" can occur independent of each other. For instance, even if preferences are perfectly aligned with a linear treatment of outcomes and probabilities, the translation of these preferences into choice behavior can be prone to nonsystematic errors. Likewise, preferences can deviate from a linear treatment of outcomes and probabilities, but be expressed in behavior systematically.

Hence, while the presence of simple safe options makes choices less normative relative to the benchmark of objective attribute processing, it also makes them more predictable under CPT. Conversely, increasing the complexity of safe options increases non- 
systematic errors, but reduces systematic distortions in people's preferences. In this sense, complexity is a double-edged sword: It can either foster or impair adherence to normative standards, depending on which definition of normative standards one subscribes to.

\section{Differential Effects of Complexity in the Gain and Loss Domains}

In Studies 1 and 2, age differences in response to option complexity primarily emerged in the gain domain but were much smaller in the loss domain. In Study 1, the interaction between complexity and age group on risky choice was credible for gains, but not for losses. In Study 2, this interaction was credible in both domains, but there were no credible age differences in the domain of losses when the safe options were simple.

What might explain the differences between domains? Losses have been shown to trigger an increased investment of cognitive resources and attention. For instance, people maximize more, show longer response times, and search more extensively in tasks involving losses rather than gains (e.g., Lejarraga \& Hertwig, 2017; Lejarraga, Schulte-Mecklenbeck, Pachur, \& Hertwig, 2019; Yechiam \& Hochman, 2013). Importantly, this effect might be stronger in older than in younger adults. There is evidence that due to an increasingly unfavorable ratio of gains to losses in later life, older adults undergo a motivational shift in goal orientation and thus focus more strongly on preventing losses rather than on achieving gains (Depping \& Freund, 2011). It has also been demonstrated that such an age-specific motivational shift affects risky choice. Best and Freund (2018) found that older adults were more willing to choose risky options when those options increased the chance of avoiding a larger loss, whereas younger adults were more likely to choose risky options when the chance of obtaining a larger gain increased. An increased focus on loss prevention could motivate older adults to invest more effort and cognitive resources in choices about losses - and thus reduce the impact of option complexity. As a consequence, older and younger adults may behave more similarly in choices about losses than in choices about gains.

\section{Effects of Complexity on Age Differences in Other Risky Choice Paradigms}

We are not the first to demonstrate that differences in cognitive requirements of a task-for instance, due to complexity-affect age differences in risky choice. In their meta-analysis on behavioral risky choice tasks, Mata et al. (2011) concluded that age differences emerged primarily in paradigms with high learning requirements (e.g., because the characteristics of the options have to be learned from experience). Older adults also rely more than their younger counterparts on simpler strategies, which discard certain aspects of information (Mata et al., 2007), especially in choice problems with a high number of options (Besedeš, Deck, Sarangi, \& Shor, 2012a, 2012b). Moreover, a meta-analysis on predecisional information search concluded that older adults search for less information before choosing, especially when options are characterized by a greater number of attributes (Mata \& Nunes, 2010). Similarly, Frey et al. (2015) investigated the effect of choice set size (two, four, or eight options) on age differences in behavior in decisions from experience, where partici- pants learn about options by sampling their payoff distributions. The authors found age differences in the effect of a higher set size on search effort (older adults sampled less per option than younger adults under high set size) but not in choice behavior. This may be due to a subtle but important difference to our study: In contrast to our experiment, where the options within a choice problem differed in complexity, Frey et al. (2015) manipulated the complexity of choice problems as a whole. Taken together, different facets of complexity in risky choice tasks may impact behavior-and age differences therein-in different ways. Consequently, age differences may emerge in response to some, but not necessarily all manifestations of complexity.

\section{Effects of Complexity on Other Decision-Making Phenomena}

Our finding that discrepancies in option complexity seem to crucially shape age differences in decision making may have implications for other prominent decision-making phenomena that are typically demonstrated in tasks with options differing in complexity. One such example are framing effects, and, specifically, preference reversals as a result of different descriptions of otherwise numerically equivalent options (Tversky \& Kahneman, 1981). For instance, people who appear risk averse in choices about positively framed options often appear risk seeking in choices about equivalent, negatively framed options. Studies on framing effects often use tasks (e.g., the Asian disease problem; Tversky \& Kahneman, 1981) that involve a choice between a safe and a risky option, thus giving ample room to the impact of differences in complexity. A second example is loss aversion, the notion that people assign subjectively greater weight to losses than to gains of the same size (Kahneman, Knetsch, \& Thaler, 1991; Tversky \& Kahneman, 1992). Loss aversion has been invoked to explain the observation that most people reject the chance to play a mixed lottery offering equal chances to lose an amount of money and to win an equivalent or even larger amount (Gächter, Johnson, \& Herrmann, 2007; Tom, Fox, Trepel, \& Poldrack, 2007, but see Erev, Ert, \& Yechiam, 2008). Importantly, this task also involves choices between a safe option (i.e., rejecting the risky lottery) and a risky option (i.e., playing the mixed lottery). Finally, option complexity might also affect choices beyond decisions under risk. In intertemporal choice, in which people are asked to choose between a smaller reward sooner or a larger reward later, the immediacy effect describes people's tendency to choose the smaller immediate reward (Keren \& Roelofsma, 1995; Prelec \& Loewenstein, 1991). Immediate rewards, like safe options, tend to be less complex to evaluate. If so, responses to option complexity — rather than immediacy_might play a role in intertemporal choice as well.

Admittedly, this is speculative. But it seems pertinent to systematically examine the extent to which responses to option complexity contribute to classical choice phenomena such as framing effects, loss aversion, and immediacy effects. There is already evidence showing that the presence of safe options increases the magnitude of framing effects (Kühberger, 1998) and contributes to the emergence of loss aversion: Many people show no or little loss aversion in choices between two equally complex risky gambles (Pachur et al., 2017, 2018; Rieskamp, 2008). Potentially, evidence interpreted as an increased susceptibility of older adults to framing effects (e.g., S. Kim, Goldstein, Hasher, \& Zacks, 2005) and 
increased loss aversion (Gächter et al., 2007) may, to some extent, reflect their greater sensitivity to complexity.

\section{Conclusion}

Do risk preferences differ between younger and older adults? A considerable amount of work in psychology and economics has revealed the constructed nature of preferences (Lichtenstein \& Slovic, 2006). To the extent that preferences are constructed, they are likely to be sensitive to contextual features. It has rarely been considered, however, how older and younger adults may differ in their responses to such contextual properties in paradigmatic choice tasks designed to measure risk attitude. We argue that it is essential to acknowledge the influence of subtle task properties on risky choice behavior-otherwise it will remain difficult or even impossible to predict risk behaviors in the wild that are likely to be profoundly impacted by properties of the choice ecology.

\section{References}

Alter, A. L., \& Oppenheimer, D. M. (2009). Uniting the tribes of fluency to form a metacognitive nation. Personality and Social Psychology Review, 13, 219-235. http://dx.doi.org/10.1177/1088868309341564

Baltes, P. B. (1987). Theoretical propositions of life-span developmental psychology: On the dynamics between growth and decline. Developmental Psychology, 23, 611-626. http://dx.doi.org/10.1037/0012-1649 .23.5.611

Becker, A., Deckers, T., Dohmen, T. J., Falk, A., \& Kosse, F. (2012). The relationship between economic preferences and psychological personality measures. Annual Review of Economics, 4, 453-478. http://dx.doi .org/10.1146/annurev-economics-080511-110922

Berg, N., \& Gigerenzer, G. (2010). As-if behavioral economics: Neoclassical economics in disguise? History of Economic Ideas, 18, 133-166. http://dx.doi.org/10.2139/ssrn.1677168

Bernheim, B. D., \& Sprenger, C. (2019). Direct tests of cumulative prospect theory. SSRN. http://dx.doi.org/10.2139/ssrn.3350196

Besedeš, T., Deck, C., Sarangi, S., \& Shor, M. (2012a). Age effects and heuristics in decision making. The Review of Economics and Statistics, 94, 580-595. http://dx.doi.org/10.1162/REST_a_00174

Besedeš, T., Deck, C., Sarangi, S., \& Shor, M. (2012b). Decision-making strategies and performance among seniors. Journal of Economic Behavior \& Organization, 81, 524-533. http://dx.doi.org/10.1016/j.jebo.2011 .07 .016

Best, R., \& Charness, N. (2015). Age differences in the effect of framing on risky choice: A meta-analysis. Psychology and Aging, 30, 688-698. http://dx.doi.org/10.1037/a0039447

Best, R., \& Freund, A. M. (2018). Age, loss minimization, and the role of probability for decision-making. Gerontology, 64, 475-484. http://dx .doi.org/10.1159/000487636

Broniatowski, D. A., \& Reyna, V. F. (2018). A formal model of fuzzy-trace theory: Variations on framing effects and the Allais paradox. Decision, 5, 205-252. http://dx.doi.org/10.1037/dec0000083

Broomell, S. B., \& Bhatia, S. (2014). Parameter recovery for decision modeling using choice data. Decision, 1, 252-274. http://dx.doi.org/10 $.1037 / \mathrm{dec} 0000020$

Bruine de Bruin, W., Parker, A. M., \& Fischhoff, B. (2007). Individual differences in adult decision-making competence. Journal of Personality and Social Psychology, 92, 938-956. http://dx.doi.org/10.1037/00223514.92.5.938

Charness, G., \& Gneezy, U. (2012). Strong evidence for gender differences in risk taking. Journal of Economic Behavior \& Organization, 83, 50-58. http://dx.doi.org/10.1016/j.jebo.2011.06.007
Cokely, E. T., Galesic, M., Schulz, E., Ghazal, S., \& Garcia-Retamero, R. (2012). Measuring risk literacy: The Berlin Numeracy Test. Judgment and Decision Making, 7, 25-47.

Craik, F. I. M., \& Bialystok, E. (2006). Cognition through the lifespan: Mechanisms of change. Trends in Cognitive Sciences, 10, 131-138. http://dx.doi.org/10.1016/j.tics.2006.01.007

Curtis, C. E., \& D'Esposito, M. (2003). Persistent activity in the prefrontal cortex during working memory. Trends in Cognitive Sciences, 7, 415423. http://dx.doi.org/10.1016/S1364-6613(03)00197-9

Deakin, J., Aitken, M., Robbins, T., \& Sahakian, B. J. (2004). Risk taking during decision-making in normal volunteers changes with age. Journal of the International Neuropsychological Society, 10, 590-598. http://dx .doi.org/10.1017/S1355617704104104

Depping, M. K., \& Freund, A. M. (2011). Normal aging and decision making: The role of motivation. Human Development, 54, 349-367. http://dx.doi.org/10.1159/000334396

D’Esposito, M., Detre, J. A., Alsop, D. C., Shin, R. K., Atlas, S., \& Grossman, M. (1995). The neural basis of the central executive system of working memory. Nature, 378, 279-281. http://dx.doi.org/10.1038/ $378279 \mathrm{a} 0$

Dohmen, T., Falk, A., Golsteyn, B. H. H., Huffman, D., \& Sunde, U. (2017). Risk attitudes across the life course. Economic Journal, 127, F95-F116. http://dx.doi.org/10.1111/ecoj.12322

Dohmen, T., Falk, A., Huffman, D., \& Sunde, U. (2018). On the relationship between cognitive ability and risk preference. The Journal of Economic Perspectives, 32, 115-134. http://dx.doi.org/10.1257/jep.32.2 .115

Dohmen, T., Falk, A., Huffman, D., Sunde, U., Schupp, J., \& Wagner, G. G. (2011). Individual risk attitudes: Measurement, determinants, and behavioral consequences. Journal of the European Economic Association, 9, 522-550. http://dx.doi.org/10.1111/j.1542-4774.2011.01015.x

Dror, I. E., Katona, M., \& Mungur, K. (1998). Age differences in decision making: To take a risk or not? Gerontology, 44, 67-71. http://dx.doi .org/10.1159/000021986

Erev, I., Ert, E., \& Yechiam, E. (2008). Loss aversion, diminishing sensitivity, and the effect of experience on repeated decisions. Journal of Behavioral Decision Making, 21, 575-597. http://dx.doi.org/10.1002/ bdm.602

Frey, R., Mata, R., \& Hertwig, R. (2015). The role of cognitive abilities in decisions from experience: Age differences emerge as a function of choice set size. Cognition, 142, 60-80. http://dx.doi.org/10.1016/j .cognition.2015.05.004

Frey, R., Pedroni, A., Mata, R., Rieskamp, J., \& Hertwig, R. (2017). Risk preference shares the psychometric structure of major psychological traits. Science Advances, 3, e1701381. http://dx.doi.org/10.1126/sciadv .1701381

Gabry, J., Simpson, D., Vehtari, A., Betancourt, M., \& Gelman, A. (2019). Visualization in Bayesian workflow. Journal of the Royal Statistical Society Series A, Statistics in Society, 182, 389-402. http://dx.doi.org/ 10.1111/rssa. 12378

Gächter, S., Johnson, E. J., \& Herrmann, A. (2007). Individual-level loss aversion in riskless and risky choices (IZA Discussion Paper Number 2961). Retrieved from http://ftp.iza.org/dp2961.pdf

Gelman, A., \& Rubin, D. B. (1992). Inference from iterative simulation using multiple sequences. Statistical Science, 7, 457-472. http://dx.doi .org/10.1214/ss/1177011136

Glöckner, A., Hilbig, B. E., Henninger, F., \& Fiedler, S. (2016). The reversed description-experience gap: Disentangling sources of presentation format effects in risky choice. Journal of Experimental Psychology: General, 145, 486-508. http://dx.doi.org/10.1037/a0040103

Goodrich, B., Gabry, J., Ali, I., \& Brilleman, S. (2018). rstanarm: Bayesian applied regression modeling via Stan (R package Version 2.18.2) [Computer software]. Retrieved from https://cran.r-project.org/ package $=$ rstanarm 
Grühn, D., Kotter-Grühn, D., \& Röcke, C. (2010). Discrete affects across the adult lifespan: Evidence for multidimensionality and multidirectionality of affective experiences in young, middle-aged and older adults. Journal of Research in Personality, 44, 492-500. http://dx.doi.org/10 .1016/j.jp. 2010.06.003

Henninger, D. E., Madden, D. J., \& Huettel, S. A. (2010). Processing speed and memory mediate age-related differences in decision making. Psychology and Aging, 25, 262-270. http://dx.doi.org/10.1037/a0019096

Hertwig, R., Wulff, D. U., \& Mata, R. (2019). Three gaps and what they may mean for risk preference. Philosophical Transactions of the Royal Society B: Biological Sciences, 374, 1766. http://dx.doi.org/10.1098/rstb .2018 .0140

Holt, C. A., \& Laury, S. K. (2002). Risk aversion and incentive effects. The American Economic Review, 92, 1644-1655. http://dx.doi.org/10.1257/ 000282802762024700

Horn, J. L., \& Cattell, R. B. (1967). Age differences in fluid and crystallized intelligence. Acta Psychologica, 26, 107-129. http://dx.doi.org/10 .1016/0001-6918(67)90011-X

Huck, S., \& Weizsäcker, G. (1999). Risk, complexity, and deviations from expected-value maximization: Results of a lottery choice experiment. Journal of Economic Psychology, 20, 699-715. http://dx.doi.org/10 .1016/S0167-4870(99)00031-8

Josef, A. K., Richter, D., Samanez-Larkin, G. R., Wagner, G. G., Hertwig, R., \& Mata, R. (2016). Stability and change in risk-taking propensity across the adult life span. Journal of Personality and Social Psychology, 111, 430-450. http://dx.doi.org/10.1037/pspp0000090

Kahneman, D., Knetsch, J. L., \& Thaler, R. H. (1991). Anomalies: The endowment effect, loss aversion, and status quo bias. The Journal of Economic Perspectives, 5, 193-206. http://dx.doi.org/10.1257/jep.5.1 .193

Kahneman, D., \& Tversky, A. (1979). Prospect theory: An analysis of decision under risk. Econometrica, 47, 263-292. http://dx.doi.org/10 .2307/1914185

Kellen, D., Mata, R., \& Davis-Stober, C. P. (2017). Individual classification of strong risk attitudes: An application across lottery types and age groups. Psychonomic Bulletin \& Review, 24, 1341-1349. http://dx.doi .org/10.3758/s13423-016-1212-5

Kellen, D., Pachur, T., \& Hertwig, R. (2016). How (in)variant are subjective representations of described and experienced risk and rewards? Cognition, 157, 126-138. http://dx.doi.org/10.1016/j.cognition.2016.08 .020

Keren, G., \& Roelofsma, P. (1995). Immediacy and certainty in intertemporal choice. Organizational Behavior and Human Decision Processes, 63, 287-297. http://dx.doi.org/10.1006/obhd.1995.1080

Kim, M. Y., \& Kanfer, R. (2009). The joint influence of mood and a cognitively demanding task on risk-taking. Motivation and Emotion, 33, 362-372. http://dx.doi.org/10.1007/s11031-009-9147-z

Kim, S., Goldstein, D., Hasher, L., \& Zacks, R. T. (2005). Framing effects in younger and older adults. The Journals of Gerontology: Series B, 60, 215-218. http://dx.doi.org/10.1093/geronb/60.4.P215

Knight, F. H. (1921). Risk, uncertainty and profit. Boston, MA: Houghton Mifflin Company.

Kovář́k, J., Levin, D., \& Wang, T. (2016). Ellsberg paradox: Ambiguity and complexity aversions compared. Journal of Risk and Uncertainty, 52, 47-64. http://dx.doi.org/10.1007/s11166-016-9232-0

Krawczyk, D. C. (2002). Contributions of the prefrontal cortex to the neural basis of human decision making. Neuroscience and Biobehavioral Reviews, 26, 631-664. http://dx.doi.org/10.1016/S01497634(02)00021-0

Kruschke, J. (2014). Doing Bayesian data analysis: A tutorial with $R$, $J A G S$, and Stan. London, United Kingdom: Academic Press.

Kühberger, A. (1998). The influence of framing on risky decisions: A meta-analysis. Organizational Behavior and Human Decision Processes, 75, 23-55. http://dx.doi.org/10.1006/obhd.1998.2781
Lauriola, M., \& Levin, I. P. (2001). Personality traits and risky decisionmaking in a controlled experimental task: An exploratory study. Personality and Individual Differences, 31, 215-226. http://dx.doi.org/10 .1016/S0191-8869(00)00130-6

Lee, M. D. (2011). How cognitive modeling can benefit from hierarchical Bayesian models. Journal of Mathematical Psychology, 55, 1-7. http:// dx.doi.org/10.1016/j.jmp.2010.08.013

Lee, T. M. C., Leung, A. W. S., Fox, P. T., Gao, J.-H., \& Chan, C. C. H. (2008). Age-related differences in neural activities during risk taking as revealed by functional MRI. Social Cognitive and Affective Neuroscience, 3, 7-15. http://dx.doi.org/10.1093/scan/nsm033

Lejarraga, T., \& Hertwig, R. (2017). How the threat of losses makes people explore more than the promise of gains. Psychonomic Bulletin \& Review, 24, 708-720. http://dx.doi.org/10.3758/s13423-016-1158-7

Lejarraga, T., Schulte-Mecklenbeck, M., Pachur, T., \& Hertwig, R. (2019). The attention-aversion gap: How allocation of attention relates to loss aversion. Evolution and Human Behavior, 40, 457-469. http://dx.doi .org/10.1016/j.evolhumbehav.2019.05.008

Li, S.-C., Lindenberger, U., \& Sikström, S. (2001). Aging cognition: From neuromodulation to representation. Trends in Cognitive Sciences, 5, 479-486. http://dx.doi.org/10.1016/S1364-6613(00)01769-1

Lichtenstein, S., \& Slovic, P. (Eds.). (2006). The construction of preference. Cambridge, United Kingdom: Cambridge University Press. http:// dx.doi.org/10.1017/CBO9780511618031

Lopes, L. L. (1987). Between hope and fear: The psychology of risk. In L. Berkowitz (Ed.), Advances in experimental social psychology (Vol. 20, pp. 255-295). San Diego, CA: Elsevier. http://dx.doi.org/10.1016/ S0065-2601(08)60416-5

Mador, G., Sonsino, D., \& Benzion, U. (2000). On complexity and lotteries' evaluation-Three experimental observations. Journal of Economic Psychology, 21, 625-637. http://dx.doi.org/10.1016/S0167-4870(00) 00023-4

Mamerow, L., Frey, R., \& Mata, R. (2016). Risk taking across the life span: A comparison of self-report and behavioral measures of risk taking. Psychology and Aging, 31, 711-723. http://dx.doi.org/10.1037/ pag0000124

Markowitz, H. (1952). Portfolio selection. The Journal of Finance, 7 , 77-91.

Mata, R., Frey, R., Richter, D., Schupp, J., \& Hertwig, R. (2018). Risk preference: A view from psychology. The Journal of Economic Perspectives, 32, 155-172. http://dx.doi.org/10.1257/jep.32.2.155

Mata, R., Josef, A. K., \& Hertwig, R. (2016). Propensity for risk taking across the life span and around the globe. Psychological Science, 27, 231-243. http://dx.doi.org/10.1177/0956797615617811

Mata, R., Josef, A. K., Samanez-Larkin, G. R., \& Hertwig, R. (2011). Age differences in risky choice: A meta-analysis. Annals of the New York Academy of Sciences, 1235, 18-29. http://dx.doi.org/10.1111/j.17496632.2011.06200.x

Mata, R., \& Nunes, L. (2010). When less is enough: Cognitive aging, information search, and decision quality in consumer choice. Psychology and Aging, 25, 289-298. http://dx.doi.org/10.1037/a0017927

Mata, R., Schooler, L. J., \& Rieskamp, J. (2007). The aging decision maker: Cognitive aging and the adaptive selection of decision strategies Psychology and Aging, 22, 796-810. http://dx.doi.org/10.1037/08827974.22.4.796

Mather, M., Mazar, N., Gorlick, M. A., Lighthall, N. R., Burgeno, J., Schoeke, A., \& Ariely, D. (2012). Risk preferences and aging: The "certainty effect" in older adults' decision making. Psychology and Aging, 27, 801-816. http://dx.doi.org/10.1037/a0030174

Mayhorn, C. B., Fisk, A. D., \& Whittle, J. D. (2002). Decisions, decisions: Analysis of age, cohort, and time of testing on framing of risky decision options. Human Factors, 44, 515-521. http://dx.doi.org/10.1518/ 0018720024496935 
McLeod, D. R., Griffiths, R. R., Bigelow, G. E., \& Yingling, J. (1982). An automated version of the digit symbol substitution test (DSST). Behavior Research Methods and Instrumentation, 14, 463-466. http://dx.doi.org/ 10.3758/BF03203313

Mikels, J. A., \& Reed, A. E. (2009). Monetary losses do not loom large in later life: Age differences in the framing effect. The Journals of Gerontology: Series B, 64, 457-460. http://dx.doi.org/10.1093/geronb/ gbp043

Morey, R. D., Hoekstra, R., Rouder, J. N., Lee, M. D., \& Wagenmakers, E.-J. (2016). The fallacy of placing confidence in confidence intervals. Psychonomic Bulletin \& Review, 23, 103-123. http://dx.doi.org/10 .3758/s13423-015-0947-8

Murphy, R. O., \& ten Brincke, R. H. W. (2018). Hierarchical maximum likelihood parameter estimation for cumulative prospect theory: Improving the reliability of individual risk parameter estimates. Management Science, 64, 308-326. http://dx.doi.org/10.1287/mnsc.2016.2591

Nilsson, H., Rieskamp, J., \& Wagenmakers, E.-J. (2011). Hierarchical Bayesian parameter estimation for cumulative prospect theory. Journal of Mathematical Psychology, 55, 84-93. http://dx.doi.org/10.1016/j.jmp .2010 .08 .006

Olschewski, S., Rieskamp, J., \& Scheibehenne, B. (2018). Taxing cognitive capacities reduces choice consistency rather than preference: A model-based test. Journal of Experimental Psychology: General, 147, 462-484. http://dx.doi.org/10.1037/xge0000403

Pachur, T., Mata, R., \& Hertwig, R. (2017). Who dares, who errs? Disentangling cognitive and motivational roots of age differences in decisions under risk. Psychological Science, 28, 504-518. http://dx.doi.org/10 $.1177 / 0956797616687729$

Pachur, T., Schulte-Mecklenbeck, M., Murphy, R. O., \& Hertwig, R. (2018). Prospect theory reflects selective allocation of attention. Journal of Experimental Psychology: General, 147, 147-169. http://dx.doi.org/ 10.1037/xge0000406

Pachur, T., Suter, R. S., \& Hertwig, R. (2017). How the twain can meet: Prospect theory and models of heuristics in risky choice. Cognitive Psychology, 93, 44-73. http://dx.doi.org/10.1016/j.cogpsych.2017.01.001

Pedroni, A., Frey, R., Bruhin, A., Dutilh, G., Hertwig, R., \& Rieskamp, J. (2017). The risk elicitation puzzle. Nature Human Behaviour, 1, 803809. http://dx.doi.org/10.1038/s41562-017-0219-x

Prelec, D., \& Loewenstein, G. (1991). Decision making over time and under uncertainty: A common approach. Management Science, 37, 770786. http://dx.doi.org/10.1287/mnsc. 37.7 .770

QuestBack GmbH. (2016). Unipark [Computer software]. Retrieved from https://www.unipark.com

Reyna, V. F., \& Brainerd, C. J. (1995). Fuzzy-trace theory: An interim synthesis. Learning and Individual Differences, 7, 1-75. http://dx.doi .org/10.1016/1041-6080(95)90031-4

Rieskamp, J. (2008). The probabilistic nature of preferential choice. Journal of Experimental Psychology: Learning, Memory, and Cognition, 34, 1446-1465. http://dx.doi.org/10.1037/a0013646

Rönnlund, M., Karlsson, E., Laggnäs, E., Larsson, L., \& Lindström, T. (2005). Risky decision making across three arenas of choice: Are younger and older adults differently susceptible to framing effects? The Journal of General Psychology, 132, 81-92. http://dx.doi.org/10.3200/ GENP.132.1.81-93

Rottenstreich, Y., \& Hsee, C. K. (2001). Money, kisses, and electric shocks: On the affective psychology of risk. Psychological Science, 12, 185-190. http://dx.doi.org/10.1111/1467-9280.00334

Rutledge, R. B., Smittenaar, P., Zeidman, P., Brown, H. R., Adams, R. A., Lindenberger, U., . . . Dolan, R. J. (2016). Risk taking for potential reward decreases across the lifespan. Current Biology, 26, 1634-1639. http://dx.doi.org/10.1016/j.cub.2016.05.017

Rypma, B., \& D'Esposito, M. (2000). Isolating the neural mechanisms of age-related changes in human working memory. Nature Neuroscience, 3, 509-515. http://dx.doi.org/10.1038/74889
Rypma, B., Prabhakaran, V., Desmond, J. E., \& Gabrieli, J. D. E. (2001) Age differences in prefrontal cortical activity in working memory. Psychology and Aging, 16, 371-384. http://dx.doi.org/10.1037/08827974.16.3.371

Salat, D. H., Tuch, D. S., Hevelone, N. D., Fischl, B., Corkin, S., Rosas, H. D., \& Dale, A. M. (2005). Age-related changes in prefrontal white matter measured by diffusion tensor imaging. Annals of the New York Academy of Sciences, 1064, 37-49. http://dx.doi.org/10.1196/annals.1340.009

Salthouse, T. A. (2004). What and when of cognitive aging. Current Directions in Psychological Science, 13, 140-144. http://dx.doi.org/10 .1111/j.0963-7214.2004.00293.x

Scheibehenne, B., \& Pachur, T. (2015). Using Bayesian hierarchical parameter estimation to assess the generalizability of cognitive models of choice. Psychonomic Bulletin \& Review, 22, 391-407. http://dx.doi.org/ 10.3758/s13423-014-0684-4

Sonsino, D., Benzion, U., \& Mador, G. (2002). The complexity effects on choice with uncertainty-Experimental evidence. Economic Journal, 112, 936-965. http://dx.doi.org/10.1111/1468-0297.00073

Stott, H. P. (2006). Cumulative prospect theory's functional menagerie. Journal of Risk and Uncertainty, 32, 101-130. http://dx.doi.org/10.1007/ s11166-006-8289-6

Su, Y.-S., \& Yajima, M. (2015). R2jags: Using r to run "JAGS" (R package Version 0.5-7) [Computer software]. Retrieved from http://CRAN.Rproject.org/package $=$ R2jags

Thomas, A. K., \& Millar, P. R. (2012). Reducing the framing effect in older and younger adults by encouraging analytic processing. The Journals of Gerontology: Series B, 67, 139-149. http://dx.doi.org/10.1093/ geronb/gbr076

Tom, S. M., Fox, C. R., Trepel, C., \& Poldrack, R. A. (2007). The neural basis of loss aversion in decision-making under risk. Science, 315, 515-518. http://dx.doi.org/10.1126/science.1134239

Tversky, A., \& Kahneman, D. (1981). The framing of decisions and the psychology of choice. Science, 211, 453-458. http://dx.doi.org/10.1126/ science.7455683

Tversky, A., \& Kahneman, D. (1992). Advances in prospect theory: Cumulative representation of uncertainty. Journal of Risk and Uncertainty, 5, 297-323. http://dx.doi.org/10.1007/BF00122574

Tymula, A., Rosenberg Belmaker, L. A., Ruderman, L., Glimcher, P. W., \& Levy, I. (2013). Like cognitive function, decision making across the life span shows profound age-related changes. Proceedings of the $\mathrm{Na}$ tional Academy of Sciences, 110, 17143-17148. http://dx.doi.org/10 .1073/pnas.1309909110

Watanabe, S., \& Shibutani, H. (2010). Aging and decision making: Differences in susceptibility to the risky-choice framing effect between older and younger adults in Japan. The Japanese Psychological Research, 52, 163 174. http://dx.doi.org/10.1111/j.1468-5884.2010.00432.x

Watson, D., Clark, L. A., \& Tellegen, A. (1988). Development and validation of brief measures of positive and negative affect: The PANAS scales. Journal of Personality and Social Psychology, 54, 1063-1070. http://dx.doi.org/10.1037/0022-3514.54.6.1063

Weber, E. U., Blais, A.-R., \& Betz, N. E. (2002). A domain-specific risk-attitude scale: Measuring risk perceptions and risk behaviors. Journal of Behavioral Decision Making, 15, 263-290. http://dx.doi.org/10 $.1002 / \mathrm{bdm} .414$

Weber, E. U., Shafir, S., \& Blais, A.-R. (2004). Predicting risk sensitivity in humans and lower animals: Risk as variance or coefficient of variation. Psychological Review, 111, 430-445. http://dx.doi.org/10.1037/ 0033-295X.111.2.430

Weller, J. A., Levin, I. P., \& Denburg, N. L. (2011). Trajectory of risky decision making for potential gains and losses from ages 5 to 85. Journal of Behavioral Decision Making, 24, 331-344. http://dx.doi.org/10.1002/ bdm. 690 
West, R. L. (1996). An application of prefrontal cortex function theory to cognitive aging. Psychological Bulletin, 120, 272-292. http://dx.doi.org/ 10.1037/0033-2909.120.2.272

Yechiam, E., \& Hochman, G. (2013). Loss-aversion or loss-attention: The impact of losses on cognitive performance. Cognitive Psychology, 66, 212-231. http://dx.doi.org/10.1016/j.cogpsych.2012.12.001

Zamarian, L., Sinz, H., Bonatti, E., Gamboz, N., \& Delazer, M. (2008). Normal aging affects decisions under ambiguity, but not decisions under risk. Neuropsychology, 22, 645-657. http://dx.doi.org/10.1037/0894-4105.22.5.645
Zaval, L., Li, Y., Johnson, E. J., \& Weber, E. U. (2015). Complementary contributions of fluid and crystallized intelligence to decision making across the life span. In T. M. Hess, J. Strough, \& C. E. Löckenhoff (Eds.), Aging and decision making (pp. 149-168). San Diego, CA: Elsevier. http://dx.doi.org/10.1016/B978-0-12-417148-0 $.00008-\mathrm{X}$

Zilker, V., \& Pachur, T. (2019). Signatures of attention in risky choice: Linking attentional drift diffusion models and cumulative prospect theory. Manuscript in preparation.

\section{Appendix A}

\section{Choices in Problems With a Dominant Option and Manipulation Checks}

Participants' choices of the dominant option in both studies were analyzed with Bayesian GLMERs, including problem type, age group, their interaction, expected-value differences, and numeracy scores as fixed effects, and a random intercept for each participant. The results are displayed in the top panel of Table A1 (Study 1) and Table A2 (Study 2) and illustrated in Figure A1. The negative main effect of problem type (complex safe) indicates that participants were more likely to choose the dominant option in the problems with simple safe options (in both domains in Study 2 and in the loss domain in Study 1). In both studies and across both domains, participants with higher numeracy scores were more likely to choose the dominant option. In Study 1, younger and older adults did not differ in their choice of the dominant option, and in Study 2 older adults were less likely to choose the dominant option, in both domains. There were no credible interactions between problem type and age group in either study or domain.

We also used Bayesian GLMERs to analyze participants' complexity ratings of the different types of choice problems including problem type, age group, their interaction, expected value difference, self-reported risk preferences and numeracy scores as fixed effects, and a random intercept for each participant. Results from the analysis of complexity ratings are displayed in the middle panel of Table A1 (Study 1) and Table A2 (Study 2) and illustrated in Figure A2. In both studies and domains, participants rated problems from the complex safe condition and from the risky condition as more complex than problems from the simple safe condition. In Study 2, participants rated problems with a zero outcome in the domain of gains as less complex compared to the corresponding problem type that did not involve zero outcomes. In addition, problems with higher expected value differences between the options were rated as less complex, and problems in which the higher expected value option was riskier were perceived as more complex. In Study 1, there was no credible main effect of age group on the complexity ratings, indicating that viewed across all conditions, older and younger adults did not differ in their perception of complexity. In Study 2, the credible positive main effect of age group in the domain of gains indicates that overall, older adults rated problems as more complex. There was a credible negative interaction between problem type (complex safe) and age group in the domain of gains (Study 2) and in the domain of losses (Study 1 ), indicating that older adults' complexity ratings increased less than those of younger adults in the condition with complex safe compared to simple safe options.

Finally, we used Bayesian GLMERs to analyze participants' response times on the nondominated choice problems. These models included problem type, age group, their interaction, a binary variable indicating whether the option with the higher expected value was also more risky, expected-value difference, numeracy scores, and self-reported risk preference as fixed effects, and a random intercept for each participant. Results are displayed in the bottom panel of Table A1 (Study 1) and Table A2 (Study 2) and illustrated in Figure A3. Participants took more time to respond in the complex safe condition and in the risky condition, compared to the simple safe condition. Overall, older adults took longer to make choices than did younger adults. In Study 1, participants with higher numeracy scores also generally took more time to make choices in the domain of gains. On trials with larger expected value differences (which are easier) response times were shorter in the domain of gains (both studies) and the domain of losses (Study 2). Finally, an interaction between problem type (complex safe) and age group (older) indicates that older adults' response times increased more substantially when the complexity of safe options increased than did the response times of younger adults (Study 2). In Study 2, participants took less time on choice problems with risky options offering a zero outcome compared to the corresponding problems where no zero outcome was available. 


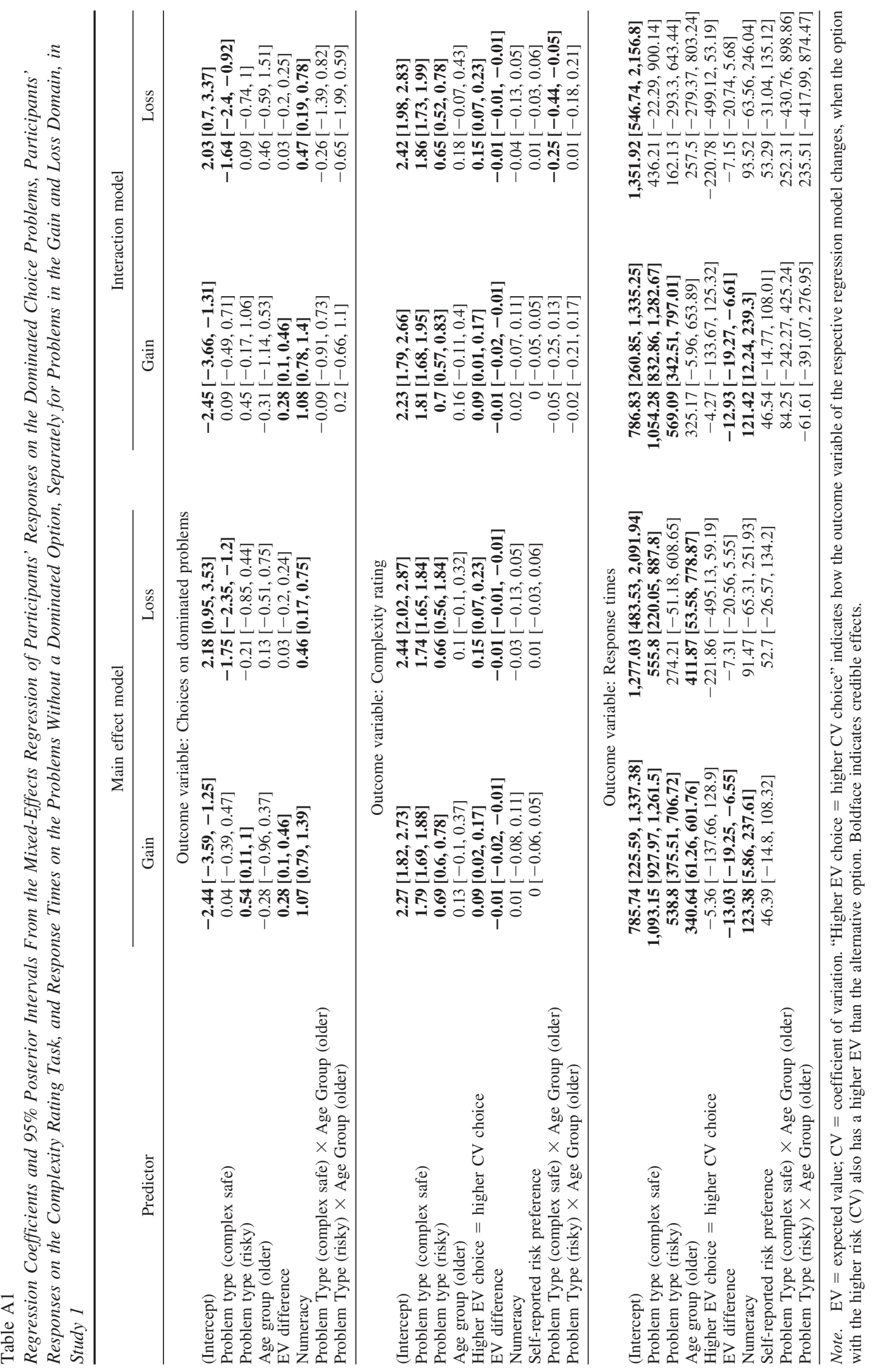



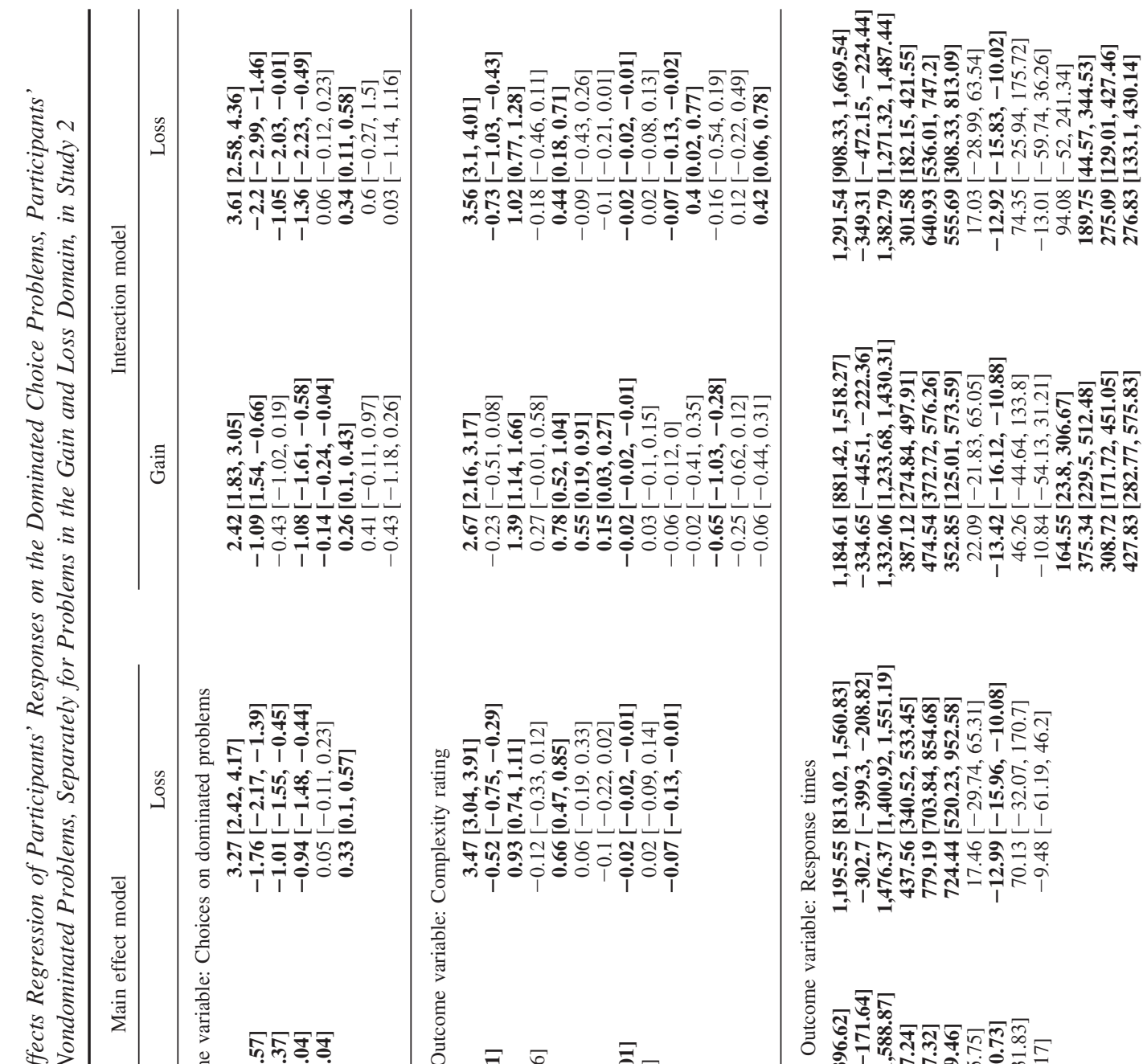

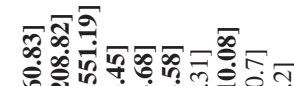

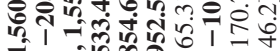

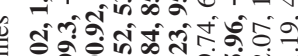

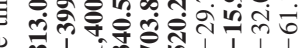

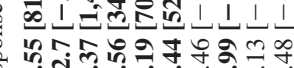

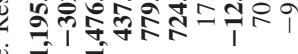

$\frac{0}{\circ}$

氶

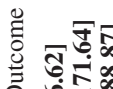

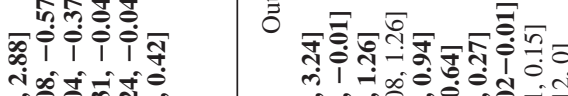

तิ

Ti门

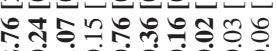

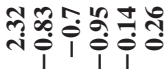

i

悹
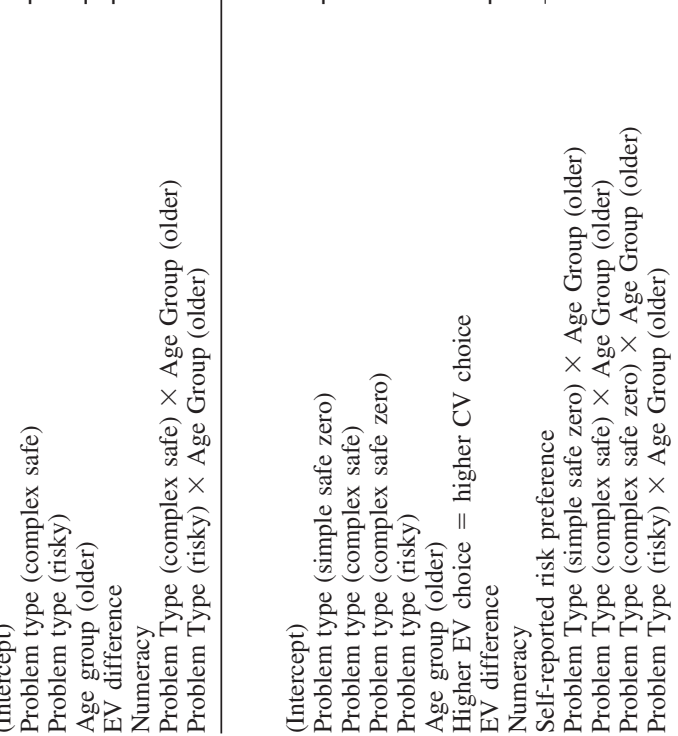

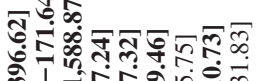

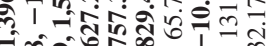

o 01000 -

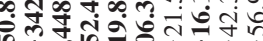

至

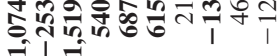


Study 1

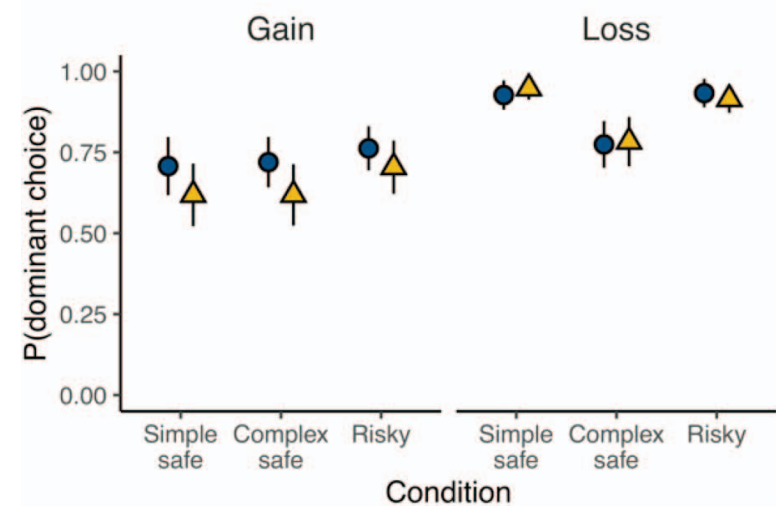

Study 2

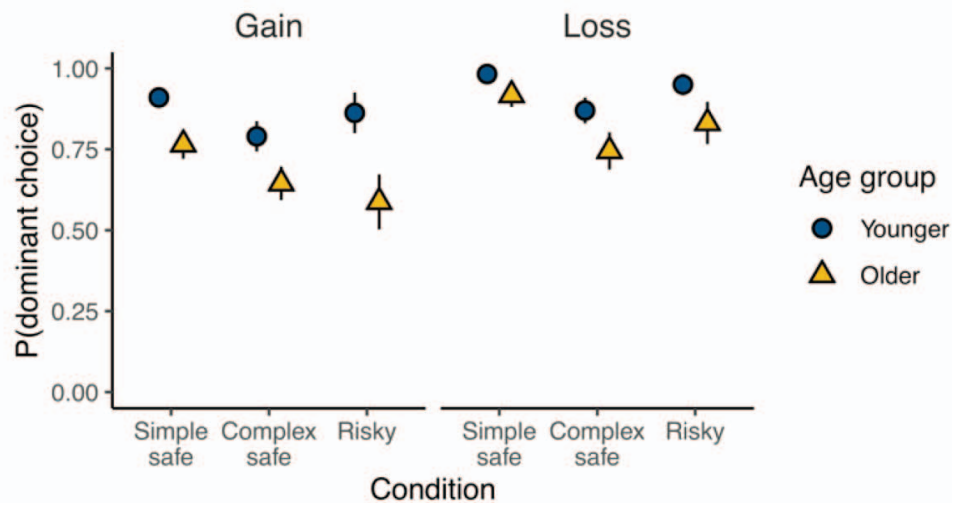

Figure A1. Choice proportions for the dominated problems in all conditions and age groups by domain in Study 1 and Study 2. Error bars indicate 95\% confidence intervals. See the online article for the color version of this figure.

Study 1

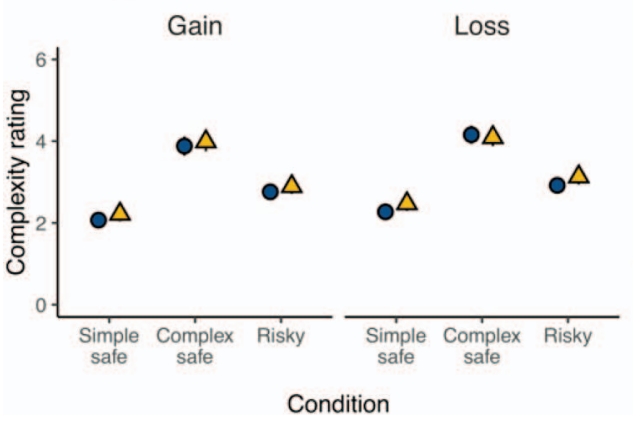

Study 2

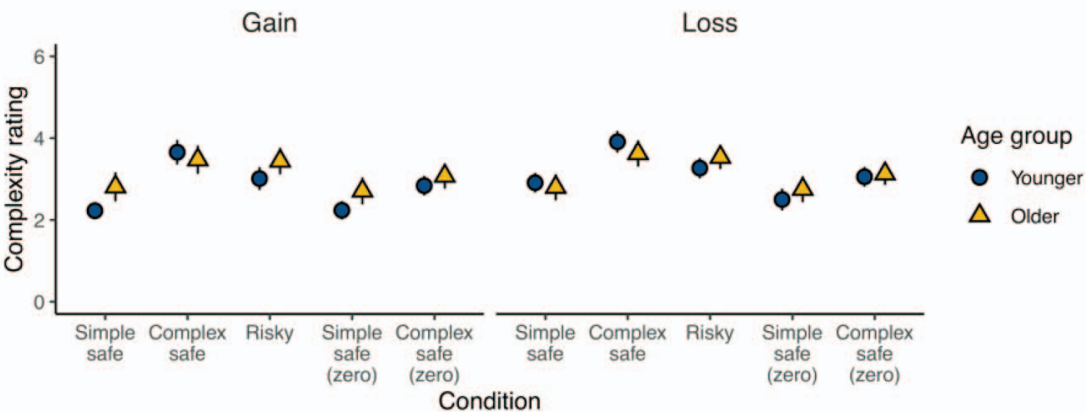

Figure A2. Complexity ratings for nondominated problems in all conditions and age groups by domain in Study 1 and Study 2. Error bars indicate 95\% confidence intervals. See the online article for the color version

Study 1 of this figure.

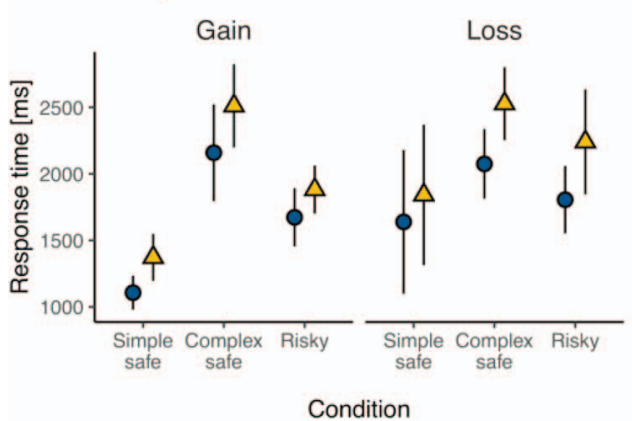

Study 2

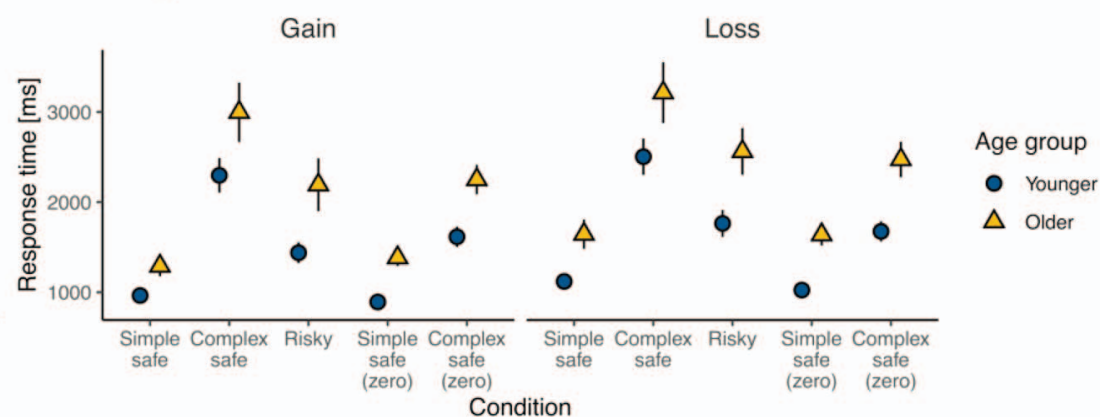

Figure A3. Response times for nondominated problems in all conditions and age groups by domain in Study 1 and Study 2. Error bars indicate 95\% confidence intervals. See the online article for the color version of this figure. 


\section{Appendix B}

\section{Analysis of Risky Choice Patterns Within Conditions and Age Groups}

We conducted Bayesian mixed-effects logistic regressions to predict the choice of the riskier option, within each individual condition, using age group as a fixed effect. The models further included fixed effects for the expected value difference between the options, a dummy variable indicating whether the option with the higher expected value was also riskier, each participant's numeracy score, and their self-reported risk preference and gender. The models included a random intercept for each participant. Results for condition-wise analyses are displayed in Table B1 for Study 1 and Table B2 for Study 2. In choices between simple safe and risky options, older adults were credibly less risk seeking than younger adults in the domain of gains (both studies) and credibly more risk seeking than younger adults in the domain of losses (Study 1). In the condition with complex safe options and the condition with two risky options, there were no credible age differences in either study (except in the risky condition in the gain domain in Study 2). This supports our hypothesis that age differences in risk attitude are reduced or eliminated when options are similarly complex.

We also conducted Bayesian mixed-effects logistic regressions to predict the choice of the riskier option, within each individual age group, using condition as a fixed effect. The models further included fixed effects for the expected value difference between the options, a dummy variable indicating whether the option with the higher expected value was also riskier, each participant's numeracy score, and their self-reported risk preference and gender. The model included a random intercept for each participant. Results for the analyses by age group are displayed in Table B3. In both studies, older adults were more likely to choose the riskier option in choices between complex safe and risky options and in choices between two risky options compared to choices with simple safe options, in the domain of gains. In the domain of losses, older adults were less likely to choose the riskier option in choices between complex safe and risky options (Study 2) and in choices between two risky options (both studies), compared to the condition with simple safe options. Younger adults' behavior tended to change in the same directions, but the effects were weaker or not credible. This further supports the hypothesis that older adults are more sensitive to complexity differences than younger adults. 

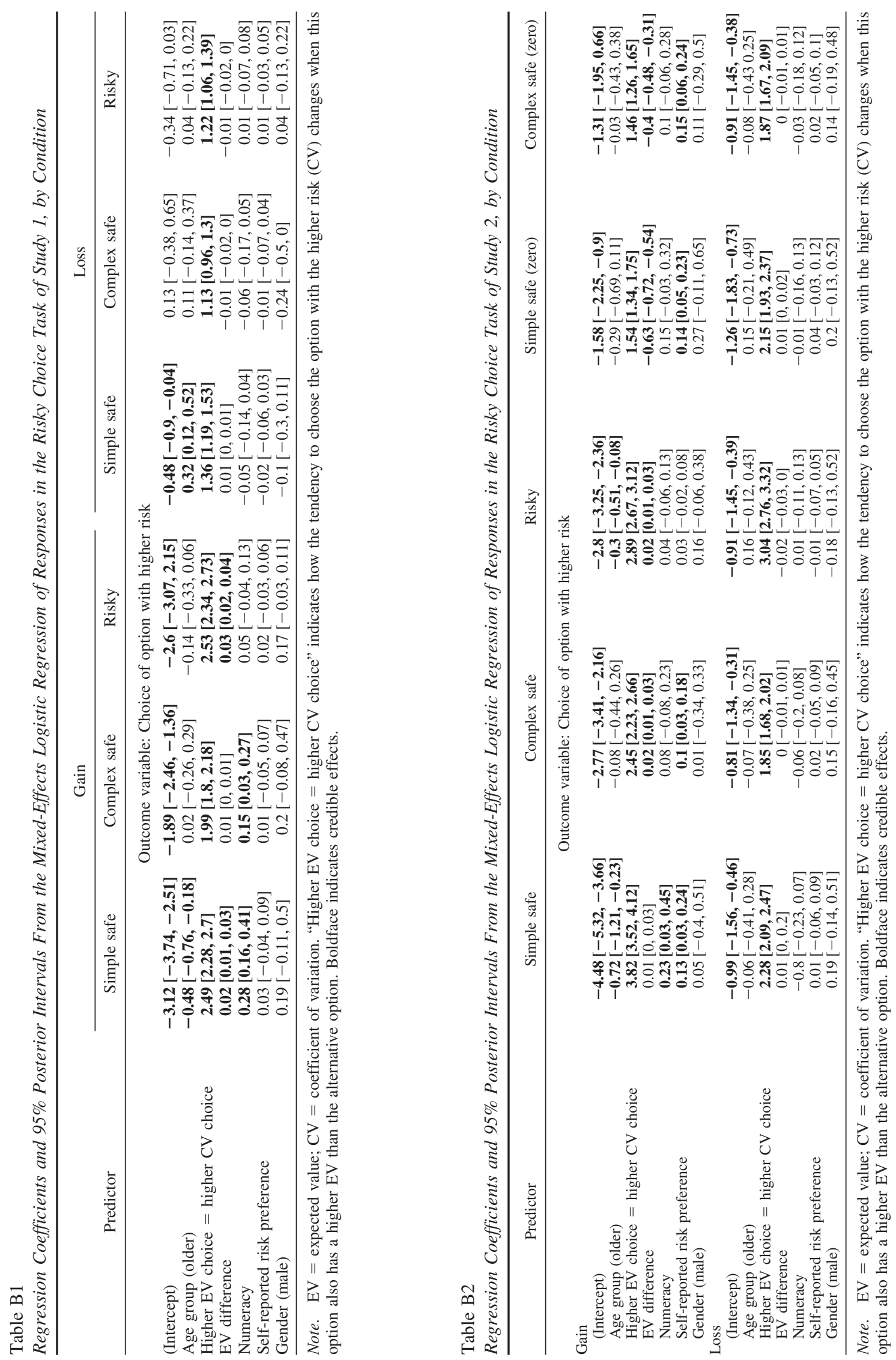
Table B3

Regression Coefficients and 95\% Posterior Intervals From the Mixed-Effects Logistic Regression of Responses in the Risky Choice Task of Studies 1 and 2, by Age Group

\begin{tabular}{|c|c|c|c|c|}
\hline \multirow[b]{2}{*}{ Predictor } & \multicolumn{2}{|c|}{ Gain } & \multicolumn{2}{|c|}{ Loss } \\
\hline & Younger & Older & Younger & Older \\
\hline \multicolumn{5}{|c|}{ Outcome variable: Choice of option with higher risk } \\
\hline \multicolumn{5}{|l|}{ Study 1} \\
\hline (Intercept) & $2.35[-2.85,-1.84]$ & $-3.28[-3.87,-2.7]$ & $-0.46[-0.85,-0.08]$ & $0.3[-0.14,0.73]$ \\
\hline Problem type (complex safe) & $0.19[0.01,0.38]$ & $0.69[0.49,0.88]$ & $0.03[-0.14,0.2]$ & $-0.16[-0.33,0.01]$ \\
\hline Problem type (risky) & $0.06[-0.13,0.23]$ & $0.44[0.24,0.63]$ & $0.06[-0.1,0.23]$ & $-0.24[-0.4,-0.08]$ \\
\hline Higher $\mathrm{EV}$ choice $=$ higher $\mathrm{CV}$ choice & $2.33[2.17,2.48]$ & $2.26[2.09,2.42]$ & $1.33[1.19,1.47]$ & $1.12[0.98,1.26]$ \\
\hline EV difference & $0.01[0.01,0.02]$ & $0.02[0.01,0.03]$ & $0[-0.01,0]$ & $-0.01[-0.01,0]$ \\
\hline Numeracy & $0.16[0.07,0.27]$ & $0.14[0,0.27]$ & $0.04[-0.04,0.12]$ & $-0.1[-0.21,-0.01]$ \\
\hline Self-reported risk preference & $-0.01[-0.07,0.04]$ & $0.06[-0.01,0.13]$ & $-0.01[-0.05,0.03]$ & $-0.01[-0.07,0.05]$ \\
\hline Gender (male) & $0.2[-0.05,0.42]$ & $0.1[-0.21,0.42]$ & $-0.2[-0.38,-0.01]$ & $-0.02[-0.25,0.23]$ \\
\hline \multicolumn{5}{|l|}{ Study 2} \\
\hline (Intercept) & $-3.03[-3.52,-2.52]$ & $-3.05[-3.67,-2.47]$ & $-0.41[-0.93,0.09]$ & $-0.81[-1.32,-0.28]$ \\
\hline Problem type (simple safe zero) & $0.16[-0.08,0.4]$ & $0.42[0.19,0.66]$ & $-0.42[-0.65,-0.18]$ & $-0.44[-0.65,0.23]$ \\
\hline Problem type (complex safe) & $0.31[0.13,0.5]$ & $0.75[0.56,0.94]$ & $-0.07[-0.27,0.13]$ & $-0.38[-0.56,-0.2]$ \\
\hline Problem type (complex safe zero) & $0.65[0.41,0.88]$ & $1.1[0.88,1.34]$ & $-0.42[-0.65,-0.18]$ & $0.38[-0.59,-0.16]$ \\
\hline Problem type (risky) & $0.23[0.05,0.43]$ & $0.53[0.34,0.72]$ & $-0.16[-0.35,0.04]$ & $-0.21[-0.39,-0.03]$ \\
\hline Higher $\mathrm{EV}$ choice $=$ higher $\mathrm{CV}$ choice & $2.4[2.28,2.53]$ & $1.77[1.65,1.89]$ & $2.46[2.33,2.58]$ & $1.97[1.85,2.08]$ \\
\hline EV difference & $0.01[0.01,0.02]$ & $0.01[0,0.02]$ & $-0.01[-0.01,0]$ & $-0.01[-0.01,0]$ \\
\hline Numeracy & $0.04[-0.07,0.17]$ & $0.21[0.02,0.42]$ & $-0.14[-0.27,-0.01]$ & $0.11[-0.07,0.29]$ \\
\hline Self-reported risk preference & $0.11[0.05,0.18]$ & $0.08[-0.02,0.17]$ & $0.01[-0.06,0.08]$ & $0.01[-0.07,0.09]$ \\
\hline Gender (male) & $0.29[0,0.59]$ & $-0.09[-0.48,0.33]$ & $-0.05[-0.38,0.28]$ & $0.27[-0.08,0.61]$ \\
\hline
\end{tabular}

Note. $\quad \mathrm{EV}=$ expected value; $\mathrm{CV}=$ coefficient of variation. "Higher $\mathrm{EV}$ choice $=$ higher $\mathrm{CV}$ choice" indicates how the tendency to choose the option with the higher risk (CV) changes when this option also has a higher EV than the alternative option. Boldface indicates credible effects.

\section{Appendix C}

\section{Analysis of Risky Choice Patterns in the Replicated Conditions of Study 2}

We tested whether the behavioral results from the three conditions of the risky choice task in Study 1 were replicated in Study 2. Based on the corresponding conditions in Study 2, we tested whether age differences in risky choice behavior were reduced or even eliminated in choices between similarly complex options. Empirical choice proportions of the less risky option in each problem type, domain, and age group are displayed in the top panel of Figure 2.

As can be seen, in the condition with simple safe options in the domain of gains older adults appear more risk averse than younger adults. These age differences are attenuated in the other conditions, in which options are more similarly complex. In the domain of losses, younger and older adults are similarly risk seeking in the condition with simple safe options, and both age groups showed reduced risk seeking in the conditions with similarly complex options. This increase in safe choices in the complex safe condition compared to the simple safe condition in the domain of losses is more pronounced in older adults. Bayesian GLMER analyses establish the statistical credibility of these patterns (for coefficients and $95 \%$ posterior intervals, see Table 4 ).
Whereas in Study 1 the interaction between problem type (complex safe) and age group was only credible in the domain of gains, in Study 2 this interaction was credible in both domains. Hence, Study 2 provides further evidence in favor of our basic hypothesis that older adults' choices are more sensitive to differences in option complexity between safe and risky options than younger adults', this time across both domains (though the effect is still stronger for gains than for losses). Further analyses testing for the main effect of age group within each condition further support the notion that age differences in risky choice behavior are reduced or eliminated when both options are similarly complex (see Table B2 in Appendix B).

Further replicating results from Study 1, the behavioral patterns discard the complexity-aversion hypothesis: Increasing the complexity of safe options made older adults more likely to choose safe options in the domain of losses. Older adults found safe options credibly more attractive when their complexity increased - which cannot be explained by complexity aversion. Detailed results statistically corroborating this finding are reported in Table B3 in Appendix B. 


\section{Appendix D}

\section{Analysis of Risky Choice Problems Offering a Risky Outcome of Zero}

In Study 2, we included two new conditions to test for a positive (negative) interaction between age and option complexity on the tendency to choose the option with the higher risk when a risky outcome of zero was available in the domain of gains (losses). To test for these effects, we conducted Bayesian mixed-effects logistic regressions to predict the choice of the riskier option in Study 2, using problem type and age group (main effect model) as well as their interaction (interaction model) as fixed effects. For these models we changed the reference level for the factor problem type to the simple safe zero condition. The models further included fixed effects for the expected-value difference between options, a dummy variable indicating whether the option with the higher ex- pected value was also riskier, and each participant's numeracy score, gender, and self-reported risk preference. Coefficients and 95\% posterior intervals are displayed in Table D1. There was no credible interaction between problem type (complex safe zero) and age group in either domain. Note that in choices between simple safe options and risky options with zero outcomes (which are more similar in their complexity, compared to choices with simple safe options and risky options without zero outcomes) age differences were nominally present but not credible. Hence, rendering the options even more similar in their complexity by increasing the complexity of safe options could at most further reduce these nominal age differences in risky choice behavior.

Table D1

Regression Coefficients and 95\% Posterior Intervals From the Bayesian Mixed-Effects Logistic Regression for Responses in the Risky Choice Task in Study 2 (Reference Condition: Simple Safe Zero)

\begin{tabular}{|c|c|c|c|c|}
\hline \multirow[b]{2}{*}{ Predictor } & \multicolumn{2}{|c|}{ Main effect model } & \multicolumn{2}{|c|}{ Interaction model } \\
\hline & Gain & Loss & Gain & Loss \\
\hline \multicolumn{5}{|c|}{ Outcome variable: Choice of option with higher risk } \\
\hline (Intercept) & $-2.65[-3.07,-2.23]$ & $-1.04[-1.44,-0.64]$ & $-2.64[-3.06,-2.2]$ & $-1.05[-1.47,-0.66]$ \\
\hline Problem type (simple safe) & $-0.29[-0.45,-0.13]$ & $0.43[0.26,0.59]$ & $-0.16[-0.36,0.05]$ & $0.4[0.2,0.6]$ \\
\hline Problem type (complex safe) & $0.25[0.08,0.4]$ & $0.19[0.03,0.35]$ & $0.14[-0.07,0.34]$ & $0.33[0.13,0.53]$ \\
\hline Problem type (complex safe zero) & $0.58[0.46,0.71]$ & $0.03[-0.1,0.16]$ & $0.47[0.28 .0 .64]$ & $0[-0.18,0.18]$ \\
\hline Problem type (risky) & $0.09[-0.07,0.24]$ & $0.24[0.08,0.4]$ & $0.06[-0.15,0.27]$ & $0.25[0.04,0.44]$ \\
\hline Age group (older) & $-0.23[-0.48,0.03]$ & $-0.01[-0.26,0.24]$ & $-0.28[-0.59,0.03]$ & $0.03[-0.26,0.34]$ \\
\hline Higher EV choice $=$ higher $\mathrm{CV}$ choice & $2.09[2,2.17]$ & $2.2[2.12,2.28]$ & $2.09[2,2.18]$ & $2.21[2.12,2.3]$ \\
\hline EV difference & $0.01[0.01,0.02]$ & $-0.01[-0.01,0]$ & $0.01[0.01,0.02]$ & $-0.01[-0.01,0]$ \\
\hline Numeracy & $0.11[-0.01,0.22]$ & $-0.04[-0.15,0.06]$ & $0.11[0,0.22]$ & $-0.04[-0.15,0.07]$ \\
\hline Self-reported risk preference & $0.1[0.04,0.15]$ & $-0.01[-0.04,0.07]$ & $0.1[0.04,0.16]$ & $0.01[-0.04,0.06]$ \\
\hline Gender (male) & $0.11[-0.13,0.35]$ & $0.13[-0.12,0.36]$ & $0.1[-0.17,0.36]$ & $0.13[-0.1,0.37]$ \\
\hline Problem Type (simple safe $) \times$ Age Group (older) & & & $-0.28[-0.55,-0.02]$ & $0.06[-0.2,0.31]$ \\
\hline Problem Type (complex safe) $\times$ Age Group (older) & & & $0.22[-0.05,0.47]$ & $-0.28[-0.53,-0.03]$ \\
\hline Problem Type $($ complex safe zero $) \times$ Age Group $($ older $)$ & & & $0.24[-0.01,0.5]$ & $0.07[-0.19,0.31]$ \\
\hline Problem Type (risky) $\times$ Age Group (older) & & & $0.06[-0.21,0.32]$ & $-0.02[-0.26,0.24]$ \\
\hline
\end{tabular}

Note. $\quad \mathrm{EV}=$ expected value; $\mathrm{CV}=$ coefficient of variation. "Higher $\mathrm{EV}$ choice $=$ Higher $\mathrm{CV}$ choice" indicates how the tendency to choose the option with the higher risk $(\mathrm{CV})$ changes when this option also has a higher EV than the alternative option. Boldface indicates credible effects. 


\section{Appendix E}

\section{Testing the Effect of Certainty on the CPT Parameters}

We tested the impact of certainty - the factor highlighted by Mather et al. (2012) - on the CPT parameters. In a series of Bayesian GLMs, we used the CPT parameters $(\rho, \gamma$, and $\alpha)$ as outcome variables. In the main effect models, we used the factors age group and problem type as fixed effects. We specified the complex safe condition as the reference condition for the factor problem type. The effect of problem type (risky) captures the effect of offering two risky options rather than a safe and a risky option, while controlling for complexity differences. To further test whether CPT parameters were more affected in older than in younger adults, we calculated a second set of models that additionally included the interaction between problem type and age group (interaction models). The coefficients for these models are displayed in Table E1 for Study 1 and in Table E2 for Study 2.

First, we evaluated the results for the effect of certainty on response noise ( $\rho$ parameter). In Study 1, there was a credible positive main effect of problem type (risky) in the domain of gains, indicating that response noise was lower in the risky condition than in the complex safe condition. There were no credible main effects of problem type (risky) on the response noise parameter in the domain of losses in Study 1, and in both domains in Study 2. In Study 1, there was a credible negative interaction between problem type (risky) and age group in the domain of gains. This indicates that the decrease in response noise in the risky relative to the complex safe condition was less pronounced in older than in younger adults. The other interactions between problem type (risky) and age group were not credible.

Next, we evaluated differences in probability weighting $(\gamma$ parameter) due to the availability of a safe option. In the main effect models for both domains and in both studies, the credible and positive main effect of problem type (risky) indicates that participants showed more linear probability weighting in the condition

Table E1

Regression Coefficients From the Regressions on Cumulative Prospect Theory Parameters in Study 1 (Reference Condition: Complex Safe; Reference Age Group: Older Adults)

\begin{tabular}{|c|c|c|c|c|}
\hline \multirow[b]{2}{*}{ Predictor } & \multicolumn{2}{|c|}{ Gain } & \multicolumn{2}{|c|}{ Loss } \\
\hline & Main effect model & Interaction model & Main effect model & Interaction model \\
\hline \multicolumn{5}{|c|}{ Outcome variable: $\rho$ (response noise) } \\
\hline (Intercept) & $0.1[0.09,0.11]$ & $0.09[0.07,0.1]$ & $0.18[0.16,0.2]$ & $0.16[0.14,0.19]$ \\
\hline Age group (older) & $-0.03[-0.04,-0.02]$ & $-0.01[-0.02,0.01]$ & $-0.07[-0.09,-0.05]$ & $-0.03[-0.07,0]$ \\
\hline Problem type (risky) & $0.07[0.06,0.08]$ & $0.09[0.07,0.11]$ & $0.01[-0.01,0.04]$ & $0.03[0,0.07]$ \\
\hline Problem Type (risky) × Age Group (older) & & $-0.04[-0.07,-0.02]$ & & $-0.04[-0.09,0.01]$ \\
\hline Problem type (simple safe) & $0.1[0.09,0.11]$ & $0.12[0.1,0.13]$ & $0.09[0.07,0.12]$ & $0.12[0.09,0.016$ \\
\hline Problem Type $($ simple safe $) \times$ Age Group (older) & & $-0.04[-0.06,-.01]$ & & $-0.06[-0.11,-0.01]$ \\
\hline \multicolumn{5}{|c|}{ Outcome variable: $\gamma$ (probability weighting) } \\
\hline (Intercept) & $1.21[1.16,1.26]$ & $1.22[1.16,1.28]$ & $1.17[1.13,1.21]$ & $1.07[1.03,1.12]$ \\
\hline Age group (older) & $-0.06[-0.11,0]$ & $-0.08[-0.17,0.01]$ & $-0.02[-0.07,0.02]$ & $0.17[0.1,0.24]$ \\
\hline Problem type (risky) & $0.18[0.11,0.24]$ & $0.22[0.13,0.31]$ & $0.11[0.05,0.16]$ & $0.23[0.17,0.3]$ \\
\hline Problem Type (risky) × Age Group (older) & & $-0.09[-0.22,0.03]$ & & $-0.26[-0.36,-0.16]$ \\
\hline Problem type (simple safe) & $-0.44[-0.5,-0.37]$ & $-0.51[-0.6,-0.42]$ & $-0.39[-0.44,-0.34]$ & $-0.23[-0.3,-0.16]$ \\
\hline Problem Type (simple safe) $\times$ Age Group (older) & & $0.16[0.03,0.29]$ & & $-0.33[-0.43,-0.23]$ \\
\hline \multicolumn{5}{|c|}{ Outcome variable: $\alpha$ (outcome sensitivity) } \\
\hline (Intercept) & $0.96[0.91,1.01]$ & $0.93[0.87,0.99]$ & $1.11[1.04,1.18]$ & $1.14[1.06,1.21]$ \\
\hline Age group (older) & $-0.1[-0.15,-0.04]$ & $-0.04[-0.13,0.05]$ & $0.02[-0.05,0.08]$ & $-0.03[-0.15,0.08]$ \\
\hline Problem type (risky) & $-0.56[-0.63,-0.5]$ & $-0.61[-0.7,-0.52]$ & $-0.4[-0.48,-0.32]$ & $-0.54[-0.65,-0.44]$ \\
\hline Problem Type (risky) × Age Group (older) & & $0.1[-0.03,0.22]$ & & $0.29[0.13,0.45]$ \\
\hline Problem type (simple safe) & $-0.24[-0.31,-0.17]$ & $-0.12[-0.21,-0.03]$ & $0.06[-0.02,0.14]$ & $0.12[0.01,0.23]$ \\
\hline Problem Type (simple safe) $\times$ Age Group (older) & & $-0.26[-0.38,-0.12]$ & & $-0.13[-0.29,0.02]$ \\
\hline
\end{tabular}

Note. Boldface indicates credible effects. 
Table E2

Regression Coefficients From the Regressions on Cumulative Prospect Theory Parameters in Study 2 (Reference Condition: Complex Safe; Reference Age Group: Older Adults)

\begin{tabular}{|c|c|c|c|c|}
\hline \multirow[b]{2}{*}{ Predictor } & \multicolumn{2}{|c|}{ Gain } & \multicolumn{2}{|c|}{ Loss } \\
\hline & Main effect model & Interaction model & Main effect model & Interaction model \\
\hline \multicolumn{5}{|c|}{ Outcome variable: $\rho$ (response noise) } \\
\hline (Intercept) & $0.17[0.08,0.26]$ & $0.14[0.02,0.26]$ & $0.17[0.12,0.22]$ & $0.11[0.05,0.17]$ \\
\hline Age group (older) & $-0.14[-0.22,-0.06]$ & $-0.08[-0.25,0.1]$ & $-0.15[-0.19,-0.11]$ & $-0.03[-0.12,0.06]$ \\
\hline Problem type (complex safe zero) & $0.51[0.4,0.64]$ & $0.44[0.26,0.61]$ & $0.43[0.36,0.49]$ & $0.4[0.32,0.49]$ \\
\hline Problem Type (complex safe zero) $\times$ Age Group (older) & & $0.16[-0.08,0.41]$ & & $0.04[-0.08,0.17]$ \\
\hline Problem type (risky) & $0.01[-0.12,0.14]$ & $0.01[-0.17,0.18]$ & $0.04[-0.02,0.11]$ & $0.07[-0.02,0.16]$ \\
\hline Problem Type (risky) $\times$ Age Group (older) & & $0.01[-0.24,0.25]$ & & $-0.05[-0.17,0.08]$ \\
\hline Problem type (simple safe zero) & $1.32[1.2,1.45]$ & $1.58[1.4,1.74]$ & $0.72[0.65,0.79]$ & $0.99[0.9,1.08]$ \\
\hline Problem Type (simple safe zero) $\times$ Age Group (older) & & $-0.51[-0.74,-0.26]$ & & $-0.56[-0.68,-0.43]$ \\
\hline Problem type (simple safe) & $0.08[-0.05,0.2]$ & $0.06[-0.11,0.23]$ & $0.07[0,0.14]$ & $0.1[0.01,0.18]$ \\
\hline Problem Type (simple safe) × Age Group (older) & & $0.04[-0.21,0.28]$ & & $-0.04[-0.17,0.08]$ \\
\hline \multicolumn{5}{|c|}{ Outcome variable: $\gamma$ (probability weighting) } \\
\hline (Intercept) & $0.74[0.68,0.79]$ & $0.76[0.69,0.83]$ & $0.87[0.83,0.92]$ & $0.97[0.92,1.03]$ \\
\hline Age group (older) & $0.22[0.18,0.27]$ & $0.18[0.08,0.28]$ & $0.25[0.22,0.29]$ & $0.05[-0.02,0.13]$ \\
\hline Problem type (complex safe zero) & $0.2[0.13,0.28]$ & $0.08[-0.02,0.18]$ & $0.27[0.21,0.33]$ & $-0.05[-0.12,0.02]$ \\
\hline Problem Type (complex safe zero) $\times$ Age Group (older) & & $0.25[0.1,0.38]$ & & $0.63[0.53,0.73]$ \\
\hline Problem type (risky) & $0.4[0.33,0.47]$ & $0.34[0.24,0.44]$ & $0.23[0.17,0.29]$ & $0.18[0.11,0.26]$ \\
\hline Problem Type (risky) $\times$ Age Group (older) & & $0.12[-0.02,0.26]$ & & $0.1[0,0.2]$ \\
\hline Problem type (simple safe zero) & $-0.14[-0.21,-0.07]$ & $-0.1[-0.2,0]$ & $0.02[-0.04,0.08]$ & $-0.18[-0.25,-0.11]$ \\
\hline Problem Type (simple safe zero) $\times$ Age Group (older) & & $-0.08[-0.22,0.06]$ & & $0.4[0.3,0.5]$ \\
\hline Problem type (simple safe) & $-0.13[-0.2,-0.06]$ & $-0.1[-0.2,0]$ & $-0.21[-0.27,-0.15]$ & $-0.15[-0.22,-0.08]$ \\
\hline Problem Type (simple safe) $\times$ Age Group (older) & & $-0.06[-0.2,0.09]$ & & $-0.12[-0.22,-0.02]$ \\
\hline \multicolumn{5}{|c|}{ Outcome variable: $\alpha$ (outcome sensitivity) } \\
\hline (Intercept) & $0.81[0.76,0.86]$ & $0.68[0.62,0.74]$ & $1.04[0.98,1.11]$ & $0.96[0.88,1.04]$ \\
\hline Age group (older) & $-0.01[-0.05,0.03]$ & $0.25[0.17,0.34]$ & $0.1[0.04,0.15]$ & $0.26[0.15,0.37]$ \\
\hline Problem type (complex safe zero) & $0.16[0.09,0.22]$ & $0.25[0.16,0.33]$ & $0.15[0.07,0.23]$ & $0.15[0.04,0.27]$ \\
\hline Problem Type $($ complex safe zero) $\times$ Age Group (older) & & $-0.19[-0.31,-0.06]$ & & $0[-0.16,0.15]$ \\
\hline Problem type (risky) & $-0.55[-0.61,-0.48]$ & $-0.34[-0.31,-0.06]$ & $-0.26[-0.34,-0.17]$ & $-0.01[-0.13,0.11]$ \\
\hline Problem Type (risky) $\times$ Age Group (older) & & $-0.42[-0.55,-0.3]$ & & $-0.49[-0.65,-0.33]$ \\
\hline Problem type (simple safe zero) & $-0.11[-0.18,-0.05]$ & $0.1[0.01,0.18]$ & $0.01[-0.07,0.09]$ & $0.11[0,0.23]$ \\
\hline Problem Type (simple safe zero) $\times$ Age Group (older) & & $-0.42[-0.54,-0.29]$ & & $-0.21[-0.37,-0.04]$ \\
\hline Problem type (simple safe) & $-0.18[-0.24,-0.11]$ & $-0.04[-0.12,0.05]$ & $-0.14[-0.23,-0.05]$ & $-0.08[-0.2,0.03]$ \\
\hline Problem Type (simple safe) $\times$ Age Group (older) & & $-0.28[-0.41,-0.16]$ & & $-0.11[-0.27,0.05]$ \\
\hline
\end{tabular}

Note. Boldface indicates credible effects.

with two risky options than in the condition with a complex safe option. That is, when a safe option was available, probability weighting was less linear, irrespective of the complexity of the safe option. This indicates stronger overweighting of certainty, typically assumed to accommodate the certainty effect. Was this effect more pronounced in older adults? In Study 1, the interaction between age group and problem type (risky) was not credible for the domain of gains, but credible in the domain of losses. This indicates that in the domain of losses the effect of certainty on probability weighting that persists after controlling for complexity may be more pronounced in the younger, not the older, adults. That is, across all participants we find evidence for an effect of certainty on probability weighting beyond the effect of complexity. Nevertheless, the results do not support Mather et al.'s (2012) notion of an increased certainty effect in older adults-only one interaction was credible and it pointed into the opposite direction.
Finally, we evaluated how the availability of a safe option affected outcome sensitivity ( $\alpha$ parameter). The main effect models showed a negative effect of problem type (risky) on outcome sensitivity in both domains and in both studies, indicating that participants' outcome sensitivity parameters were lower when both options were risky than when a complex safe option was available. This effect was less pronounced in older adults in both domains in Study 2 and more pronounced in older adults in the domain of losses in Study 1, indicated by credible interaction terms. Although the availability of a safe outcome seems to affect outcome sensitivity differently in both age groups, there is no consistent evidence as to the direction of this effect. In conclusion, these results suggest that the availability of a safe option affects several aspects of decision making under risk, as reflected by CPT, even after controlling for differences in the complexity of safe and risky options. 


\section{Appendix F}

\section{Analysis of Decision Quality}

We tested whether decision quality-that is, the tendency to choose the option with the higher expected value-was associated with the manipulation of option complexity, using the data from the risky choice task of both Study 1 and Study 2. We conducted Bayesian mixed-effect logistic regressions to predict the choice of the option with the higher expected value, using problem type and age group (main effect model) as well as their interaction (interaction model) as fixed effects. The models further included fixed effects for the expected value difference between options, a dummy variable indicating whether the option with the higher expected value was also riskier, each participant's numeracy score, self-reported risk preference, and gender. The models included a random intercept for each participant. Separate models were calculated for the gain and loss domain, for each study. The coefficients for these models are displayed in Table F1.

We first evaluate the main effect models. Decision quality did not differ between younger and older adults in either domain in Study 1. Older adults' decision quality was credibly lower than younger adults' in the gain domain in Study 2. In both studies, decision quality was higher for participants with higher numeracy scores, and in trials with greater expected value differences between the options. In the domain of gains, participants were less likely to choose the option with the higher expected value if it was also the riskier option (reflecting risk aversion in the domain of gains), in both studies. In the domain of losses, participants were more likely to choose the option with the higher expected value if it was also the riskier option (reflecting risk seeking in the domain of losses), in both studies. There were no credible main effects of gender on decision quality, except in the domain of gains in Study 2. Decision quality decreased when the complexity of the safe option increased, as indicated by credible negative main effects of problem type (complex safe) in both domains and in both studies. The interaction models further show that the effect of problem type (complex safe) on decision quality was equally pronounced in both age groups in both studies, as indicated by the interaction effect between problem type (complex safe) and age group (older) not being credible.

How did the presence of zero outcomes in Study 2 affect decision quality? The GLMER analyses show that problem type (simple safe zero) and problem type (complex safe zero) had positive main effects on the tendency to choose the higher EV option in the domain of gains, and did not credibly affect the tendency to choose the higher EV option in the domain of losses. There were credible and positive interactions between problem type (simple safe zero) and age group (older), as well as between problem type (complex safe zero) and age group (older). These interactions indicate that decision quality improved more strongly in older than in younger adults when zero outcomes were available. 
Table F1

Regression Coefficients and 95\% Posterior Intervals From the Mixed-Effects Logistic Regression of Responses of Decision Quality (Measured as the Tendency to Choose the Option With the Higher Expected Value) in the Risky Choice Task of Studies 1 and 2, by Domain

\begin{tabular}{cccccc}
\hline & & \multicolumn{2}{c}{ Main effect model } & \\
\cline { 3 - 3 } & Predictor & Gain & Loss & Gain \\
\hline
\end{tabular}

Outcome variable: Choice of option with higher EV

Study 1

(Intercept)

Problem type (complex safe)

Problem type (risky)

Age group (older)

Higher EV choice $=$ higher $\mathrm{CV}$ choice

EV difference

Numeracy

Self-reported risk preference

Gender (male)

Problem Type (complex safe) $\times$ Age Group (older)

Problem Type (risky) $\times$ Age Group (older)

Study 2

(Intercept)

Problem type (simple safe zero)

Problem type (complex safe)

Problem type (complex safe zero)

Problem type (risky)

Age group (older)

Higher EV choice $=$ higher $\mathrm{CV}$ choice

EV difference

Numeracy

Self-reported risk preference

Gender (male)

Problem Type (simple safe zero) $\times$ Age Group (older)

Problem Type (complex safe) $\times$ Age Group (older)

Problem Type (complex safe zero) $\times$ Age Group (older)

Problem Type (risky) $\times$ Age Group (older)

$$
\begin{array}{ccc}
-0.04[-0.56,0.44] & -0.17[-0.67,0.36] & -0.04[-0.56,0.46] \\
\mathbf{- 0 . 5 5}[-\mathbf{0 . 7}, \mathbf{- 0 . 4}] & \mathbf{- 0 . 6 2}[\mathbf{- 0 . 7 8 , - 0 . 4 7}] & \mathbf{- 0 . 5 9}[-\mathbf{0 . 8 1}, \mathbf{- 0 . 3 7}] \\
0.16[0,0.31] & -0.08[-0.25,0.08] & 0.14[-0.08,0.37] \\
-0.15[-0.4,0.09] & -0.2[-0.45,0.05] & -0.18[-0.49,0.12] \\
\mathbf{- 1 . 1 3}[-\mathbf{1 . 2 6}, \mathbf{- 1}] & \mathbf{0 . 3 8}[\mathbf{0 . 2 5}, \mathbf{0 . 5}] & \mathbf{- 1 . 1 3}[-\mathbf{1 . 2 6}, \mathbf{- 1}] \\
\mathbf{0 . 0 8}[\mathbf{0 . 0 7 , 0 . 0 8}] & \mathbf{0 . 0 6}[\mathbf{0 . 0 5}, \mathbf{0 . 0 6}] & \mathbf{0 . 0 8}[\mathbf{0 . 0 7}, \mathbf{0 . 0 8}] \\
\mathbf{0 . 3 4}[\mathbf{0 . 2 3}, \mathbf{0 . 4 5}] & \mathbf{0 . 3 7}[\mathbf{0 . 2 6}, \mathbf{0 . 4 8}] & \mathbf{0 . 3 4}[\mathbf{0 . 2 4} \mathbf{0 . 4 5}] \\
-0.01[-0.06,0.05] & -0.02[-0.07,0.04] & -0.01[-0.06,0.05] \\
0.23[-0.02,0.5] & 0.22[-0.03,0.48] & 0.23[-0.03,0.49] \\
& & 0.07[-0.22,0.36] \\
& & 0.02[-0.29,0.32]
\end{array}
$$

$\mathbf{0 . 4 9}[\mathbf{0 . 1 6}, \mathbf{0 . 8 1}]$
$\mathbf{0 . 1 9}[\mathbf{0 . 0 3 , 0 . 3 5}]$
$-\mathbf{0 . 1 9}[-\mathbf{0 . 3 4},-\mathbf{0 . 0 6}]$
$\mathbf{0 . 3 5}[\mathbf{0 . 1 8}, \mathbf{0 . 5}]$
$0.11[-0.04,0.26]$
$-\mathbf{0 . 3 2}[-\mathbf{0 . 5 1},-\mathbf{0 . 1 4}]$
$-\mathbf{1 . 3 1}[-\mathbf{1 . 4},-\mathbf{1 . 2 2}]$
$\mathbf{0 . 0 6}[\mathbf{0 . 0 5}, \mathbf{0 . 0 6}]$
$\mathbf{0 . 1 4}[\mathbf{0 . 0 6 , 0 . 2 2}]$
$0.04[0,0.08]$
$\mathbf{0 . 2 2}[\mathbf{0 . 0 3}, \mathbf{0 . 4}]$

$0.11[-0.2,0.42]$

$0.07[-0.09,0.23]$

$-0.39[-0.53,-0.25]$

$0.05[-0.1,0.22]$

$0.07[-0.08,0.22]$

$-0.17[-0.34,0.01]$

$0.31[0.23,0.39]$

$0.06[0.05,0.06]$

$0.14[0.07,0.22]$

$0[-0.04,0.03]$

$0.01[-0.16,0.18]$
0.67 [0.31, 1.01]

$-0.12[-0.33,0.09]$

$-0.18[-0.4,0.03]$

$-0.02[-0.23,0.19]$

$0.04[-0.18,0.26]$

$-0.67[-0.93,-0.29]$

-1.31 [-1.4, -1.23]

$0.06[0.05,0.06]$

$0.14[0.06,0.22]$

$0.04[0,0.08]$

$0.22[0.04,0.4]$

$0.61[0.35,0.88]$

$-0.02[-0.3,0.26]$

$0.7[0.44,0.96]$

$0.13[-0.15,0.42]$
$-0.12[-0.62,0.39]$

$\mathbf{- 0 . 6 5}[-0.87,-0.42]$

$-0.22[-0.46,0.01]$

$-0.31[-0.62,0.02]$

$0.38[0.24,0.51]$

$0.06[0.05,0.06]$

0.37 [0.26, 0.48]

$-0.02[-0.07,0.04]$

$0.22[-0.04,0.47]$

$0.05[-0.25,0.36]$

0.27 [-0.05. 0.59]

$0.31[-0.02,0.64]$

$-0.28[-0.5,-0.06]$

$-0.44[-0.66,-0.22]$

$-0.29[-0.52,-0.08]$

$0.01[-0.22,0.24]$

$-0.54[-0.82,-0.28]$

$0.31[0.23,0.39]$

$0.06[0.05,0.06]$

$0.15[0.07,0.22]$

$0[-0.04,0.03]$

$0.01[-0.15,0.18]$

$0.67[0.39,0.93]$

$0.08[-0.21,0.36]$

$0.67[0.4,0.93]$

$0.11[-0.2,0.4]$

Note. $\quad \mathrm{EV}=$ expected value; $\mathrm{CV}=$ coefficient of variation. "Higher $\mathrm{EV}$ choice $=$ higher $\mathrm{CV}$ choice" indicates how the tendency to choose the option with the higher EV changes when this option also has higher risk (CV) than the alternative option. Boldface indicates credible effects.

Received November 6, 2018

Revision received December 27, 2019

Accepted December 30, 2019 